\title{
Left-Invariant Diffusions on the Space of Positions and Orientations and their Application to Crossing-Preserving Smoothing of HARDI images
}

\author{
Remco Duits • Erik Franken
}

Received: 15 May 2009 / Accepted: 15 March 2010 / Published online: 31 March 2010

(c) The Author(s) 2010. This article is published with open access at Springerlink.com

\begin{abstract}
HARDI (High Angular Resolution Diffusion Imaging) is a recent magnetic resonance imaging (MRI) technique for imaging water diffusion processes in fibrous tissues such as brain white matter and muscles. In this article we study left-invariant diffusion on the group of 3D rigid body movements (i.e. 3D Euclidean motion group) $S E(3)$ and its application to crossing-preserving smoothing of HARDI images. The linear left-invariant (convection-)diffusions are forward Kolmogorov equations of Brownian motions on the space of positions and orientations in $3 \mathrm{D}$ embedded in $S E(3)$ and can be solved by $\mathbb{R}^{3} \rtimes S^{2}$-convolution with the corresponding Green's functions. We provide analytic approximation formulas and explicit sharp Gaussian estimates for these Green's functions. In our design and analysis for appropriate (nonlinear) convection-diffusions on HARDI data we explain the underlying differential geometry on $S E(3)$. We write our leftinvariant diffusions in covariant derivatives on $S E(3)$ using the Cartan connection. This Cartan connection has constant curvature and constant torsion, and so have the exponential curves which are the auto-parallels along which our leftinvariant diffusion takes place. We provide experiments of
\end{abstract}

\footnotetext{
R. Duits $(\bowtie)$

Department of Mathematics and Computer Science, CASA

Applied Analysis, Eindhoven University of Technology,

P.O. Box 513, 5600 MB Eindhoven, The Netherlands

e-mail: R.Duits@tue.nl

R. Duits · E. Franken

Department of Biomedical Engineering, BMIA Biomedical Image Analysis, Eindhoven University of Technology,

P.O. Box 513, 5600 MB Eindhoven, The Netherlands

E. Franken

e-mail: E.M.Franken@tue.nl
}

our crossing-preserving Euclidean-invariant diffusions on artificial HARDI data containing crossing-fibers.

Keywords High angular resolution diffusion imaging (HARDI) · Scale spaces · Lie groups · Partial differential equations

\section{Introduction}

High Angular Resolution Diffusion Imaging (HARDI) is a recent magnetic resonance imaging technique for imaging water diffusion processes in fibrous tissues such as brain white matter and muscles. HARDI provides for each position in 3-space (i.e. $\mathbb{R}^{3}$ ) and for each orientation (antipodal pairs on the 2-sphere $S^{2}$ ) an MRI signal attenuation profile, which can be related to the local diffusivity of water molecules in the corresponding direction. It is generally believed that such profiles provide rich information in fibrous tissues. DTI (Diffusion Tensor Imaging) is a related technique, producing a positive symmetric rank-2 tensor field. A DTI tensor (at each position in 3-space) can also be related to a distribution on the 2-sphere, albeit with limited angular resolution. DTI is incapable of representing areas with complex multimodal diffusivity profiles, such as induced by crossing, "kissing", or bifurcating fibres. HARDI, on the other hand, does not suffer from this problem, because it is not restricted to functions on the 2-sphere induced by a quadratic form, see Fig. 1 where we used glyph visualizations as defined in Definition 1. For the purpose of tractography (detection of biological fibers) and visualization, DTI and HARDI data should be enhanced such that fiber junctions are maintained, while reducing high frequency noise and small incoherent structures in the joined domain 


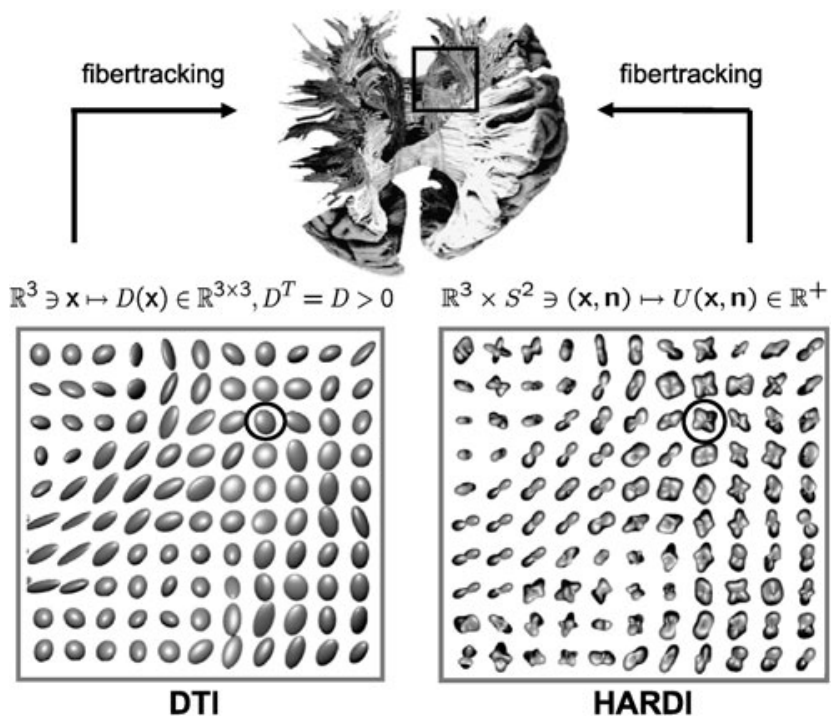

Fig. 1 This figure shows glyph visualizations of HARDI and DTI-images of a 2D slice in the brain where neural fibers in the corona radiata cross with neural fibers in the corpus callosum. In HARDI and DTI socalled "glyphs" (i.e. angular diffusivity profiles) reflect, per position, the local diffusivity of water in all directions. More, precisely, to a DTI tensor field $\mathbf{x} \mapsto D(\mathbf{x})$ we associate a distribution on positions and orientations $(\mathbf{x}, \mathbf{n}) \mapsto \mathbf{n}^{T} D(\mathbf{x}) \mathbf{n}$ which is depicted on the left. The rank-2 limitation of a DTI tensor constrains the corresponding glyph to be ellipsoidal, whereas no such constraint applies to HARDI

of positions and orientations. This crossing-preserving enhancement/diffusion along fibers within distributions defined on the joined space of positions and orientations (such as HARDI and DTI images) is the main objective of this article.

Definition 1 A glyph of a distribution $U: \mathbb{R}^{3} \times S^{2} \rightarrow \mathbb{R}^{+}$ on positions and orientations is a surface $\mathcal{S}_{\mu}(U)(\mathbf{x})=$ $\left\{\mathbf{x}+\mu U(\mathbf{x}, \mathbf{n}) \mathbf{n} \mid \mathbf{n} \in S^{2}\right\} \subset \mathbb{R}^{3}$ for some $\mathbf{x} \in \mathbb{R}^{3}$ and $\mu>0$. A glyph visualization of the distribution $U: \mathbb{R}^{3} \times S^{2} \rightarrow \mathbb{R}^{+}$ is a visualization of a field $\mathbf{x} \mapsto \mathcal{S}_{\mu}(U)(\mathbf{x})$ of glyphs, where $\mu>0$ is a suitable constant.

Promising research has been done on constructing diffusion (or similar regularization) processes on the 2-sphere defined at each spatial locus separately (Descoteaux et al. 2007; Florack and Balmachnova 2008; Florack 2008; Hess et al. 2006) as an essential pre-processing step for robust fiber tracking. In these approaches position and orientation space are decoupled, and diffusion is only performed over the angular part, disregarding spatial context. Consequently, these methods are inadequate for spatial denoising and enhancement, and tend to fail precisely at the interesting locations where fibres cross or bifurcate.

Therefore in this article we extend our recent work on enhancement of elongated structures in 2D greyscale images (van Almsick 2005; Franken and Duits 2009; Franken 2008; original 2D image
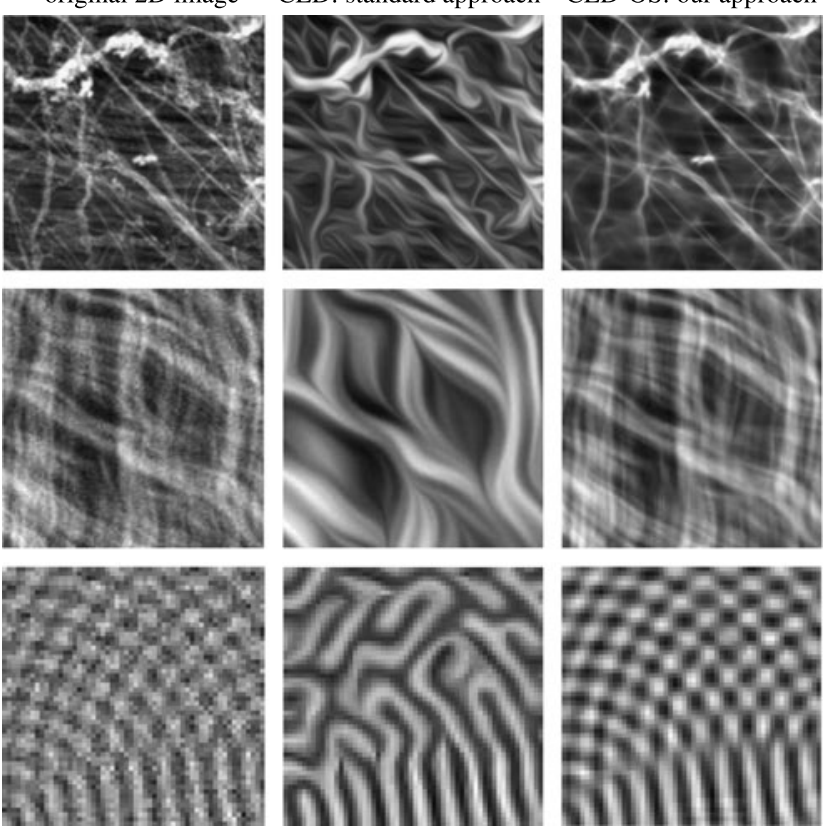

Fig. 2 Left-invariant diffusion on $S E(2)=\mathbb{R}^{2} \rtimes S^{1}$ is the right approach to generically deal with crossings and bifurcations in practice. Left column: original images. Middle column: result of standard coherence enhancing diffusion applied directly in the image domain $\mathbb{R}^{2}$ (CED), cf. (Weickert 1999). Right column: coherence enhancing diffusion via the corresponding invertible orientation score (CED-OS) in the 2D Euclidean motion group SE(2), cf. (Duits and Franken 2010; Franken and Duits 2009). Top row: 2-photon microscopy image of bone tissue. Second row: collagen fibers of the heart. Third row: artificial noisy interference pattern. CED-OS of 2D grey value images is capable of handling crossings and bifurcations, whereas CED produces spurious artifacts at such junctions. In the $3 \mathrm{D}$ case of HARDI images $U: \mathbb{R}^{3} \rtimes S^{2} \rightarrow \mathbb{R}$, we do not have to bother about invertibility of the transform between a grey-value image and its orientation score as the input-data itself already gives rise to a function on the $3 \mathrm{D}$ Euclidean motion group $S E(3)$. This is now simply achieved by setting $\tilde{U}(\mathbf{x}, R)=U\left(\mathbf{x}, R \mathbf{e}_{z}\right), R \in S O(3), \mathbf{x} \in \mathbb{R}^{3}, \mathbf{e}_{z}=(0,0,1)^{T}$ and the challenge rises to generalize our previous work on crossing preserving diffusion to $3 \mathrm{D}$ and to apply the left-invariant diffusion directly on HARDI images

Duits and van Almsick 2008; Duits et al. 2007; Duits and Franken 2009; Duits and Burgeth 2007; Duits and Franken 2010) to the genuinely 3D case of HARDI/DTI, since this approach has proven to be capable of handling all aforementioned problems in various feasibility studies, see Fig. 2. In contrast to the previous works on diffusion of DTI/HARDI images (Descoteaux et al. 2007; Florack and Balmachnova 2008; Florack 2008; Hess et al. 2006; Özarslan and Mareci 2003), we consider both the spatial and the orientational part to be included in the domain, so a HARDI dataset is considered as a function $U: \mathbb{R}^{3} \times S^{2} \rightarrow \mathbb{R}$. Furthermore, we explicitly employ the proper underlying group structure, that naturally arises by embedding the coupled space of positions and orientations into the group $S E(3)$ of $3 \mathrm{D}$ rigid motions. The relevance of group theory in DTI/HARDI imag- 


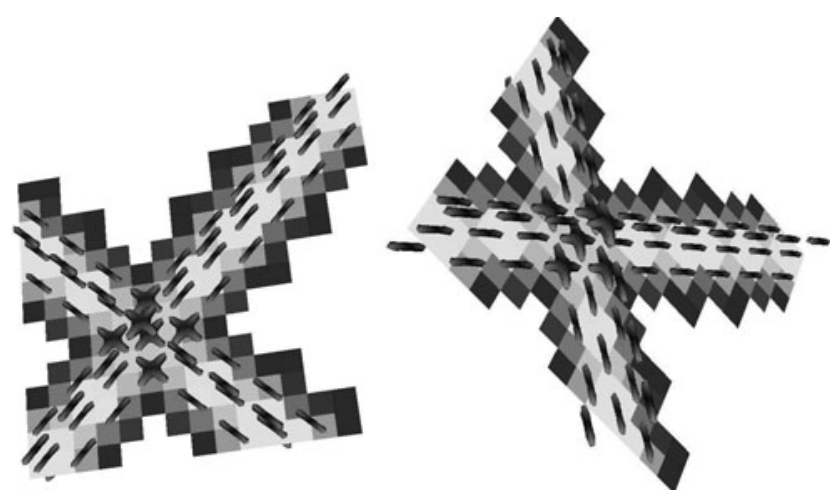

Fig. 3 Visualization of a simple HARDI image $(x, y, z, \mathbf{n}(\beta, \gamma))$ $\mapsto U(x, y, z, \mathbf{n}(\beta, \gamma))$ containing two crossing straight lines, visualized using Q-ball glyphs in the DTI tool from two different viewpoints. At each spatial position $\mathbf{x}$ a glyph (cf. Fig. 1 and Definition 1) is displayed

ing has also been stressed in promising and well-founded recent works (Gur and Sochen 2009; Gur and Sochen 2005; Fletcher and Joshi 2007). However, these works rely on biinvariant Riemannian metrics on compact groups (such as $S O(3)$ ) and in our case the group $S E(3)$ is neither compact nor does it permit a bi-invariant metric (Arsigny et al. 2006; Duits and Franken 2010, Part II). In general the advantage of our approach on $S E(3)$ is that we can enhance the original HARDI/DTI data using simultaneously orientational and spatial neighborhood information, which potentially leads to improved enhancement and detection algorithms. Figure 3 shows an example clarifying the structure of a HARDI image.

This paper is organized as follows. In Sect. 2 we will start with the introduction of the group structure on the domain of a HARDI image. Here we will explain that the domain of a HARDI image of positions and orientations carries a semi-direct product structure rather than a direct Cartesian product structure reflecting a natural coupling between position and orientation. We embed the space of positions and orientations into the group of positions and rotations in $\mathbb{R}^{3}$, which is commonly denoted by $S E(3)=\mathbb{R}^{3} \rtimes S O(3)$. As a result we must write

$\mathbb{R}^{3} \rtimes S^{2}:=\mathbb{R}^{3} \rtimes S O(3) /(\{\mathbf{0}\} \times S O(2))$

rather than $\mathbb{R}^{3} \times S^{2}$ for the domain of a HARDI image.

In Sect. 3 we will discuss basic tools from group theory, which serve as key ingredients in our diffusions on HARDI images later on. Within this section we also provide an example to embed a recent paper (Barmpoutis et al. 2008) by Barmpoutis et al. on smoothing of DTI/HARDI data in our group theoretical framework. We show that their kernel operator indeed is a correct left-invariant group convolution on $\mathbb{R}^{3} \rtimes S^{2}$. However, their kernel does not satisfy the semi- group property and does not relate to diffusion or Tikhonov energy minimization on $\mathbb{R}^{3} \rtimes S^{2}$.

Subsequently, in Sect. 4 we will derive all linear left-invariant convection-diffusion equations on $S E(3)$ and $\mathbb{R}^{3} \rtimes S^{2}$ (the actual domain of HARDI images) and show that the solutions of these convection-diffusion equations are given by group-convolution with the corresponding Green's functions, which we explicitly approximate later. Furthermore, in Sect. 4.2, we put an explicit connection with probability theory and random walks in the space of orientations and positions. This connection is established by the fact that the convection-diffusion equations are Fokker-Planck (i.e. forward Kolmogorov) equations of stochastic processes (random walks) on the space of orientations and positions. This in turn brings a connection to the actual measurements of water-molecules in oriented fibrous tissues. Symmetry requirements for the linear diffusions on $\mathbb{R}^{3} \rtimes S^{2}$ yields the following cases:

1. The natural 3D generalizations of Mumford's direction process on $\mathbb{R}^{2} \rtimes S^{1}$ (Mumford 1994; Duits and van Almsick 2008), which is a contour completion process in the group $S E(2)=\mathbb{R}^{2} \rtimes S^{1} \equiv \mathbb{R}^{2} \rtimes S O(2)$ of $2 \mathrm{D}$-positions and orientations;

2. The natural 3D generalizations of a (horizontal) random walk on $\mathbb{R}^{2} \rtimes S^{1}$, cf. (Duits and Franken 2010), corresponding to the diffusions proposed by Citti and Sarti (2006), which is a contour enhancement process in the group $S E(2)=\mathbb{R}^{2} \rtimes S^{1} \equiv \mathbb{R}^{2} \rtimes S O(2)$ of $2 \mathrm{D}$-positions and orientations;

3. Gaussian scale space (Iijima 1962; Koenderink 1984; Alvarez et al. 1993; Duits et al. 2004) over position space, i.e. spatial linear diffusion;

4. Gaussian scale space over angular space (2-sphere) (Descoteaux et al. 2007; Özarslan and Mareci 2003; Florack and Balmachnova 2008; Florack 2008; Hess et al. 2006), i.e. angular linear diffusion,

or combinations of these four types of convection-diffusions. Previous approaches of HARDI-diffusions (Descoteaux et al. 2007; Özarslan and Mareci 2003; Florack and Balmachnova 2008) fit in our framework (third and fourth item), but it is rather the first two cases that are challenging as they involve a natural coupling between position and orientation space and thereby allow appropriate treatment of crossing fibers. In Sect. 5 we will explore the underlying differential geometry of our diffusions on HARDI-orientation scores. By means of the Cartan connection on $S E(3)$ we put a useful relation to rigid body mechanics expressed in moving frames of reference, providing geometrical intuition behind our left-invariant (convection-)diffusions on HARDI data. Furthermore, we show that our (convection-)diffusion may be expressed in covariant derivatives and we show that both convection and diffusion locally take place along the 
exponential curves in $S E(3)$, that are explicitly derived in Sect. 5.1. In Sect. 6 we will derive suitable formulas and Gaussian estimates for the Green's functions of linear leftinvariant convection-diffusions on HARDI images. These formulas are used in the subsequent section in our numerical convolution-schemes solving the left-invariant diffusions on HARDI images.

Section 7 explains the basic numerics of our left-invariant PDE- and/or convolution schemes, which we use in the subsequent experimental section. Section 8 contains preliminary results of linear left-invariant diffusion on artificial HARDI datasets over the joined coupled domain of positions and orientations (i.e. over $\mathbb{R}^{3} \rtimes S^{2}$ ).

The final section of this paper provides the theory for nonlinear adaptive diffusion on HARDI images, which is a generalization of our nonlinear adaptive diffusion schemes on the 2D Euclidean motion group (Franken and Duits 2009; Duits and Franken 2010).

\section{The Group Structure on the Domain of a HARDI Image: The Embedding of $\mathbb{R}^{3} \times S^{2}$ into $S E(3)$}

In order to generalize our previous work on line/contourenhancement via left-invariant diffusions on invertible orientation scores of 2D-images we first investigate the group structure on the domain of a HARDI image. Just like orientation scores are scalar-valued functions on the space of 2D-positions and orientations, i.e. the 2D-Euclidean motion group, HARDI images are scalar-valued functions on the space of 3D-positions and orientations. This generalization involves some technicalities since the 2 -sphere $S^{2}=\{\mathbf{x} \in$ $\left.\mathbb{R}^{3} \mid\|\mathbf{x}\|=1\right\}$ is not a Lie-group proper ${ }^{1}$ in contrast to the 1-sphere $S^{1}=\left\{\mathbf{x} \in \mathbb{R}^{2} \mid\|\mathbf{x}\|=1\right\}$. To overcome this problem we embed $\mathbb{R}^{3} \times S^{2}$ into $S E(3)$ which is the group of $3 \mathrm{D}$-rotations and translations (i.e. the group of $3 \mathrm{D}$ rigid motions). As a concatenation of two rigid body movements is again a rigid body movement, the product on $S E(3)$ is given by

$$
\begin{aligned}
& (\mathbf{x}, R)\left(\mathbf{x}^{\prime}, R^{\prime}\right)=\left(R \mathbf{x}^{\prime}+\mathbf{x}, R R^{\prime}\right), \\
& R, R^{\prime} \in S O(3), \quad \mathbf{x}, \mathbf{x}^{\prime} \in \mathbb{R}^{3}
\end{aligned}
$$

The group $S E(3)$ is a semi-direct product of the translation group $\mathbb{R}^{3}$ and the rotation group $S O(3)$, since it uses an isomorphism $R \mapsto(\mathbf{x} \mapsto R \mathbf{x})$ from the rotation group onto the automorphisms on $\mathbb{R}^{3}$. Therefore we write $\mathbb{R}^{3} \rtimes S O(3)$

\footnotetext{
${ }^{1}$ If $S^{2}$ were a Lie-group then its left-invariant vector fields would be non-zero everywhere, contradicting Poincaré's "hairy ball theorem" (proven by Brouwer in 1912), or more generally the Poincaré-Hopf theorem (the Euler-characteristic of an even dimensional sphere $S^{2 n}$ is 2).
}

rather than $\mathbb{R}^{3} \times S O(3)$ which would yield a direct product. The groups $S E(3)$ and $S O(3)$ are not commutative. Throughout this article we will use Euler-angle parametrization for $S O(3)$, i.e. we write a rotation as a product of a rotation around the $z$-axis, a rotation around the $y$-axis and a rotation around the $z$-axis again.

$R=R_{\mathbf{e}_{z}, \gamma} R_{\mathbf{e}_{y}, \beta} R_{\mathbf{e}_{z}, \alpha}$

where all rotations are counter-clockwise, where all rotations are counter-clockwise, i.e.:

$R_{\mathbf{e}_{z}, \gamma}=\left(\begin{array}{ccc}\cos \gamma & -\sin \gamma & 0 \\ \sin \gamma & \cos \gamma & 0 \\ 0 & 0 & 1\end{array}\right)$ and
$R_{\mathbf{e}_{y}, \beta}=\left(\begin{array}{ccc}\cos \beta & 0 & \sin \beta \\ 0 & 1 & 0 \\ -\sin \beta & 0 & \cos \beta\end{array}\right)$

The advantage of the Euler angle parametrization is that it directly parameterizes $S O(3) / S O(2) \equiv S^{2}$ as well. Here we recall that $S O(3) / S O(2)$ denotes the partition of all left cosets which are equivalence classes $[g]=\{h \in S O(3) \mid$ $h \sim g\}=g S O(2)$ under the equivalence relation $g_{1} \sim g_{2} \Leftrightarrow$ $g_{1}^{-1} g_{2} \in S O(2)$ where we identified $S O(2)$ with rotations around the $z$-axis and we have

$$
\begin{aligned}
& S O(3) / S O(2) \ni\left[R_{\mathbf{e}_{z}, \gamma} R_{\mathbf{e}_{y}, \beta}\right] \\
&=\left\{R_{\mathbf{e}_{z}, \gamma} R_{\mathbf{e}_{y}, \beta} R_{\mathbf{e}_{z}, \alpha} \mid \alpha \in[0,2 \pi)\right\} \\
& \leftrightarrow \mathbf{n}(\beta, \gamma):=(\cos \gamma \sin \beta, \sin \gamma \sin \beta, \cos \beta)^{T} \\
&=R_{\mathbf{e}_{z}, \gamma} R_{\mathbf{e}_{y}, \beta} R_{\mathbf{e}_{z}, \alpha} \mathbf{e}_{z} \in S^{2}
\end{aligned}
$$

Like all parameterizations of $S O(3) / S O(2)$, the Euler angle parametrization suffers from the problem that there does not exists a global diffeomorphism from a sphere to a plane. In the Euler-angle parametrization the ambiguity arises at the north and south-pole:

$$
\begin{aligned}
& R_{\mathbf{e}_{z}, \gamma} R_{\mathbf{e}_{y}, \beta=0} R_{\mathbf{e}_{z}, \alpha}=R_{\mathbf{e}_{z}, \gamma-\delta} R_{\mathbf{e}_{y}, \beta=0} R_{\mathbf{e}_{z}, \alpha+\delta}, \quad \text { and } \\
& R_{\mathbf{e}_{z}, \gamma} R_{\mathbf{e}_{y}, \beta=\pi} R_{\mathbf{e}_{z}, \alpha}=R_{\mathbf{e}_{z}, \gamma+\delta} R_{\mathbf{e}_{y}, \beta=\pi} R_{\mathbf{e}_{z}, \alpha+\delta}, \\
& \quad \text { for all } \delta \in[0,2 \pi)
\end{aligned}
$$

Consequently, we occasionally need a second chart to cover $S O(3)$;

$R=R_{\mathbf{e}_{x}, \tilde{\gamma}} R_{\mathbf{e}_{y}, \tilde{\beta}} R_{\mathbf{e}_{z}, \tilde{\alpha}}$,

which again parameterizes $S O(3) / S O(2) \equiv S^{2}$ using different spherical coordinates $\tilde{\beta} \in[-\pi, \pi), \tilde{\gamma} \in\left(-\frac{\pi}{2}, \frac{\pi}{2}\right)$,

$$
\begin{aligned}
& \tilde{\mathbf{n}}(\tilde{\beta}, \tilde{\gamma})=R_{\mathbf{e}_{x}, \tilde{\gamma}} R_{\mathbf{e}_{y}, \tilde{\beta}} \mathbf{e}_{z} \\
& \quad=(\sin \tilde{\beta},-\cos \tilde{\beta} \sin \tilde{\gamma}, \cos \tilde{\beta} \cos \tilde{\gamma})^{T}
\end{aligned}
$$




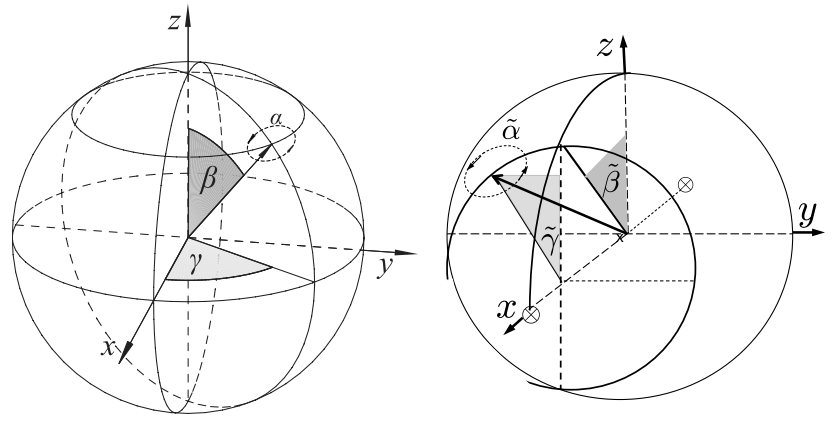

Fig. 4 The two charts which together appropriately parameterize the sphere $S^{2} \equiv S O(3) / S O(2)$ where the rotation-parameters $\alpha$ and $\tilde{\alpha}$ are free. The first chart (left-image) is the common Euler-angle parametrization (1), the second chart is given by (4). The first chart has singularities at north and south-pole (inducing ill-defined parametrization of the left-invariant vector fields (24) at the unity element) whereas the second chart has singularities at $( \pm 1,0,0)$

but which has ambiguities at the intersection of the equator with the $x$-axis

$$
R_{\mathbf{e}_{x}, \tilde{\gamma}} R_{\mathbf{e}_{y}, \tilde{\beta}= \pm \frac{\pi}{2}} R_{\mathbf{e}_{z}, \tilde{\alpha}}=R_{\mathbf{e}_{x}, \tilde{\gamma}-\delta} R_{\mathbf{e}_{y}, \tilde{\beta}= \pm \frac{\pi}{2}} R_{\mathbf{e}_{z}, \tilde{\alpha} \pm \delta},
$$

for all $\delta \in[0,2 \pi)$.

See Fig. 4. Away from the intersection of the $z$ - and $x$-axis with the sphere one can accomplish conversion between the two charts by solving for either $(\tilde{\alpha}, \tilde{\beta}, \tilde{\gamma})$ or $(\alpha, \beta, \gamma)$ in $R_{\mathbf{e}_{x}, \tilde{\gamma}} R_{\mathbf{e}_{y}, \tilde{\beta}} R_{\mathbf{e}_{z}, \tilde{\alpha}}=R_{\mathbf{e}_{z}, \gamma} R_{\mathbf{e}_{y}, \beta} R_{\mathbf{e}_{z}, \alpha}$.

Now that we have explained the isomorphism $\mathbf{n}=R \mathbf{e}_{z} \in$ $S^{2} \leftrightarrow S O(3) / S O(2) \ni[R]$ explicitly in charts, we return to the domain of HARDI images. Considered as a set this domain equals the space of 3D-positions and orientations $\mathbb{R}^{3} \times S^{2}$. However, in order to stress the fundamental embedding of the HARDI domain in $S E(3)$ and the thereby induced (quotient) group-structure we write $\mathbb{R}^{3} \rtimes S^{2}$, which equals the following Lie-group quotient:

$\mathbb{R}^{3} \rtimes S^{2}:=\left(\mathbb{R}^{3} \rtimes S O(3)\right) /(\{\mathbf{0}\} \times S O(2))$.

Here the equivalence relation on the group of rigid-motions $S E(3)=\mathbb{R}^{3} \rtimes S O(3)$ equals

$(\mathbf{x}, R) \sim\left(\mathbf{x}^{\prime}, R^{\prime}\right) \quad \Leftrightarrow \quad \mathbf{x}=\mathbf{x}^{\prime} \quad$ and

$R^{-1} R^{\prime}$ is a rotation around the $z$-axis

and the set of equivalence classes within $S E(3)$ under this equivalence relation (i.e. left cosets) equals the space of coupled orientations and positions and is denoted by $\mathbb{R}^{3} \rtimes S^{2}$.

\section{Tools from Group Theory}

In this article we will consider convection-diffusion operators on the space of HARDI images. We shall model the space of HARDI images by the space of quadratic integrable functions on the coupled space of positions and orientations, i.e. $\mathbb{L}_{2}\left(\mathbb{R}^{3} \rtimes S^{2}\right)$. We will first show that such operators should be left-invariant with respect to the left-action of $S E(3)$ onto the space of HARDI images. This left-action of $S E(3)$ onto $\mathbb{R}^{3} \rtimes S^{2}$ is given by

$g \cdot(\mathbf{y}, \mathbf{n})=(R \mathbf{y}+\mathbf{x}, R \mathbf{n})$,

$g=(\mathbf{x}, R) \in \operatorname{SE}(3), \quad \mathbf{x}, \mathbf{y} \in \mathbb{R}^{3}, \mathbf{n} \in S^{2}, R \in S O(3)$

and it induces the so-called left-regular action of the same group on the space of HARDI images similar to the leftregular action on 3D images (for example orientationmarginals of HARDI images):

Definition 2 The left-regular actions of $S E(3)$ onto $\mathbb{L}_{2}\left(\mathbb{R}^{3} \rtimes S^{2}\right)$ respectively $\mathbb{L}_{2}\left(\mathbb{R}^{3}\right)$ are given by

$$
\begin{gathered}
\left(\mathfrak{L}_{g=(\mathbf{x}, R)} U\right)(\mathbf{y}, \mathbf{n})=U\left(g^{-1} \cdot(\mathbf{y}, \mathbf{n})\right) \\
=U\left(R^{-1}(\mathbf{y}-\mathbf{x}), R^{-1} \mathbf{n}\right), \\
\mathbf{x}, \mathbf{y} \in \mathbb{R}^{3}, \mathbf{n} \in S^{2}, U \in \mathbb{L}_{2}\left(\mathbb{R}^{3} \rtimes S^{2}\right), \\
\left(\mathfrak{U}_{g=(\mathbf{x}, R)} f\right)(\mathbf{y})=f\left(R^{-1}(\mathbf{y}-\mathbf{x})\right), \\
R \in \operatorname{SO}(3), \mathbf{x}, \mathbf{y} \in \mathbb{R}^{3}, f \in \mathbb{L}_{2}\left(\mathbb{R}^{3}\right) .
\end{gathered}
$$

Intuitively, $\mathfrak{U}_{g=(\mathbf{x}, R)}$ represents a rigid motion operator on images, whereas $\mathfrak{L}_{g=(\mathbf{x}, R)}$ represents a rigid motion on HARDI images.

In order to explain the importance of left-invariance of processing HARDI images in general we need to define the following operator.

Definition 3 We define the operator $\mathcal{M}$ which maps a HARDI image $U: \mathbb{R}^{3} \rtimes S^{2} \rightarrow \mathbb{R}^{+}$to its orientation marginal $\mathcal{M} U: \mathbb{R}^{3} \rightarrow \mathbb{R}^{+}$as follows (where $\sigma$ denotes the usual surface measure on $S^{2}$ ):

$$
(\mathcal{M} U)(\mathbf{y})=\int_{S^{2}} U(\mathbf{y}, \mathbf{n}) \mathrm{d} \sigma(\mathbf{n}) .
$$

If $U: \mathbb{R}^{3} \rtimes S^{2} \rightarrow \mathbb{R}^{+}$is a probability density on positions and orientations then $\mathcal{M U}: \mathbb{R}^{3} \rightarrow \mathbb{R}^{+}$denotes the corresponding probability density on position space only.

The marginal gives us an ordinary 3D image that is a "simplified" version of the HARDI image, containing less information on the orientational structure. This is analogue to taking the trace of a DTI image. The following theorem tells us that we get a Euclidean invariant operator on the marginal of HARDI images if the operator on the HARDI image is left-invariant. This motivates our restriction to leftinvariant operators, akin to our framework of invertible ori- 
entation scores (van Almsick 2005; Franken and Duits 2009; Franken 2008; Duits and van Almsick 2008; Duits et al. 2007; Duits and Franken 2009, 2010; Duits and Burgeth 2007).

Theorem 1 Suppose $\Phi$ is an operator on the space of HARDI images to itself. The corresponding operator $\mathcal{Y}$ on the orientation marginals given by $\mathcal{Y}(\mathcal{M}(U))=\mathcal{M}(\Phi(U))$ is Euclidean invariant if operator $\Phi$ is left-invariant:

$$
\begin{aligned}
& \left(\Phi \circ \mathfrak{L}_{g}=\mathfrak{L}_{g} \circ \Phi, \quad \text { for all } g \in \operatorname{SE}(3)\right) \\
& \quad \Rightarrow \quad \mathfrak{U}_{g} \circ \mathcal{Y}=\mathcal{Y} \circ \mathfrak{U}_{g}, \quad \text { for all } g \in \operatorname{SE}(3)
\end{aligned}
$$

Proof The result follows directly by the intertwining relation $\mathfrak{U}_{g} \circ \mathcal{M}=\mathcal{M} \circ \mathfrak{L}_{g}$ for all $g \in \operatorname{SE}(3)$. Regardless of the fact if $\Phi$ is bounded or unbounded, linear or nonlinear, we have under assumption of left-invariance of $\Phi$ that

$$
\begin{aligned}
\mathcal{Y} \circ \mathfrak{U}_{g} \circ \mathcal{M} & =\mathcal{Y} \circ \mathcal{M} \circ \mathfrak{L}_{g} \\
& =\mathcal{M} \circ \Phi \circ \mathfrak{L}_{g} \\
& =\mathcal{M} \circ \mathfrak{L}_{g} \circ \Phi=\mathfrak{U}_{g} \circ \mathcal{M} \circ \Phi \\
& =\mathfrak{U}_{g} \circ \mathcal{Y} \circ \mathcal{M} .
\end{aligned}
$$

It follows by the Dunford-Pettis Theorem (Bukhvalov and Arendt 1994, pp. 113-114) that basically every reasonable linear operator in image processing is a kernel operator. Therefore, we will classify all linear left-invariant kernel operators $\mathcal{K}$ on HARDI images and we will provide an important probabilistic interpretation of these left-invariant kernel operators.

Lemma 1 Let $\mathcal{K}$ be a bounded linear operator from $\mathbb{L}_{2}\left(\mathbb{R}^{3} \rtimes S^{2}\right)$ into $\mathbb{L}_{\infty}\left(\mathbb{R}^{3} \rtimes S^{2}\right)$ then there exists an integrable kernel $k: \mathbb{R}^{3} \rtimes S^{2} \times \mathbb{R}^{3} \rtimes S^{2} \rightarrow \mathbb{C}$ such that $\|\mathcal{K}\|^{2}=\sup _{(\mathbf{y}, \mathbf{n}) \in \mathbb{R}^{3} \rtimes S^{2}} \int_{\mathbb{R}^{3} \rtimes S^{2}}\left|k\left(\mathbf{y}, \mathbf{n} ; \mathbf{y}^{\prime}, \mathbf{n}^{\prime}\right)\right|^{2} \mathrm{~d} \mathbf{y}^{\prime} \mathrm{d} \sigma\left(\mathbf{n}^{\prime}\right)$ and we have

$$
(\mathcal{K} U)(\mathbf{y}, \mathbf{n})=\int_{\mathbb{R}^{3} \rtimes S^{2}} k\left(\mathbf{y}, \mathbf{n} ; \mathbf{y}^{\prime}, \mathbf{n}^{\prime}\right) U\left(\mathbf{y}^{\prime}, \mathbf{n}^{\prime}\right) \mathrm{d} \mathbf{y}^{\prime} \mathrm{d} \sigma\left(\mathbf{n}^{\prime}\right),
$$

for almost every $(\mathbf{y}, \mathbf{n}) \in \mathbb{R}^{3} \rtimes S^{2}$ and all $U \in \mathbb{L}_{2}\left(\mathbb{R}^{3} \rtimes S^{2}\right)$. Now $\mathcal{K}_{k}:=\mathcal{K}$ is left-invariant iff $k$ is left-invariant:

$$
\begin{aligned}
& \forall_{g \in S E(3)}: \mathfrak{L}_{g} \circ \mathcal{K}_{k}=\mathcal{K}_{k} \circ \mathfrak{L}_{g} \\
& \quad \Leftrightarrow \quad \forall_{g \in S E(3)} \forall_{\mathbf{y}, \mathbf{y}^{\prime} \in \mathbb{R}^{3} \forall_{\mathbf{n}, \mathbf{n}^{\prime} \in S^{2}}:} \\
& \quad k\left(g \cdot(\mathbf{y}, \mathbf{n}) ; g \cdot\left(\mathbf{y}^{\prime}, \mathbf{n}^{\prime}\right)\right)=k\left(\mathbf{y}, \mathbf{n} ; \mathbf{y}^{\prime}, \mathbf{n}^{\prime}\right) .
\end{aligned}
$$

Proof The first part of the lemma follows by the general Dunford-Pettis Theorem (Bukhvalov and Arendt 1994, pp. 113-114). With respect to the left-invariance we note that on the one hand we have

$$
\begin{aligned}
& \left(\mathcal{K}_{k} \mathfrak{L}_{g} U\right)(\mathbf{y}, \mathbf{n}) \\
& =\int_{S^{2}} \int_{\mathbb{R}^{3}} k\left(\mathbf{y}, \mathbf{n} ; \mathbf{y}^{\prime \prime}, \mathbf{n}^{\prime \prime}\right) \\
& \quad \times U\left(R^{-1}\left(\mathbf{y}^{\prime \prime}-\mathbf{x}\right), R^{-1} \mathbf{n}^{\prime \prime}\right) \mathrm{d} \mathbf{y}^{\prime \prime} \mathrm{d} \sigma\left(\mathbf{n}^{\prime \prime}\right) \\
& =\int_{S^{2}} \int_{\mathbb{R}^{3}} k\left(\mathbf{y}, \mathbf{n} ; R \mathbf{y}^{\prime}+\mathbf{x}, R \mathbf{n}^{\prime}\right) U\left(\mathbf{y}^{\prime}, \mathbf{n}^{\prime}\right) \mathrm{d} \mathbf{y}^{\prime} \mathrm{d} \sigma\left(\mathbf{n}^{\prime}\right) \\
& =\int_{S^{2}} \int_{\mathbb{R}^{3}} k\left(\mathbf{y}, \mathbf{n} ; g \cdot\left(\mathbf{y}^{\prime}, \mathbf{n}^{\prime}\right)\right) U\left(\mathbf{y}^{\prime}, \mathbf{n}^{\prime}\right) \mathrm{d} \mathbf{y}^{\prime} \mathrm{d} \sigma\left(\mathbf{n}^{\prime}\right)
\end{aligned}
$$

whereas on the other hand $\left(\mathfrak{L}_{g} \mathcal{K}_{k} U\right)(\mathbf{y}, \mathbf{n})=$ $\int_{S^{2}} \int_{\mathbb{R}^{3}} k\left(g^{-1}(\mathbf{y}, \mathbf{n}) ; \mathbf{y}^{\prime}, \mathbf{n}^{\prime}\right) U\left(\mathbf{y}^{\prime}, \mathbf{n}^{\prime}\right) \mathrm{d} \mathbf{y}^{\prime} \mathrm{d} \sigma\left(\mathbf{n}^{\prime}\right)$, for all $g \in$ $S E(3), U \in \mathbb{L}_{2}\left(\mathbb{R}^{3} \rtimes S^{2}\right),(\mathbf{x}, \mathbf{n}) \in \mathbb{R}^{3} \rtimes S^{2}$. Now $S E(3)$ acts transitively on $\mathbb{R}^{3} \rtimes S^{2}$ from which the result follows.

From the invariance property, (8), we deduce that

$$
\begin{aligned}
& k\left(\mathbf{y}, \mathbf{n} ; \mathbf{y}^{\prime}, \mathbf{n}^{\prime}\right) \\
& \quad=k\left(\left(R_{\mathbf{e}_{z}, \gamma^{\prime}} R_{\mathbf{e}_{y}, \beta^{\prime}}\right)^{T}\left(\mathbf{y}-\mathbf{y}^{\prime}\right),\left(R_{\mathbf{e}_{z}, \gamma^{\prime}} R_{\mathbf{e}_{y}, \beta^{\prime}}\right)^{T} \mathbf{n} ; \mathbf{0}, \mathbf{e}_{z}\right), \\
& k\left(R_{\mathbf{e}_{z}, \alpha} \mathbf{y}, R_{\mathbf{e}_{z}, \alpha} \mathbf{n} ; \mathbf{0}, \mathbf{e}_{z}\right)=k\left(\mathbf{y}, \mathbf{n} ; \mathbf{0}, \mathbf{e}_{z}\right)
\end{aligned}
$$

and consequently we obtain the following result:

Corollary 1 By the well-known Euler-angle parametrization of $S O(3)$, we have $S O(3) / S O(2) \equiv S^{2}$ via isomorphism $\left[R_{\mathbf{e}_{z}, \gamma} R_{\mathbf{e}_{y}, \beta}\right]=\left\{R_{\mathbf{e}_{z}, \gamma} R_{\mathbf{e}_{y}, \beta} R_{\mathbf{e}_{z}, \alpha} \mid \alpha \in[0,2 \pi)\right\} \leftrightarrow$ $\mathbf{n}(\beta, \gamma)=(\sin \beta \cos \gamma, \sin \beta \sin \gamma, \cos \beta)^{T}=R_{\mathbf{e}_{z}, \gamma} R_{\mathbf{e}_{y}, \beta} \mathbf{e}_{z}$. To each positive left-invariant kernel $k: \mathbb{R}^{3} \rtimes S^{2} \times \mathbb{R}^{3} \rtimes$ $S^{2} \rightarrow \mathbb{R}^{+}$with $\int_{S^{2}} \int_{\mathbb{R}^{3}} k\left(\mathbf{0}, \mathbf{e}_{z} ; \mathbf{y}, \mathbf{n}\right) \mathrm{d} \mathbf{y d} \sigma(\mathbf{n})=1$ we associate a unique probability density $p: \mathbb{R}^{3} \rtimes S^{2} \rightarrow \mathbb{R}^{+}$with the invariance property

$p(\mathbf{y}, \mathbf{n})=p\left(R_{\mathbf{e}_{z}, \alpha} \mathbf{y}, R_{\mathbf{e}_{z}, \alpha} \mathbf{n}\right), \quad$ for all $\alpha \in[0,2 \pi)$,

such that

$$
\begin{aligned}
& k\left(\mathbf{y}, \mathbf{n}(\beta, \gamma) ; \mathbf{y}^{\prime}, \mathbf{n}\left(\beta^{\prime}, \gamma^{\prime}\right)\right) \\
& \quad=p\left(\left(R_{\mathbf{e}_{z}, \gamma^{\prime}} R_{\mathbf{e}_{y}, \beta^{\prime}}\right)^{T}\left(\mathbf{y}-\mathbf{y}^{\prime}\right),\left(R_{\mathbf{e}_{z}, \gamma^{\prime}} R_{\mathbf{e}_{y}, \beta^{\prime}}\right)^{T} \mathbf{n}(\beta, \gamma)\right)
\end{aligned}
$$

with $p(\mathbf{y}, \mathbf{n})=k\left(\mathbf{y}, \mathbf{n} ; \mathbf{0}, \mathbf{e}_{z}\right)$. We can briefly rewrite $($ Franken 2008, (7.59)) and (7), coordinate-independently, as

$$
\begin{aligned}
& \mathcal{K}_{k} U(\mathbf{y}, \mathbf{n})=\left(p *_{\mathbb{R}^{3} \rtimes S^{2}} U\right)(\mathbf{y}, \mathbf{n}) \\
& \quad=\int_{\mathbb{R}^{3}} \int_{S^{2}} p\left(R_{\mathbf{n}^{\prime}}^{T}\left(\mathbf{y}-\mathbf{y}^{\prime}\right), R_{\mathbf{n}^{\prime}}^{T} \mathbf{n}\right) U\left(\mathbf{y}^{\prime}, \mathbf{n}^{\prime}\right) \mathrm{d} \sigma\left(\mathbf{n}^{\prime}\right) \mathrm{d} \mathbf{y}^{\prime},
\end{aligned}
$$

where $\sigma$ denotes the surface measure on the sphere and where $R_{\mathbf{n}^{\prime}}$ is any rotation such that $\mathbf{n}^{\prime}=R_{\mathbf{n}^{\prime}} \mathbf{e}_{z}$. 
By the invariance property (9), the convolution (10) on $\mathbb{R}^{3} \rtimes S^{2}$ may be written as a (full) $S E$ (3)-convolution. An $S E$ (3) convolution (Chirikjian and Kyatkin 2001) of two functions $\tilde{p}: S E(3) \rightarrow \mathbb{R}, \tilde{U}: S E(3) \rightarrow \mathbb{R}$ is given by:

$$
\left(\tilde{p} *_{S E(3)} \tilde{U}\right)(g)=\int_{S E(3)} \tilde{p}\left(h^{-1} g\right) \tilde{U}(h) \mathrm{d} \mu_{S E(3)}(h),
$$

where Haar-measure $\mathrm{d} \mu_{S E(3)}(\mathbf{x}, R)=\mathrm{d} \mathbf{x} \mathrm{d} \mu_{S O(3)}(R)$ with $\mathrm{d} \mu_{S O(3)}\left(R_{\mathbf{e}_{z}, \gamma} R_{\mathbf{e}_{y}, \beta} R_{\mathbf{e}_{z}, \alpha}\right)=\sin \beta \mathrm{d} \alpha \mathrm{d} \beta \mathrm{d} \gamma$. If we now set $\tilde{p}(\mathbf{x}, R):=p\left(\mathbf{x}, R \mathbf{e}_{z}\right)$ and $\tilde{U}(\mathbf{x}, R):=U\left(\mathbf{x}, R \mathbf{e}_{z}\right)$, it follows by (9) that the following identity holds:

$$
\left(\tilde{p} *_{S E(3)} \tilde{U}\right)(\mathbf{x}, R)=2 \pi\left(p *_{\mathbb{R}^{3} \rtimes S^{2}} U\right)\left(\mathbf{x}, R \mathbf{e}_{z}\right) .
$$

Later on in this article (in Sects. 4.2 and 4.3) we will relate scale spaces on HARDI data and first order Tikhonov regularization on HARDI data to Markov processes. But in order to provide a road map of how the $\mathbb{R}^{3} \rtimes S^{2}$-convolutions will appear in the more technical remainder of this article we provide some preliminary explanations on probabilistic interpretation of $\mathbb{R}^{3} \rtimes S^{2}$-convolutions.

In particular we will restrict ourselves to conditional probabilities where $p(\mathbf{y}, \mathbf{n})=p_{t}(\mathbf{y}, \mathbf{n})$ represents the probability density of finding an oriented random walker at position $\mathbf{y}$ with orientation $\mathbf{n}$ at time $t>0$, given that it started at $\left(\mathbf{0}, \mathbf{e}_{z}\right)$ at time $t=0$. In such a case the probabilistic interpretation of the kernel operator is as follows. The function $(\mathbf{y}, \mathbf{n}) \mapsto\left(\mathcal{K}_{k_{t}} U\right)(\mathbf{y}, \mathbf{n})=\left(p_{t} *_{\mathbb{R}^{3} \rtimes S^{2}} U\right)(\mathbf{y}, \mathbf{n})$ represents the probability density of finding some oriented particle, starting from the initial distribution $U: \mathbb{R}^{3} \rtimes S^{2} \rightarrow \mathbb{R}^{+}$at time $t=0$, at location $\mathbf{y} \in \mathbb{R}^{3}$ with orientation $\mathbf{n} \in S^{2}$ at time $t>0$. Furthermore, in a Markov process traveling time is memoryless, so in such process traveling time is negatively exponentially distributed $P(T=t)=\lambda e^{-\lambda t}$ with expectation $E(T)=\lambda^{-1}$. Consequently, the probability density $p^{\lambda}$ of finding an oriented random walker starting from $\left(\mathbf{0}, \mathbf{e}_{z}\right)$ at time $t=0$, regardless its traveling time equals

$$
\begin{aligned}
p^{\lambda}(\mathbf{y}, \mathbf{n}) & =\int_{0}^{\infty} p_{t}(\mathbf{y}, \mathbf{n}) P(T=t) \mathrm{d} t \\
& =\lambda \int_{0}^{\infty} p_{t}(\mathbf{y}, \mathbf{n}) e^{-\lambda t} \mathrm{~d} t .
\end{aligned}
$$

Summarizing, we can always apply Laplace-transform with respect to time to map transition densities $p_{t}(g)$ given a traveling time $t>0$ to unconditional probability densities $p^{\lambda}(g)$. The same holds for the probability density $P^{\lambda}(\mathbf{y}, \mathbf{n})$ of finding an oriented random walker at location $\mathbf{y} \in \mathbb{R}^{3}$ with orientation $\mathbf{n} \in S^{2}$ starting from initial distribution $U$ (i.e. the HARDI data) regardless the traveling time, since

$$
\begin{aligned}
P_{U}^{\lambda}(\mathbf{y}, \mathbf{n}) & =\lambda \int_{0}^{\infty} e^{-\lambda t}\left(p_{t} *_{\mathbb{R}^{3} \rtimes S^{2}} U\right)(\mathbf{y}, \mathbf{n}) \mathrm{d} t \\
& =\left(p^{\lambda} *_{\mathbb{R}^{3} \rtimes S^{2}} U\right)(\mathbf{y}, \mathbf{n}) .
\end{aligned}
$$

3.1 Relation of the Method Proposed by Barmpoutis et al. to $\mathbb{R}^{3} \rtimes S^{2}$-Convolution

In Barmpoutis et al. (2008) the authors propose ${ }^{2}$ the following practical decomposition for the kernel $k$ :

$$
\begin{aligned}
k^{t, \kappa}\left(\mathbf{y}, \mathbf{n} ; \mathbf{y}^{\prime}, \mathbf{n}^{\prime}\right)= & \frac{1}{4 \pi} k_{\text {dist }}^{t}\left(\left\|\mathbf{y}-\mathbf{y}^{\prime}\right\|\right) \cdot k_{\text {orient }}^{\kappa}\left(\mathbf{n} \cdot \mathbf{n}^{\prime}\right) \\
& \cdot k_{\text {fiber }}^{\kappa}\left(\frac{1}{\left\|\mathbf{y}-\mathbf{y}^{\prime}\right\|} \mathbf{n} \cdot\left(-\left(\mathbf{y}-\mathbf{y}^{\prime}\right)\right)\right),
\end{aligned}
$$

with $k_{\text {dist }}^{t}\left(\left\|\mathbf{y}-\mathbf{y}^{\prime}\right\|\right)=\frac{1}{(4 \pi t)^{\frac{3}{2}}} e^{-\frac{\left\|\mathbf{y}-\mathbf{y}^{\prime}\right\|^{2}}{4 t}}$ and $k_{\text {orient }}^{\kappa}(\cos \phi)=$ $k_{\text {fiber }}^{\kappa}(\cos \phi)=\frac{e^{\kappa \cos (\phi)}}{2 \pi J_{0}(i \kappa)}$ with $\phi \in(-\pi, \pi]$ the angle, respectively, between the vectors $\mathbf{n}$ and $\mathbf{n}^{\prime}$ and the angle between the vectors $\mathbf{n}$ and $-\left(\mathbf{y}-\mathbf{y}^{\prime}\right)$. So $k_{\text {orient }}^{\kappa}(\cos \phi)$ denotes the von Mises distribution on the circle, which is indeed positive and $\int_{-\pi}^{\pi} \frac{e^{\kappa \cos (\phi)}}{2 \pi J_{0}(i \kappa)} \mathrm{d} \phi=1$. The decomposition (14) automatically implies that the corresponding kernel operator $\mathcal{K}_{k}$ is left-invariant, regardless the choice of $k_{\text {dist }}, k_{\text {orient }}^{\kappa}, k_{\text {fiber }}^{\kappa}$ since

$$
\begin{aligned}
& k_{\text {dist }}^{s}(\left.\left\|R^{-1}(\mathbf{y}-\mathbf{x})-R^{-1}\left(\mathbf{y}^{\prime}-\mathbf{x}\right)\right\|\right) \cdot k_{\text {orient }}^{\kappa}\left(R^{-1} \mathbf{n} \cdot R^{-1} \mathbf{n}^{\prime}\right) \\
& \cdot k_{\text {fiber }}^{\kappa}\left(-\frac{1}{\left\|R^{-1}(\mathbf{y}-\mathbf{x})-R^{-1}\left(\mathbf{y}^{\prime}-\mathbf{x}\right)\right\|}\left(R^{-1} \mathbf{n}^{\prime}\right)\right. \\
&\left.\cdot R^{-1}\left(\mathbf{y}-\mathbf{x}-\left(\mathbf{y}^{\prime}-\mathbf{x}\right)\right)\right) \\
&= k_{\text {dist }}^{t}\left(\left\|\mathbf{y}-\mathbf{y}^{\prime}\right\|\right) \cdot k_{\text {orient }}^{\kappa}\left(\mathbf{n} \cdot \mathbf{n}^{\prime}\right) \\
& \cdot k_{\text {fiber }}^{\kappa}\left(-\frac{1}{\left\|\mathbf{y}-\mathbf{y}^{\prime}\right\|} \mathbf{n} \cdot\left(\mathbf{y}-\mathbf{y}^{\prime}\right)\right) \Leftrightarrow \\
& k^{t, \kappa}\left(g^{-1}(\mathbf{y}, \mathbf{n}) ; g^{-1}\left(\mathbf{y}^{\prime}, \mathbf{n}^{\prime}\right)\right)=k^{t, \kappa}\left(\mathbf{y}, \mathbf{n} ; \mathbf{y}^{\prime}, \mathbf{n}^{\prime}\right),
\end{aligned}
$$$$
\text { for all } g=(\mathbf{x}, R) \in \operatorname{SE}(3) \text {. }
$$

The corresponding probability kernel (which does satisfy (9)) reads

$$
\begin{aligned}
& p_{(t, \kappa)}(\mathbf{y}, \mathbf{n})=\frac{1}{4 \pi} k_{\text {dist }}^{t}(\|\mathbf{y}\|) k_{\text {orient }}^{\kappa}\left(\mathbf{e}_{z} \cdot \mathbf{n}\right) k_{\text {fiber }}^{\kappa}\left(-\|\mathbf{y}\|^{-1} \mathbf{n} \cdot \mathbf{y}\right), \\
& \quad \mathbf{y} \neq 0 .
\end{aligned}
$$

For a simple probabilistic interpretation we apply a spatial reflection ${ }^{3}$ and define $p_{(t, \kappa)}^{+}(\mathbf{y}, \mathbf{n})=p_{(t, \kappa)}(-\mathbf{y}, \mathbf{n})$. Now $p_{(t, \kappa)}^{+}$should be interpreted as a probability density of finding an oriented particle at position $\mathbf{y} \in \mathbb{R}^{3}$ with orientation $\mathbf{n} \in S^{2}$ given that it started at position $\mathbf{0}$ with orientation $\mathbf{e}_{z}$. The practical rationale behind the decomposition (14), is that two neighboring local orientations $(\mathbf{y}, \mathbf{n}) \in$

\footnotetext{
${ }^{2}$ We used slightly different conventions as in the original paper to ensure $\mathbb{L}_{1}$-normalizations in (14).

${ }^{3}$ Later on in Sect. 8.2.1 we will return to the important practical consequences of this spatial reflection in full detail.
} 
$\mathbb{R}^{3} \rtimes S^{2}$ and $\left(\mathbf{y}^{\prime}, \mathbf{n}^{\prime}\right) \in \mathbb{R}^{3} \rtimes S^{2}$ are supposed to strengthen each other if the distance between $\mathbf{y}$ and $\mathbf{y}^{\prime}$ is close (represented by the first kernel $k_{\text {dist }}$ ), if moreover the orientations $\mathbf{n}, \mathbf{n}^{\prime}$ are similar (represented by $k_{\text {orient }}^{\kappa}$ ), and finally if local orientation $(\mathbf{y}, \mathbf{n})$ is nicely aligned according to some a priori fibre model with the local orientation $\left(\mathbf{y}^{\prime}, \mathbf{n}^{\prime}\right)$, i.e. if the orientation of $\left\|\mathbf{y}-\mathbf{y}^{\prime}\right\|^{-1}\left(\mathbf{y}-\mathbf{y}^{\prime}\right)$ is close to the orientation $\mathbf{n}$ (represented by $k_{\text {fiber }}^{\kappa}$ ). The decomposition allows a reduction of computation via:

$$
\begin{aligned}
\left(\mathcal{K}_{k^{t, \kappa}} U\right)(\mathbf{y}, \mathbf{n}) \\
=\left(p_{t, \kappa} *_{\mathbb{R}^{3} \rtimes S^{2}} U\right)(\mathbf{y}, \mathbf{n}) \\
=\frac{1}{4 \pi} \int_{\mathbb{R}^{3}} k_{\mathrm{dist}}^{t}\left(\left\|\mathbf{y}-\mathbf{y}^{\prime}\right\|\right) k_{\mathrm{fiber}}^{\kappa}\left(\left\|\mathbf{y}-\mathbf{y}^{\prime}\right\|^{-1} \mathbf{n} \cdot\left(\mathbf{y}^{\prime}-\mathbf{y}\right)\right) \\
\quad \cdot\left(\int_{S^{2}} U\left(\mathbf{y}^{\prime}, \mathbf{n}^{\prime}\right) k_{\text {orient }}^{\kappa}\left(\mathbf{n} \cdot \mathbf{n}^{\prime}\right) \mathrm{d} \sigma\left(\mathbf{n}^{\prime}\right)\right) \mathrm{d} \mathbf{y}^{\prime} .
\end{aligned}
$$

Despite the fact that the practical kernel in (14) gives rise to a reasonable connectivity measure between two local orientations $(\mathbf{y}, \mathbf{n})$ and $\left(\mathbf{y}^{\prime}, \mathbf{n}^{\prime}\right) \in \mathbb{R}^{3} \rtimes S^{2}$ and that the associated kernel operator has the right covariance properties, the associated kernel operator is not related to left-invariant diffusion and/or Tikhonov regularization on $\mathbb{R}^{3} \rtimes S^{2}$, as was aimed for in the paper (Barmpoutis et al. 2008). In this inspiring pioneering paper the authors consider a position dependent energy and deal with the Euler-Lagrange equations in an unusual way (in particular Barmpoutis et al. 2008, (7)). The kernel given by (15) involves two separate time parameters $t, \kappa$ and the probability kernels given by (14) are not related to Brownian motions and/or Markov-processes on $\mathbb{R}^{3} \rtimes S^{2}$, since they do not satisfy the semigroup property. A disadvantage as we will explain next, however, is that the kernel is not entirely suited for iteration unless combined with nonlinear operators such as nonlinear grey-value transformations. The function $\mathbf{y} \mapsto\|\mathbf{y}\|^{-1} \mathbf{y} \cdot \mathbf{n}$ within (15) is discontinuous at the origin. If the origin is approached by a straight-line along $\mathbf{n}$ the limit-value is 1 and this seems to be a reasonable choice for evaluating the kernel at $\mathbf{y}=\mathbf{0}$. The finite maximum of the kernel is now obtained at $\mathbf{y}=\mathbf{0}$ (and $\mathbf{n}=\mathbf{e}_{z}$ ). Since the kernel is single-sided and does not have a singularity at the origin convolution with itself will allow the maximum of the effective kernel to run away ${ }^{4}$ from its center. See Fig. 5, where we numerically $\mathbb{R}^{3} \rtimes S^{2}$-convolved the kernel given in (14) with itself by a convolution algorithm that we will explain later in Sect. 8.2. However, if the kernel would have satisfied the semigroup-property such artifacts would not have occurred. For example the single-sided exact Green's function of Mumford's direction

${ }^{4}$ Set $\mathbf{a}:=\frac{1}{1+\alpha}[\ldots, 0,1, \alpha, 0, \ldots]$, then for every $n \in \mathbb{N}$ the sequence $\mathbf{a}_{n}:=\mathbf{a} *^{(n-1)} \mathbf{a} \in \ell_{1}(\mathbb{Z})$ has $n+1$ non-zero coefficients: $a_{n}^{k}=(1+\alpha)^{-n} \alpha^{k}\left(\begin{array}{l}n \\ k\end{array}\right), k=0, \ldots, n$. So the position of the maximum of $\mathbf{a}_{n}$ increases with $n$ (if $\alpha=1$ it takes place at $k=\left\lfloor\frac{n}{2}\right\rfloor$ ).

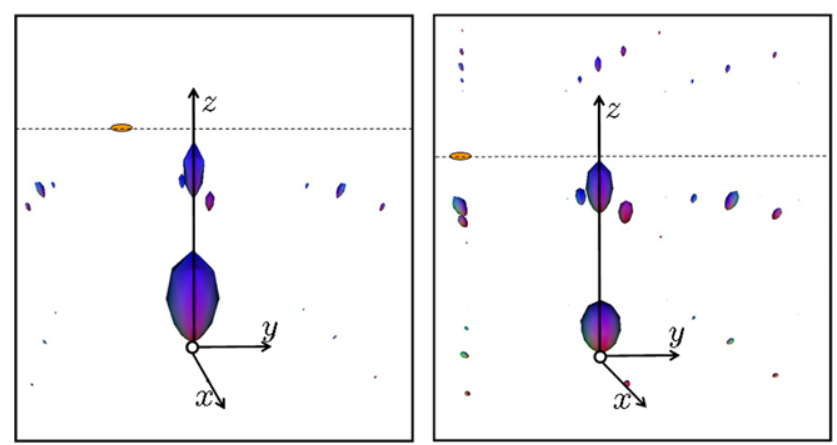

Fig. 5 Left: Glyph visualization (recall Definition 1) of the kernel $p_{(t, \kappa)}^{+}: \mathbb{R}^{3} \rtimes S^{2} \rightarrow \mathbb{R}^{+}(15)$ as proposed in Barmpoutis et al. (2008), plotted in perspective with respect to indicated horizon (dashed line) and vanishing point. Right: Glyph visualization of $p_{(t, \kappa)}^{+} *_{\mathbb{R}^{3} \rtimes S^{2}} p_{(t, \kappa)}^{+}$, i.e. the kernel numerically convolved with itself (kernels are sampled on a $3 \times 3 \times 3$-grid with 162-orientations). Parameter settings are $\left(t=\frac{1}{2}, \kappa=4\right)$. The maximum moves away from the origin by iteration: in the right image the second glyph on the $z$-axis has a larger radius than the glyph at $\mathbf{0}$. The effective shape of the convolution kernel is destroyed by iteration, as the kernel in (15) does not satisfy the semigroup property. This motivates our quest (in Sect. 6) for appropriate diffusion kernels (related to Brownian motion on $\mathbb{R}^{3} \rtimes S^{2}$ ) on $\mathbb{R}^{3} \rtimes S^{2}$ that do satisfy the semigroup property $p_{t} *_{\mathbb{R}^{3} \rtimes S^{2}} p_{s}=p_{s+t}$

process (Duits and van Almsick 2008) (and its approximations Thornber and Williams 2000; Duits and Franken 2009; Duits and van Almsick 2008) on $S E(2)=\mathbb{R}^{2} \rtimes S^{1}$ has a natural singularity at the origin.

Before we consider scale spaces on HARDI data whose solutions are given by $\mathbb{R}^{3} \rtimes S^{2}$-convolution, (10), with the corresponding Green's functions (which do satisfy the semigroup-property) we provide, for the sake of clarity, a quick review on scale spaces of periodic signals from a group theoretical PDE-point of view.

\subsection{Introductory Example: Scale Space and Tikhonov \\ Regularization on the Circle}

The Gaussian scale space equation and corresponding resolvent equation (i.e. the solution of Tikhonov regularization) on a circle $\mathbb{T}=\left\{e^{i \theta} \mid \theta \in[0,2 \pi)\right\} \equiv S^{1}$ with group product $e^{i \theta} e^{i \theta^{\prime}}=e^{i\left(\theta+\theta^{\prime}\right)}$, are given by

$$
\begin{aligned}
& \left\{\begin{array}{l}
\partial_{t} u(\theta, t)=D_{11} \partial_{\theta}^{2} u(\theta, t), \\
u(0, t)=u(2 \pi, t) \quad \text { and } \quad u(\theta, 0)=f(\theta)
\end{array}\right. \text { and } \\
& p_{\gamma}(\theta)=\gamma\left(D_{11} \partial_{\theta}^{2}-\gamma I\right)^{-1} f(\theta),
\end{aligned}
$$

with $\theta \in[0,2 \pi)$ and $D_{11}>0$ fixed, where we note that the function $\theta \mapsto p_{\gamma}(\theta)=\gamma \int_{0}^{\infty} u(\theta, t) e^{-\gamma t} \mathrm{~d} t$ is the minimizer of the Tikhonov-energy

$\mathcal{E}\left(p_{\gamma}\right):=\int_{0}^{2 \pi} \gamma\left|p_{\gamma}(\theta)-f(\theta)\right|^{2}+D_{11}\left|p_{\gamma}^{\prime}(\theta)\right|^{2} \mathrm{~d} \theta$

under the periodicity condition $p_{\gamma}(0)=p_{\gamma}(2 \pi)$. By leftinvariance the solutions are given by $\mathbb{T}$-convolution 
with their Green's function, say $G_{t}^{D_{11}}: \mathbb{T} \rightarrow \mathbb{R}^{+}$and $R_{\gamma}^{D_{11}}: \mathbb{T} \rightarrow \mathbb{R}^{+}$. Recall that the relation between Tikhonov regularization and scale space theory is given by Laplacetransform with respect to time:

$$
\begin{aligned}
& u(\cdot, t)=e^{t \Delta_{\mathbb{T}}} f:=G_{t} *_{\mathbb{T}} f \quad \text { and } \quad p_{\gamma}=R_{\gamma}^{D_{11}} *_{\mathbb{T}} f, \\
& \text { with } R_{\gamma}^{D_{11}}=\gamma \int_{0}^{\infty} G_{t}^{D_{11}} e^{-\gamma t} \mathrm{~d} t,
\end{aligned}
$$

where the $\mathbb{T}$-convolution is given by $(f * \mathbb{T} g)\left(e^{i \theta}\right)=$ $\int_{-\pi}^{\pi} f\left(e^{i\left(\theta-\theta^{\prime}\right)}\right) g\left(e^{i \theta^{\prime}}\right) \mathrm{d} \theta^{\prime}$. For explicit formulas of the Green's function $G_{t}^{D_{11}}$ (basically a sum of $2 \pi$-shifted Gaussians) and the Green's function $R_{\gamma}^{D_{11}}$ see Duits and Franken (2009, Chap. 3.2). Now by $e^{s \Delta_{\mathbb{T}}} e^{t \Delta_{\mathbb{T}}}=e^{(s+t) \Delta_{\mathbb{T}}}$ the heatkernel on $\mathbb{T}$ satisfies the (for iterations) important semigroup property:

$G_{s}^{D_{11} *_{\mathbb{T}}} G_{t}^{D_{11}}=G_{s+t}^{D_{11}}, \quad$ for all $s, t>0$.

The generator of a Gaussian scale space on the torus is given by $D_{11} \partial_{\theta}^{2}$. Just like the solution operator $\left(D_{11} \partial_{\theta}^{2}-\lambda I\right)^{-1}$ of Tikhonov regularization, it is left-invariant on the group $\mathbb{T}$ and thereby the solutions (18) of a Gaussian Scale Space and Tikhonov regularization are given by $\mathbb{T}$-convolution. In order to generalize scale space representations of functions on a torus to scale space representations of HARDI data defined on $\mathbb{R}^{3} \rtimes S^{2}$ (embedded in $S E(3)=\mathbb{R}^{3} \rtimes S O(3)$ ), we simply have to replace the left-invariant vector field $\partial_{\theta}$ on $\mathbb{T}$ by the left-invariant vector fields on $S E(3)$ (or rather $\mathbb{R}^{3} \rtimes S^{2}$ ) in the quadratic form which generates the scale space on the group, (Duits and Burgeth 2007). This motivates the technical derivations of the left-invariant vector fields on $S E(3)$ in the next subsection.

\subsection{Left-invariant Vector Fields on $S E(3)$ and their Dual Elements}

We will use the following basis for the tangent space $T_{e}(S E(3))$ at the unity element $e=(\mathbf{0}, I) \in S E(3)$ :

$$
\begin{array}{lll}
A_{1}=\partial_{x}, & A_{2}=\partial_{y}, & A_{3}=\partial_{z}, \\
A_{4}=\partial_{\tilde{\gamma}}, & A_{5}=\partial_{\tilde{\beta}}, & A_{6}=\partial_{\tilde{\alpha}},
\end{array}
$$

where we stress that at the unity element $(\mathbf{0}, R=I)$, we have $\beta=0$ and here the tangent vectors $\partial_{\beta}$ and $\partial_{\gamma}$ are not defined, which requires a description of the tangent vectors on the $S O(3)$-part by means of the second chart.

The tangent space at the unity element is a $6 \mathrm{D}$ Lie algebra equipped with Lie bracket

$$
[A, B]=\lim _{t \downarrow 0} t^{-2}\left(a(t) b(t)(a(t))^{-1}(b(t))^{-1}-e\right),
$$

where $t \mapsto a(t)$ resp. $t \mapsto b(t)$ are any smooth curves in $S E(3)$ with $a(0)=b(0)=e$ and $a^{\prime}(0)=A$ and $b^{\prime}(0)=B$, for explanation on the formula (20) which holds for general matrix Lie groups, see Duits et al. (2009, App. G). The Liebrackets of the basis given in (19) are given by

$\left[A_{i}, A_{j}\right]=\sum_{k=1}^{6} c_{i j}^{k} A_{k}$,

where the non-zero structure constants for all three isomorphic Lie-algebras are given by

$-c_{j i}^{k}=c_{i j}^{k}=\left\{\begin{array}{c}\operatorname{sgn} \operatorname{perm}\{i-3, j-3, k-3\} \\ \text { if } i, j, k \geq 4, i \neq j \neq k, \\ \operatorname{sgn} \operatorname{perm}\{i, j-3, k\} \\ \text { if } i, k \leq 3, j \geq 4, i \neq j \neq k .\end{array}\right.$

More explicitly, we have the following table of Lie-brackets:

$$
\begin{aligned}
& \left(\left[A_{i}, A_{j}\right]\right)_{j=1, \ldots, 6}^{i=1, \ldots 6} \\
& \quad=\left(\begin{array}{cccccc}
0 & 0 & 0 & 0 & A_{3} & -A_{2} \\
0 & 0 & 0 & -A_{3} & 0 & A_{1} \\
0 & 0 & 0 & A_{2} & -A_{1} & 0 \\
0 & A_{3} & -A_{2} & 0 & A_{6} & -A_{5} \\
-A_{3} & 0 & A_{1} & -A_{6} & 0 & A_{4} \\
A_{2} & -A_{1} & 0 & A_{5} & -A_{4} & 0
\end{array}\right),
\end{aligned}
$$

so for example $c_{15}^{3}=1, c_{14}^{3}=c_{15}^{2}=0, c_{16}^{2}=-c_{61}^{2}=-1$. The corresponding left-invariant vector fields $\left\{\mathcal{A}_{i}\right\}_{i=1}^{6}$ are obtained by the push-forward of the left-multiplication $L_{g} h=g h$ by $\left.\mathcal{A}_{i}\right|_{g} \phi=\left(L_{g}\right)_{*} A_{i} \phi=A_{i}\left(\phi \circ L_{g}\right)$ (for all smooth $\phi: \Omega_{g} \rightarrow \mathbb{R}$ which are locally defined on some neighborhood $\Omega_{g}$ of $g$ ) and they can be obtained by the derivative of the right-regular representation:

$$
\left.\mathcal{A}_{i}\right|_{g} \phi=\left(\mathrm{d} \mathcal{R}\left(A_{i}\right) \phi\right)(g)=\lim _{t \downarrow 0} \frac{\phi\left(g e^{t A_{i}}\right)-\phi(g)}{t},
$$$$
\text { with } \mathcal{R}_{g} \phi(h)=\phi(h g) \text {. }
$$

Expressed in the first coordinate chart, (1), this renders for the left-invariant derivatives at position $g=(x, y, z$, $R_{\mathbf{e}_{z}, \gamma} R_{\mathbf{e}_{y}, \beta} R_{\mathbf{e}_{z}, \alpha}$ ) $\in S E$ (3) (see also Chirikjian and Kyatkin 2001, Sect. 9.10)

$$
\begin{aligned}
\mathcal{A}_{1}= & (\cos \alpha \cos \beta \cos \gamma-\sin \alpha \sin \gamma) \partial_{x} \\
& +(\sin \alpha \cos \gamma+\cos \alpha \cos \beta \sin \gamma) \partial_{y}-\cos \alpha \sin \beta \partial_{z}, \\
\mathcal{A}_{2}= & (-\sin \alpha \cos \beta \cos \gamma-\cos \alpha \sin \gamma) \partial_{x} \\
& +(\cos \alpha \cos \gamma-\sin \alpha \cos \beta \sin \gamma) \partial_{y} \\
& +\sin \alpha \sin \beta \partial_{z}, \\
\mathcal{A}_{3}= & \sin \beta \cos \gamma \partial_{x}+\sin \beta \sin \gamma \partial_{y}+\cos \beta \partial_{z}, \\
\mathcal{A}_{4}= & \cos \alpha \cot \beta \partial_{\alpha}+\sin \alpha \partial_{\beta}-\frac{\cos \alpha}{\sin \beta} \partial_{\gamma}, \\
\mathcal{A}_{5}= & -\sin \alpha \cot \beta \partial_{\alpha}+\cos \alpha \partial_{\beta}+\frac{\sin \alpha}{\sin \beta} \partial_{\gamma}, \\
\mathcal{A}_{6}= & \partial_{\alpha} .
\end{aligned}
$$


for $\beta \neq 0$ and $\beta \neq \pi$. The explicit formulae of the leftinvariant vector fields (which are well-defined in north- and south-pole) in the second chart, (4), are:

$$
\begin{aligned}
\mathcal{A}_{1}= & \cos \tilde{\alpha} \cos \tilde{\beta} \partial_{x}+(\cos \tilde{\gamma} \sin \tilde{\alpha}+\cos \tilde{\alpha} \sin \tilde{\beta} \sin \tilde{\gamma}) \partial_{y} \\
& +(\sin \tilde{\alpha} \sin \tilde{\gamma}-\cos \tilde{\alpha} \cos \tilde{\gamma} \sin \tilde{\beta}) \partial_{z}, \\
\mathcal{A}_{2}= & -\sin \tilde{\alpha} \cos \tilde{\beta} \partial_{x}+(\cos \tilde{\alpha} \cos \tilde{\gamma}-\sin \tilde{\alpha} \sin \tilde{\beta} \sin \tilde{\gamma}) \partial_{y} \\
& +(\sin \tilde{\alpha} \sin \tilde{\beta} \cos \tilde{\gamma}+\cos \tilde{\alpha} \sin \tilde{\gamma}) \partial_{z}, \\
\mathcal{A}_{3}= & \sin \tilde{\beta} \partial_{x}-\cos \tilde{\beta} \sin \tilde{\gamma} \partial_{y}+\cos \tilde{\beta} \cos \tilde{\gamma} \partial_{z}, \\
\mathcal{A}_{4}= & -\cos \tilde{\alpha} \tan \tilde{\beta} \partial_{\tilde{\alpha}}+\sin \tilde{\alpha} \partial_{\tilde{\beta}}+\frac{\cos \tilde{\alpha}}{\cos \tilde{\beta}} \partial_{\tilde{\gamma}}, \\
\mathcal{A}_{5}= & \sin \tilde{\alpha} \tan \tilde{\beta} \partial_{\tilde{\alpha}}+\cos \tilde{\alpha} \partial_{\tilde{\beta}}-\frac{\sin \tilde{\alpha}}{\cos \tilde{\beta}} \partial_{\tilde{\gamma}}, \\
\mathcal{A}_{6}= & \partial_{\tilde{\alpha}},
\end{aligned}
$$

for $\tilde{\beta} \neq \frac{\pi}{2}$ and $\tilde{\beta} \neq-\frac{\pi}{2}$. Note that $\mathrm{d} \mathcal{R}$ is a Lie-algebra isomorphism, i.e.

$$
\begin{aligned}
& {\left[A_{i}, A_{j}\right]=\sum_{k=1}^{6} c_{i j}^{k} A_{k}} \\
& \Leftrightarrow \quad\left[\mathrm{d} \mathcal{R}\left(A_{i}\right), \mathrm{d} \mathcal{R}\left(A_{j}\right)\right]=\sum_{k=1}^{6} c_{i j}^{k} \mathrm{~d} \mathcal{R}\left(A_{k}\right) \\
& \Leftrightarrow \quad\left[\mathcal{A}_{i}, \mathcal{A}_{j}\right]=\mathcal{A}_{i} \mathcal{A}_{j}-\mathcal{A}_{j} \mathcal{A}_{i}=\sum_{k=1}^{6} c_{i j}^{k} \mathcal{A}_{k} .
\end{aligned}
$$

These vector fields form a local moving coordinate frame of reference on $S E(3)$, the corresponding dual frame $\left\{\mathrm{d} \mathcal{A}^{1}, \ldots, \mathrm{d} \mathcal{A}^{6}\right\} \in(T(S E(3)))^{*}$ is defined by

$$
\left\langle\mathrm{d} \mathcal{A}^{i}, \mathcal{A}_{j}\right\rangle:=\mathrm{d} \mathcal{A}^{i}\left(\mathcal{A}_{j}\right)=\delta_{j}^{i}, \quad i, j=1, \ldots, 6,
$$

where $\delta_{j}^{i}=1$ if $i=j$ and zero else. A brief computation yields the following dual frame (in both coordinate charts):

$$
\begin{aligned}
\left(\begin{array}{l}
\mathrm{d} \mathcal{A}^{1} \\
\mathrm{~d} \mathcal{A}^{2} \\
\mathrm{~d} \mathcal{A}^{3} \\
\mathrm{~d} \mathcal{A}^{4} \\
\mathrm{~d} \mathcal{A}^{5} \\
\mathrm{~d} \mathcal{A}^{6}
\end{array}\right) & =\left(\begin{array}{c|c}
\left(R_{\mathbf{e}_{z}, \gamma} R_{\mathbf{e}_{y}, \beta} R_{\mathbf{e}_{z}, \alpha}\right)^{T} & \mathbf{0} \\
\hline \mathbf{0} & M_{\beta, \alpha}
\end{array}\right)\left(\begin{array}{c}
\mathrm{d} x \\
\mathrm{~d} y \\
\mathrm{~d} z \\
\mathrm{~d} \alpha \\
\mathrm{d} \beta \\
\mathrm{d} \gamma
\end{array}\right) \\
& =\left(\begin{array}{c|c}
\left(R_{\mathbf{e}_{x}, \tilde{\gamma}} R_{\mathbf{e}_{y}, \tilde{\beta}} R_{\mathbf{e}_{z}, \tilde{\alpha}}\right)^{T} & \mathbf{0} \\
\hline \mathbf{0} & \tilde{M}_{\tilde{\beta}, \tilde{\alpha}}
\end{array}\right)\left(\begin{array}{c}
\mathrm{d} x \\
\mathrm{~d} y \\
\mathrm{~d} z \\
\mathrm{~d} \tilde{\alpha} \\
\mathrm{d} \tilde{\beta} \\
\mathrm{d} \tilde{\gamma}
\end{array}\right)
\end{aligned}
$$

where the $3 \times 3$-zero matrix is denoted by $\mathbf{0}$ and where the $3 \times 3$-matrices $M_{\beta, \gamma}, \tilde{M}_{\tilde{\beta}, \tilde{\alpha}}$ are given by

$\begin{aligned} M_{\beta, \alpha} & =\left(\begin{array}{ccc}0 & \sin \alpha & -\cos \alpha \sin \beta \\ 0 & \cos \alpha & \sin \alpha \sin \beta \\ 1 & 0 & \cos \beta\end{array}\right), \\ \tilde{M}_{\tilde{\beta}, \tilde{\alpha}} & =\left(\begin{array}{ccc}-\cos \tilde{\alpha} \tan \tilde{\beta} & \sin \tilde{\alpha} & \frac{\cos \tilde{\alpha}}{\cos \tilde{\beta}} \\ \sin \tilde{\alpha} \tan \tilde{\beta} & \cos \tilde{\alpha} & -\frac{\sin \tilde{\alpha}}{\cos \tilde{\beta}} \\ 1 & 0 & 0\end{array}\right)^{-T} .\end{aligned}$

Finally, we note that by linearity the $i$-th dual vector filters out the $i$-th component of a vector field $\sum_{j=1}^{6} v^{j} \mathcal{A}_{j}$

$\left\langle\mathrm{d} \mathcal{A}^{i}, \sum_{j=1}^{6} v^{j} \mathcal{A}_{j}\right\rangle=v^{i}, \quad$ for all $i, j=1, \ldots, 6$

\section{Left-Invariant Diffusions on $S E(3)=\mathbb{R}^{3} \rtimes S O(3)$ and $\mathbb{R}^{3} \rtimes S^{2}$}

In order to apply our general theory on diffusions on Lie groups, (Duits and Burgeth 2007), to suitable (convection-) diffusions on HARDI images, we naturally extend all functions $U: \mathbb{R}^{3} \rtimes S^{2} \rightarrow \mathbb{R}^{+}$to functions $\tilde{U}: \mathbb{R}^{3} \rtimes S O(3) \rightarrow \mathbb{R}^{+}$ by

$\tilde{U}(\mathbf{x}, R)=U\left(\mathbf{x}, R \mathbf{e}_{z}\right) \quad$ or in Euler angles:

$\tilde{U}\left(\mathbf{x}, R_{\mathbf{e}_{z}, \gamma} R_{\mathbf{e}_{y}, \beta} R_{\mathbf{e}_{z}, \alpha}\right)=U(\mathbf{x}, \mathbf{n}(\beta, \gamma))$.

Definition 4 We will call $\tilde{U}: \mathbb{R}^{3} \rtimes S O(3) \rightarrow \mathbb{R}$, given by (27), the HARDI-orientation score corresponding to HARDI image $U: \mathbb{R}^{3} \rtimes S^{2} \rightarrow \mathbb{R}$.

Here we note that the function $\tilde{U}$ in general is not equal to the wavelet transform of some image $f: \mathbb{R}^{d} \rightarrow \mathbb{R}$, in contrast to our previous works on invertible orientations of $2 \mathrm{D}$ images (Franken 2008; van Almsick 2005; Duits and van Almsick 2008; Duits and Franken 2010) and invertible orientation scores of 3D images (Duits et al. 2007).

We follow our general construction of scale space representations $\tilde{W}$ of functions $\tilde{U}$ defined on Lie groups (Duits and Burgeth 2007), where we consider the special case $S E(3)=\mathbb{R}^{3} \rtimes S O(3)$ :

$\left\{\begin{array}{l}\partial_{t} \tilde{W}(g, t)=Q^{\mathbf{D}, \mathbf{a}}\left(\mathcal{A}_{1}, \mathcal{A}_{2}, \ldots, \mathcal{A}_{6}\right) \tilde{W}(g, t), \\ \lim _{t \downarrow 0} \tilde{W}(g, t)=\tilde{U}(g)\end{array}\right.$ 
which is generated by a quadratic form on the left-invariant vector fields:

$$
Q^{\mathbf{D}, \mathbf{a}}\left(\mathcal{A}_{1}, \mathcal{A}_{2}, \ldots, \mathcal{A}_{6}\right)=\sum_{i=1}^{6}-a_{i} \mathcal{A}_{i}+\sum_{i, j=1}^{6} \mathcal{A}_{i} D_{i j} \mathcal{A}_{j}
$$

Now the Hörmander requirement, (Hormander 1968), on the symmetric $\mathbf{D}=\left[D_{i j}\right] \in \mathbb{R}^{6 \times 6}, \mathbf{D} \geq 0$ and $\mathbf{a}$, which guarantees smooth non-singular scale spaces for $S E(3)$, tells us that D need not be strictly positive definite. The Hörmander requirement is that all included generators together with their commutators should span the full tangent space. To this end for diagonal $\mathbf{D}$ one should consider the set

$\mathcal{S}=\left\{i \in\{1, \ldots, 6\} \mid D_{i i} \neq 0 \vee a_{i} \neq 0\right\}$,

now if for Example 1 is not in here then 3 and 5 must be in $\mathcal{S}$, or if 4 is not in $\mathcal{S}$ then 5 and 6 should be in $\mathcal{S}$. Following the general theory (Duits and Burgeth 2007) we note that iff the Hörmander condition is satisfied the solutions of the linear diffusions (i.e. D, a are constant) are given by $S E(3)$-convolution with a smooth probability kernel $\tilde{p}_{t}^{\mathbf{D}, \mathbf{a}}: \operatorname{SE}(3) \rightarrow \mathbb{R}^{+}$such that

$$
\begin{aligned}
& \tilde{W}(g, t)=\left(\tilde{p}_{t}^{\mathbf{D}, \mathbf{a}} *_{S E(3)} \tilde{U}\right)(g) \\
& \quad=\int_{S E(3)} \tilde{p}_{t}^{\mathbf{D}, \mathbf{a}}\left(h^{-1} g\right) \tilde{U}(h) \mathrm{d} \mu_{S E(3)}(h),
\end{aligned}
$$

$\lim _{t \downarrow 0} \tilde{p}_{t}^{\mathbf{D}, \mathbf{a}} *_{S E(3)} \tilde{U}=\tilde{U}$,

$$
\text { with } \tilde{p}_{t}^{\mathbf{D}, \mathbf{a}}>0 \quad \text { and } \quad \int_{S E(3)} \tilde{p}_{t}^{\mathbf{D}, \mathbf{a}}(g) \mathrm{d} \mu_{S E(3)}(g)=1,
$$

where the limit is taken in $\mathbb{L}_{2}(S E(3))$-sense.

The left-invariant diffusions on the group $S E(3)$ also give rise to left-invariant scale spaces on the homogeneous space $\mathbb{R}^{3} \rtimes S^{2} \equiv S E(3) /(\{\mathbf{0}\} \times S O(2))$ within the group. There are however, two important issues to be taken into account:

1. If we apply the diffusions directly to HARDI-orientation scores we can as well delete the last direction in our diffusions because clearly $\mathcal{A}_{6}=\partial_{\alpha}$ vanishes on functions which are not dependent on $\alpha$, i.e. $\partial_{\alpha} \tilde{U}=0$.

2. In order to naturally relate the (convection-)diffusions on HARDI-orientation scores, to (convection-)diffusions on HARDI images we have to make sure that the evolution equations are well defined on the cosets $S O(3) / S O(2)$, meaning that they do not depend on the choice of representant in the classes.

Next we formalize the second condition on diffusions on HARDI-orientation scores more explicitly. A movement along the equivalence classes $S O(3) / S O(2)$ is done by right multiplication with the subgroup $\operatorname{Stab}\left(\mathbf{e}_{z}\right) \equiv S O(2)$, with $\operatorname{Stab}\left(\mathbf{e}_{z}\right)=\left\{A \in S O(3) \mid A \mathbf{e}_{z}=\mathbf{e}_{z}\right\}$. Therefore our diffusion operator $\Phi_{t}$ which is the transform that maps the HARDIorientation score $\tilde{U}: \mathbb{R}^{3} \rtimes S O(3) \rightarrow \mathbb{R}^{+}$to a diffused HARDI-orientation score $\Phi_{t}(\tilde{U})=e^{t Q^{\mathbf{D}, \mathbf{a}}} \tilde{U}$, with stopping time $t>0$, should satisfy

$\left(\Phi_{t} \circ \mathcal{R}_{h}\right)(\tilde{U})=\Phi_{t}(\tilde{U})=\Phi_{t}\left(\mathcal{R}_{h} \tilde{U}\right)$

for all $h \in \operatorname{Stab}\left(\mathbf{e}_{z}\right) \equiv S O(2)$, where $\mathcal{R}_{h} \tilde{U}(g)=\tilde{U}(g h)$. Now (30) is satisfied iff

$\mathcal{R}_{\left(\mathbf{0}, R_{\mathbf{e}_{z}, \alpha}\right)} \circ Q^{\mathbf{D}, \mathbf{a}}\left(\mathcal{A}_{1}, \ldots, \mathcal{A}_{6}\right)=Q^{\mathbf{D}, \mathbf{a}}\left(\mathcal{A}_{1}, \ldots, \mathcal{A}_{6}\right)$.

Note that (30) and (31) are equivalent to

$\left(Q^{\mathbf{D}, \mathbf{a}}(\underline{\mathcal{A}}) \tilde{W}(\cdot, \cdot, t)\right)(g)=\left(Q^{\mathbf{D}, \mathbf{a}}(\underline{\mathcal{A}}) \tilde{W}(\cdot, \cdot, t)\right)(g h)$

for all $g \in S E(3), t>0, h=\left(\mathbf{0}, R_{\mathbf{e}_{z}, \alpha}\right)$ where $\underline{\mathcal{A}}=$ $\left(\mathcal{A}_{1}, \ldots, \mathcal{A}_{6}\right)^{T}$ and observe that $\underline{\mathcal{A}}_{g h} \tilde{U}=Z_{\alpha} \underline{\mathcal{A}}_{g} \tilde{U}$ with

$Z_{\alpha}=\left(\begin{array}{ccc|ccc}\cos \alpha & -\sin \alpha & 0 & 0 & 0 & 0 \\ \sin \alpha & \cos \alpha & 0 & 0 & 0 & 0 \\ 0 & 0 & 1 & 0 & 0 & 0 \\ \hline 0 & 0 & 0 & \cos \alpha & -\sin \alpha & 0 \\ 0 & 0 & 0 & \sin \alpha & \cos \alpha & 0 \\ 0 & 0 & 0 & 0 & 0 & 1\end{array}\right)$
$=R_{\mathbf{e}_{z}, \alpha} \oplus R_{\mathbf{e}_{z}, \alpha}, \quad Z_{\alpha} \in S O(6), R_{\mathbf{e}_{z}, \alpha} \in S O(3)$.

Hence for constant $\mathbf{D}$ and a (i.e. linear diffusion on the HARDI data) the requirement (31) simply reads

$$
\begin{aligned}
Q^{\mathbf{D}, \mathbf{a}}(\underline{\mathcal{A}}) & =Q^{\mathbf{D}, \mathbf{a}}\left(Z_{\alpha} \underline{\mathcal{A}}\right)=Q^{\left(Z_{\alpha}\right)^{T} \mathbf{D} Z_{\alpha}, Z_{\alpha} \mathbf{a}}(\underline{\mathcal{A}}) \\
\Leftrightarrow & \mathbf{a}=Z_{\alpha} \mathbf{a} \quad \text { and } \quad \mathbf{D}=Z_{\alpha} \mathbf{D} Z_{\alpha}^{T}
\end{aligned}
$$

which by Schur's lemma is the case if

$a^{1}=a^{2}=a^{4}=a^{5}=a^{6}=0 \quad$ and

$\mathbf{D}=\operatorname{diag}\left\{D_{11}, D_{11}, D_{33}, D_{44}, D_{44}, D_{66}=0\right\}$.

Analogously, for adaptive nonlinear diffusions, that is $\mathbf{D}$ and a not constant but depending on the initial condition $\tilde{U}$, i.e. $\mathbf{D}(\tilde{U}): S E(3) \rightarrow \mathbb{R}^{6 \times 6}$, with $(\mathbf{D}(\tilde{U}))^{T}=\mathbf{D}(\tilde{U})>0$ and $\mathbf{a}(\tilde{U})$ the requirement (31) simply reads

$\mathbf{a}(\tilde{U})(g h)=Z_{\alpha}^{T}(\mathbf{a}(\tilde{U}))(g) \quad$ and

$\mathbf{D}(\tilde{U})(g h)=Z_{\alpha} \mathbf{D}(\tilde{U})(g) Z_{\alpha}^{T}$.

for all $g \in S E(3), h=\left(\mathbf{0}, R_{\mathbf{e}_{z}, \alpha}\right)$. Summarizing all these results we conclude on HARDI data whose domain equals the homogeneous space $\mathbb{R}^{3} \rtimes S^{2}$ one has the following scale space representations:

$$
\begin{aligned}
& \partial_{t} W(\mathbf{y}, \mathbf{n}, t)=Q^{\mathbf{D}(U), \mathbf{a}(U)}\left(\mathcal{A}_{1}, \mathcal{A}_{2}, \ldots, \mathcal{A}_{5}, \mathcal{A}_{6}\right) W(\mathbf{y}, \mathbf{n}, t), \\
& W(\mathbf{y}, \mathbf{n}, 0)=U(\mathbf{y}, \mathbf{n})
\end{aligned}
$$


with $^{5} Q^{\mathbf{D}(U), \mathbf{a}(U)}\left(\mathcal{A}_{1}, \mathcal{A}_{2}, \ldots, \mathcal{A}_{5}, \mathcal{A}_{6}\right)=\sum_{i=1}^{5}\left(-a_{i} \mathcal{A}_{i}+\right.$ $\left.\sum_{j=1}^{5} \mathcal{A}_{i} D_{i j}(U) \mathcal{A}_{j}\right)$, where from now on we assume that $\mathbf{D}(U)$ and $\mathbf{a}(U)$ satisfy (35). In the linear case where $\mathbf{D}(U)=\mathbf{D}, \mathbf{a}(U)=\mathbf{a}$ this means that we shall automatically assume (34). In this case the solutions of (36) are given by the following kernel operators on $\mathbb{R}^{3} \rtimes S^{2}$ :

$$
\begin{aligned}
& W(\mathbf{y}, \mathbf{n}, t) \\
& =\left(p_{t}^{\mathbf{D}, \mathbf{a}} *_{\mathbb{R}^{3} \rtimes S^{2}} U\right)(\mathbf{y}, \mathbf{n}) \\
& =\int_{0}^{\pi} \int_{0}^{2 \pi} \int_{\mathbb{R}^{3}} p_{t}^{\mathbf{D}, \mathbf{a}}\left(\left(R_{\mathbf{e}_{z}, \gamma^{\prime}} R_{\mathbf{e}_{y}, \beta^{\prime}}\right)^{T}\left(\mathbf{y}-\mathbf{y}^{\prime}\right),\right. \\
& \left.\left.\quad\left(R_{\mathbf{e}_{z}, \gamma^{\prime}} R_{\mathbf{e}_{y}, \beta^{\prime}}\right)^{T} \mathbf{n}\right)\right) \\
& \cdot U\left(\mathbf{y}^{\prime}, \mathbf{n}\left(\beta^{\prime}, \gamma^{\prime}\right)\right) \mathrm{d} \mathbf{y}^{\prime} \mathrm{d} \sigma\left(\mathbf{n}\left(\beta^{\prime}, \gamma^{\prime}\right)\right),
\end{aligned}
$$

where the surface measure on the sphere is given by $\mathrm{d} \sigma\left(\mathbf{n}\left(\beta^{\prime}, \gamma^{\prime}\right)\right)=\sin \beta^{\prime} \mathrm{d} \gamma^{\prime} \mathrm{d} \beta^{\prime} \equiv \mathrm{d} \sigma(\tilde{\mathbf{n}}(\tilde{\beta}, \tilde{\gamma}))=$ $|\cos \tilde{\beta}| \mathrm{d} \tilde{\beta} \mathrm{d} \tilde{\gamma}$. Now in particular in the linear case, since $\left(\mathbb{R}^{3}, I\right)$ and $(\mathbf{0}, S O(3))$ are subgroups of $S E(3)$, we obtain the Laplace-Beltrami operators on these subgroups by means of:

$$
\begin{aligned}
\Delta_{S^{2}} & =Q^{\mathbf{D}=\operatorname{diag}\{0,0,0,1,1,1\}, \mathbf{a}=\mathbf{0}}(\underline{\mathcal{A}}) \\
& =\left(\mathcal{A}_{4}\right)^{2}+\left(\mathcal{A}_{5}\right)^{2}+\left(\mathcal{A}_{6}\right)^{2} \\
& =\left(\partial_{\beta}\right)^{2}+\cot (\beta) \partial_{\beta}+\sin ^{-2}(\beta)\left(\partial_{\gamma}\right)^{2}, \\
\Delta_{\mathbb{R}^{3}} & =Q^{\mathbf{D}=\operatorname{diag}\{1,1,1,0,0,0\}, \mathbf{a}=\mathbf{0}}(\underline{\mathcal{A})} \\
& =\left(\mathcal{A}_{1}\right)^{2}+\left(\mathcal{A}_{2}\right)^{2}+\left(\mathcal{A}_{3}\right)^{2} \\
& =\left(\partial_{x}\right)^{2}+\left(\partial_{y}\right)^{2}+\left(\partial_{z}\right)^{2} .
\end{aligned}
$$

Remark Recall that in the linear case we assumed (34) to ensure (31) so that (30) holds. It is not difficult to show, (Franken 2008, p. 170), that this implies the required symmetry (9) on the convolution kernel.

\subsection{Special Cases of Linear Left-invariant Diffusion on $\mathbb{R}^{3} \rtimes S^{2}$}

If we consider the singular case $\mathbf{D}=\operatorname{diag}\{1,1,1,0,0,0\}$, $\mathbf{a}=\mathbf{0}$ (not satisfying the Hörmander condition) we get the usual scale space in the position part only

$$
\begin{aligned}
W(\mathbf{y}, \mathbf{n}, t) & =\left(e^{t \Delta} U(\cdot, \mathbf{n})\right)(\mathbf{y}) \\
& =\mathcal{F}_{\mathbb{R}^{3}}^{-1}\left[\omega \mapsto \frac{e^{-t\|\omega\|^{2}}}{(2 \pi)^{\frac{3}{2}}} \mathcal{F}_{\mathbb{R}^{3}} f(\boldsymbol{\omega})\right](\mathbf{y}) \\
& =\left(G_{t} * f\right)(\mathbf{y}), \quad \text { with } G_{t}(\mathbf{y})=(4 \pi t)^{-\frac{3}{2}} e^{-\frac{\|\mathbf{y}\|^{2}}{4 t}}
\end{aligned}
$$

${ }^{5}$ Since $\mathcal{A}_{6} W(\mathbf{y}, \mathbf{n}, t)=0$ we set $a_{6}=D_{i 6}=0$ for $i=1, \ldots, 6$. Note that $\Delta_{L B} W(\mathbf{y}, \mathbf{n}, t)=\left(\left(\left(\mathcal{A}_{4}\right)^{2}+\left(\mathcal{A}_{5}\right)^{2}\right) W\right)(\mathbf{y}, \mathbf{n}, t)$. and consequently on $\mathbb{R}^{3} \rtimes S^{2}$ we have the singular distributional kernel $p_{t}^{\mathbf{D}, \mathbf{a}}(\mathbf{y}, \mathbf{n})=G_{t}(\mathbf{y}) \delta_{\mathbf{e}_{z}}(\mathbf{n})$, in (37).

If we consider the singular case $\mathbf{D}=\operatorname{diag}\{0,0,0,1,1,1\}$, $\mathbf{a}=\mathbf{0}$ we get the usual scale space on the sphere:

$W(\mathbf{y}, \mathbf{n}(\beta, \gamma), t)=\left(e^{t \Delta} S^{2} U(\mathbf{y}, \cdot)(\mathbf{x})\right.$

$$
\begin{aligned}
& =e^{t \Delta_{S}} \sum_{l=0}^{\infty} \sum_{m=-l}^{l}\left(Y_{l m}, U\right) Y_{l m}(\beta, \gamma) \\
& =\sum_{l=0}^{\infty} \sum_{m=-l}^{l}\left(Y_{l m}, U\right) e^{t \Delta^{2}} Y_{l m}(\beta, \gamma) \\
& =\sum_{l=0}^{\infty} \sum_{m=-l}^{l}\left(Y_{l m}, U\right) e^{-t(l(l+1))} Y_{l m}(\beta, \gamma) .
\end{aligned}
$$

where we note that the well-known spherical harmonics $\left\{Y_{l m}\right\}_{l=0, \ldots, \infty}^{m=-l, \ldots, l}$ form an orthonormal basis of $\mathbb{L}_{2}\left(S^{2}\right)$ and $\Delta_{S^{2}} Y_{l m}=-l(l+1) Y_{l m}$. Recall

$$
\begin{aligned}
Y_{l}^{m}(\beta, \gamma) & =\sqrt{\frac{(2 l+1)(l-|m|) !}{4 \pi(l+|m|) !}} P_{l}^{m}(\cos \beta) e^{i m \gamma} \\
l \in \mathbb{N}, m & =-l, \ldots, l .
\end{aligned}
$$

Consequently, on $\mathbb{R}^{3} \rtimes S^{2}$ we have the singular distributional kernel $p_{t}^{\mathbf{D}, \mathbf{a}}(\mathbf{y}, \mathbf{n})=g_{t}(\mathbf{n}) \delta_{\mathbf{0}}(\mathbf{y})$, in (37), where

$$
\begin{aligned}
g_{t}(\mathbf{n}(\beta, \gamma)) & =\sum_{l=0}^{\infty} \overline{Y_{l m}(\beta, \gamma)} Y_{l m}(\beta, \gamma) e^{-t l(l+1)} \\
& =\sum_{l=0}^{\infty}\left(P_{l}^{m}(\cos \beta)\right)^{2} \frac{(2 l+1)(l-|m|) !}{4 \pi(l+|m|) !} e^{-t l(l+1)} .
\end{aligned}
$$

Note that in the two cases mentioned above diffusion takes place either only along the spatial part or only along the angular part, which is not desirable as one wants to include line-models which exploit a natural coupling between position and orientation. Such a coupling is naturally included in a smooth way as long as the Hormander's condition is satisfied. In the two previous examples, the Hörmander condition is violated since both the span of $\left\{\mathcal{A}_{1}, \mathcal{A}_{2}, \mathcal{A}_{3}\right\}$ and the span of $\left\{\mathcal{A}_{4}, \mathcal{A}_{5}, \mathcal{A}_{6}\right\}$ are closed Lie-algebras, i.e. all commutators are again contained in the same 3-dimensional subspace of the 6-dimensional tangent space. Therefore we will consider more elaborate left-invariant convection, diffusions on $S E(3)$ with natural coupling between position and orientation. To explain what we mean with natural coupling we shall need the next definitions.

Definition 5 A curve $\gamma: \mathbb{R}^{+} \rightarrow \mathbb{R}^{3} \rtimes S^{2}$ given by $s \mapsto$ $\gamma(s)=(\mathbf{y}(s), \mathbf{n}(s))$ is called horizontal if $\mathbf{n}(s)=$ $\|\dot{\mathbf{y}}(s)\|^{-1} \dot{\mathbf{y}}(s)$. A tangent vector to a horizontal curve is called a horizontal tangent vector. A vector field $\mathcal{A}$ on 
$\mathbb{R}^{3} \rtimes S^{2}$ is horizontal if for all $(\mathbf{y}, \mathbf{n}) \in \mathbb{R}^{3} \rtimes S^{2}$ the tangent vector $\mathcal{A}_{(\mathbf{y}, \mathbf{n})}$ is horizontal. The horizontal part $\mathcal{H}_{g}$ of each tangent space is the vector-subspace of $T_{g}(S E(3))$ consisting of horizontal vector fields. Horizontal diffusion is diffusion which only takes place along horizontal curves.

It is not difficult to see that the horizontal part $\mathcal{H}_{g}$ of each tangent space $T_{g}(\operatorname{SE}(3))$ is spanned by $\left\{\mathcal{A}_{3}, \mathcal{A}_{4}, \mathcal{A}_{5}\right\}$. So all horizontal left-invariant convection diffusions are given by (36) where one must set $a_{1}=a_{2}=a_{6}=0, D_{j 2}=D_{2 j}=$ $D_{1 j}=D_{j 1}=D_{j 6}=D_{6 j}=0$ for all $j=1,2, \ldots, 6$. Now on a commutative group like $\mathbb{R}^{6}$ with commutative Liealgebra $\left\{\partial_{x_{1}}, \ldots, \partial_{x_{6}}\right\}$ omitting 3-directions (say $\partial_{x_{1}}, \partial_{x_{2}}$, $\partial_{x_{6}}$ ) from each tangent space in the diffusion would yield no smoothing along the global $x_{1}, x_{2}, x_{6}$-axes. In $S E(3)$ it is different since the commutators take care of indirect smoothing in the omitted directions $\left\{\mathcal{A}_{1}, \mathcal{A}_{2}, \mathcal{A}_{6}\right\}$, since

$$
\begin{aligned}
\operatorname{span}\left\{\mathcal{A}_{3}, \mathcal{A}_{4}, \mathcal{A}_{5},\left[\mathcal{A}_{3}, \mathcal{A}_{5}\right]=\mathcal{A}_{2},\right. \\
\left.\quad\left[\mathcal{A}_{4}, \mathcal{A}_{5}\right]=\mathcal{A}_{6},\left[\mathcal{A}_{5}, \mathcal{A}_{3}\right]=\mathcal{A}_{1}\right\}=T(\operatorname{SE}(3)) .
\end{aligned}
$$

Consider for example the $S E(3)$-analogues of the ForwardKolmogorov (or Fokker-Planck) equations of the direction process for contour-completion and the stochastic process for contour enhancement which we considered in our previous works, (Duits and Franken 2010), on $S E$ (2). Here we shall first provide the resulting PDEs and explain the underlying stochastic processes later in Sect. 4.2. The FokkerPlanck equation for (horizontal) contour completion on $S E(3)$ is given by

$$
\left\{\begin{array}{l}
\partial_{t} W(\mathbf{y}, \mathbf{n}, t) \\
\quad=\left(-\mathcal{A}_{3}+D\left(\left(\mathcal{A}_{4}\right)^{2}+\left(\mathcal{A}_{5}\right)^{2}\right)\right) W(\mathbf{y}, \mathbf{n}, t) \\
\quad=\left(-\mathcal{A}_{3}+D \Delta_{S^{2}}\right) W(\mathbf{y}, \mathbf{n}, t), \quad D=\frac{1}{2} \sigma^{2}>0, \\
\lim _{t \downarrow 0} W(\mathbf{y}, \mathbf{n}, t)=U(\mathbf{y}, \mathbf{n}),
\end{array}\right.
$$

where we note that $\left(\mathcal{A}_{6}\right)^{2}(W(\mathbf{y}, \mathbf{n}(\beta, \gamma), s))=0$. This equation arises from (36) by setting $D_{44}=D_{55}=D$ and $a_{3}=1$ and all other parameters to zero. The Fokker-Planck equation for (horizontal) contour enhancement is

$$
\left\{\begin{array}{l}
\partial_{t} W(\mathbf{y}, \mathbf{n}, t) \\
\quad=\left(D_{33}\left(\mathcal{A}_{3}\right)^{2}+D_{44}\left(\left(\mathcal{A}_{4}\right)^{2}+\left(\mathcal{A}_{5}\right)^{2}\right)\right) W(\mathbf{y}, \mathbf{n}, t) \\
\quad=\left(D_{33}\left(\mathcal{A}_{3}\right)^{2}+D \Delta_{S^{2}}\right) W(\mathbf{y}, \mathbf{n}, t), \\
\lim _{t \downarrow 0} W(\mathbf{y}, \mathbf{n}, t)=U(\mathbf{y}, \mathbf{n}) .
\end{array}\right.
$$

The solutions of the left-invariant diffusions on $\mathbb{R}^{3} \rtimes S^{2}$ given by (36) (with in particular (39) and (40)) are again given by convolution product (37) with a probability kernel $p_{t}^{\mathbf{D}, \mathbf{a}}$ on $\mathbb{R}^{3} \rtimes S^{2}$.
4.2 Brownian Motions on $S E(3)=\mathbb{R}^{3} \rtimes S O(3)$ and on $\mathbb{R}^{3} \rtimes S^{2}$

Next we formulate a left-invariant discrete Brownian motion on $S E$ (3) (expressed in the moving frame of reference). The left-invariant vector fields $\left\{\mathcal{A}_{1}, \ldots, \mathcal{A}_{6}\right\}$ form a moving frame of reference to the group. Here we note that there are two ways of considering vector fields. Either one considers them as differential operators on smooth locally defined functions, or one considers them as tangent vectors to equivalent classes of curves. These two viewpoints are equivalent, for formal proof see Aubin (2001, Prop. 2.4). Throughout this article we mainly use the first way of considering vector fields, but in this section we prefer to use the second way. We will write $\left\{\mathbf{e}_{1}(g), \ldots, \mathbf{e}_{6}(g)\right\}$ for the left-invariant vector fields (as tangent vectors to equivalence classes of curves) rather than the differential operators $\left\{\left.\mathcal{A}_{1}\right|_{g}, \ldots,\left.\mathcal{A}_{6}\right|_{g}\right\}$. We obtain the tangent vector $\mathbf{e}_{i}$ from $\mathcal{A}_{i}$ by replacing

$\partial_{x} \leftrightarrow(1,0,0,0,0,0)$,

$\partial_{y} \leftrightarrow(0,1,0,0,0,0)$,

$\partial_{z} \leftrightarrow(0,0,1,0,0,0)$,

$\partial_{\beta} \leftrightarrow(0,0,0, \alpha \cos \beta \cos \gamma, \alpha \cos \beta \sin \gamma,-\alpha \sin \beta)$,

$\partial_{\gamma} \leftrightarrow(0,0,0, \alpha \cos \gamma, \alpha \sin \gamma, 0)$,

$\partial_{\alpha} \leftrightarrow(0,0,0, \cos \gamma \sin \beta, \sin \gamma \sin \beta, \cos \beta)$,

where we identified $S O(3)$ with a ball with radius $2 \pi$ whose outer-sphere is identified with the origin, using Euler angles $R_{\mathbf{e}_{z}, \gamma} R_{\mathbf{e}_{y}, \beta} R_{\mathbf{e}_{z}, \alpha} \leftrightarrow \alpha \mathbf{n}(\beta, \gamma) \in B_{\mathbf{0}, 2 \pi}$. Next we formulate left-invariant discrete random walks on $S E(3)$ expressed in the moving frame of reference $\left\{\mathbf{e}_{i}\right\}_{i=1}^{6}$ given by (24) and (41):

$$
\begin{aligned}
\left(\mathbf{Y}_{n+1}, \mathbf{N}_{n+1}\right)= & \left(\mathbf{Y}_{n}, \mathbf{N}_{n}\right)+\left.\Delta s \sum_{i=1}^{5} a_{i} \mathbf{e}_{i}\right|_{\left(\mathbf{Y}_{n}, \mathbf{N}_{n}\right)} \\
& +\left.\sqrt{\Delta s} \sum_{i=1}^{5} \varepsilon_{i, n+1} \sum_{j=1}^{5} \sigma_{j i} \mathbf{e}_{j}\right|_{\left(\mathbf{Y}_{n}, \mathbf{N}_{n}\right)}
\end{aligned}
$$

for all $n=0, \ldots, N-1$,

$\left(\mathbf{Y}_{0}, \mathbf{N}_{0}\right) \sim U^{D}$,

with random variable $\left(\mathbf{Y}_{0}, \mathbf{N}_{0}\right)$ is distributed by $U^{D}$, where $U^{D}$ are the discretely sampled HARDI data (equidistant sampling in position and second order tessellation of the sphere) and where the random variables $\left(\mathbf{Y}_{n}, \mathbf{N}_{n}\right)$ are recursively determined using the independently normally distributed random variables $\left\{\varepsilon_{i, n+1}\right\}_{i=1, \ldots, 5}^{n=0, \ldots, N-1}, \varepsilon_{i, n+1} \sim \mathcal{N}(0,1)$ and with stepsize $\Delta s=\frac{s}{N}$ and where $\mathbf{a}:=\sum_{i=1}^{5} a_{i} \mathbf{e}_{i}$ denotes an a priori spatial velocity vector having constant coefficients $a_{i}$ with respect to the moving frame of reference 
$\left\{\mathbf{e}_{i}\right\}_{i=1}^{5}$ (just like in (29)). Now if we apply recursion and let $N \rightarrow \infty$ we get the following continuous Brownian motion processes on $S E(3)$ :

$$
\begin{aligned}
Y(t)= & Y(0)+\int_{0}^{t}\left(\left.\sum_{i=1}^{3} a_{i} \mathbf{e}_{i}\right|_{(Y(\tau), N(\tau))}\right. \\
& \left.+\left.\frac{1}{2} \tau^{-\frac{1}{2}} \varepsilon_{i} \sum_{j=1}^{3} \sigma_{j i} \mathbf{e}_{j}\right|_{(Y(\tau), N(\tau))}\right) \mathrm{d} \tau, \\
N(t)= & N(0)+\int_{0}^{t}\left(\left.\sum_{i=4}^{5} a_{i} \mathbf{e}_{i}\right|_{(Y(\tau), N(\tau))}\right. \\
& \left.+\left.\frac{1}{2} \tau^{-\frac{1}{2}} \varepsilon_{i} \sum_{j=4}^{5} \sigma_{j i} \mathbf{e}_{j}\right|_{(Y(\tau), N(\tau))}\right) \mathrm{d} \tau,
\end{aligned}
$$

with $\varepsilon_{i} \sim \mathcal{N}(0,1)$ and $(X(0), N(0)) \sim U$ and where $\sigma=$ $\sqrt{2 \mathbf{D}} \in \mathbb{R}^{6 \times 6}, \sigma>0$. Note that $\mathrm{d} \sqrt{\tau}=\frac{1}{2} \tau^{-\frac{1}{2}} \mathrm{~d} \tau$.

Now if we set $U=\delta_{\mathbf{0}, \mathbf{e}_{z}}$ (i.e. at time zero ) then suitable averaging of infinitely many random walks of this process yields the transition probability $(\mathbf{y}, \mathbf{n}) \mapsto p_{t}^{\mathbf{D}, \mathbf{a}}(\mathbf{y}, \mathbf{n})$ which is the Green's function of the left-invariant evolution equations (36) on $\mathbb{R}^{3} \rtimes S^{2}$. In general the PDEs (36) are the Forward Kolmogorov equation of the general stochastic process (42). This follows by Ito-calculus and in particular Ito's formula for formulas on a stochastic process, see Chirikjian and Kyatkin (2001) and see van Almsick (2005, App. A) where one should consistently replace the left-invariant vector fields of $\mathbb{R}^{n}$ by the left-invariant vector fields on $\mathbb{R}^{3} \rtimes S^{2}$.

In particular we have now formulated the direction process for contour completion in $\mathbb{R}^{3} \rtimes S^{2}$ (i.e. non-zero parameters in (42) are $D_{44}=D_{55}>0, a_{3}>0$ with FokkerPlanck equation given by (39)), and the (horizontal) Brownian motion for contour-enhancement in $\mathbb{R}^{3} \rtimes S^{2}$ (i.e. nonzero parameters in (42) are $D_{33}>0, D_{44}=D_{55}>0$ with Fokker-Planck equation given by (40)).

\subsection{Tikhonov-Regularization of HARDI Images}

In the previous subsection we have formulated the Brownian-motions (42) underlying all linear left-invariant convection-diffusion equations on HARDI data, with in particular the direction process for contour completion and (horizontal) Brownian motion for contour-enhancement. However, we only considered the time dependent stochastic processes and as mentioned before in Markov-processes traveling time is memoryless and thereby negatively exponentially distributed $T \sim N E(\lambda)$, i.e. $P(T=t)=\lambda e^{-\lambda t}$ with expectation $E(T)=\lambda^{-1}$, for some $\lambda>0$. Recall our observations (12) and (13) and thereby by means of Laplacetransform with respect to time we relate the Fokker-Planck equations to their resolvent equations, as at least formally we have

$$
\begin{aligned}
W(\mathbf{y}, \mathbf{n}, t) & =\left(e^{t\left(Q^{\mathbf{D}, \mathbf{a}}(\underline{\mathcal{A}})\right)} U\right)(\mathbf{y}, \mathbf{n}) \quad \text { and } \\
P_{\gamma}(\mathbf{y}, \mathbf{n}, t) & =\lambda \int_{0}^{\infty} e^{-t \lambda}\left(e^{t\left(Q^{\mathbf{D}, \mathbf{a}}(\underline{\mathcal{A}})\right)} U\right)(\mathbf{y}, \mathbf{n}) \mathrm{d} t \\
& =\lambda\left(\lambda I-Q^{\mathbf{D}, \mathbf{a}}(\underline{\mathcal{A}})\right)^{-1} U(\mathbf{y}, \mathbf{n}),
\end{aligned}
$$

for $t, \lambda>0$ and all $\mathbf{y} \in \mathbb{R}^{3}, \mathbf{n} \in S^{2}$, where the negative definite generator $Q^{\mathbf{D}, \mathbf{a}}$ is given by (29) and again with $\underline{\mathcal{A}} U=\left(\mathcal{A}_{1} U, \ldots, \mathcal{A}_{6} U\right)$. This is similar to our introductory example on the torus in Sect. 3.2. The resolvent operator $\lambda\left(\lambda I-Q^{\mathbf{D}=\operatorname{diag}\left(D_{i i}\right), \mathbf{a}=0}(\underline{\mathcal{A}})\right)^{-1}$ occurs in a first order Tikhonov regularization as we show in the next theorem.

Theorem 2 Let $U \in \mathbb{L}_{2}\left(\mathbb{R}^{3} \rtimes S^{2}\right)$ and $\lambda, D_{33}>0, D_{44}=$ $D_{55}>0, D_{11}=D_{22}>0$. Then the unique solution of the variational problem

$$
\begin{aligned}
& \underset{P \in \mathbb{H}^{1}\left(\mathbb{R}^{3} \rtimes S^{2}\right)}{\arg \min } \int_{\mathbb{R}^{3} \rtimes S^{2}} \frac{\lambda}{2}(P(\mathbf{y}, \mathbf{n})-U(\mathbf{y}, \mathbf{n}))^{2} \\
& +\sum_{k=1}^{5} D_{k k}\left|\mathcal{A}_{k} P(\mathbf{y}, \mathbf{n})\right|^{2} \mathrm{~d} \mathbf{y d} \sigma(\mathbf{n})
\end{aligned}
$$

is given by $P_{U}^{\lambda}(\mathbf{y}, \mathbf{n})=\left(R_{\lambda}^{\mathbf{D}} *_{\mathbb{R}^{3} \rtimes S^{2}} U\right)(\mathbf{y}, \mathbf{n})$, where the Green's function $R_{\lambda}^{\mathbf{D}}: \mathbb{R}^{3} \rtimes S^{2} \rightarrow \mathbb{R}^{+}$is the Laplacetransform of the heat-kernel with respect to time: $R_{\lambda}^{\mathbf{D}}(\mathbf{y}, \mathbf{n})=$ $\lambda \int_{0}^{\infty} p_{t}^{\mathbf{D}, \mathbf{a}=\mathbf{0}}(\mathbf{y}, \mathbf{n}) e^{-t \lambda} \mathrm{d} t$ with $\mathbf{D}=\operatorname{diag}\left\{D_{11}, \ldots, D_{55}, 0\right\}$. $P_{U}^{\lambda}(\mathbf{y}, \mathbf{n})$ equals the probability of finding a random walker in $\mathbb{R}^{3} \rtimes S^{2}$ regardless its traveling time at position $\mathbf{y} \in \mathbb{R}^{3}$ with orientation $\mathbf{n} \in S^{2}$ starting from initial distribution $U$ at time $t=0$.

For a proof we refer to our technical report (Duits and Franken 2009, Chap. 4.3).

\section{Differential Geometry: The Underlying Cartan-Connection on $S E(3)$ and the Auto-Parallels in $S E(3)$}

Now that we have constructed all left-invariant scale space representations on HARDI images, generated by means of a quadratic form (29) on the left-invariant vector fields on $S E(3)$. The question rises what is the underlying differential geometry for these evolutions?

For example, as the left-invariant vector fields clearly vary per position in the group yielding a moving frame of reference attached to luminosity particles (random walkers in $\mathbb{R}^{3} \rtimes S^{2}$ embedded in $S E(3)$ ) with both a position and an orientation, the question rises along which trajectories in 
$\mathbb{R}^{3} \rtimes S^{2}$ do these particles move? Furthermore, as the leftinvariant vector fields are obtained by the push-forward of the left-multiplication on the group,

$\mathcal{A}_{g}=\left(L_{g}\right)_{*} \mathcal{A}_{e}, \quad$ i.e. $\quad \mathcal{A}_{g} \tilde{\phi}=\mathcal{A}_{e}\left(\tilde{\phi} \circ L_{g}\right)$,

where $L_{g} h=g h, g, h \in S E(3), \tilde{\phi}: S E(3) \rightarrow \mathbb{R}$ smooth,

the question rises whether this defines a connection between all tangent spaces, such that these trajectories are autoparallel with respect to this connection? Finally, we need a connection to rigid body mechanics described in a moving frame of reference, to get some physical intuition in the choice of the fundamental constants ${ }^{6}\left\{a_{i}\right\}_{i=1}^{6}$ and $\left\{D_{i j}\right\}_{i, j=1}^{6}$ within our generators (29).

In order to get some first physical intuition on analysis and differential geometry along the moving frame $\left\{\mathcal{A}_{1}, \ldots, \mathcal{A}_{6}\right\}$ and its dual frame $\left\{\mathrm{d} \mathcal{A}^{1}, \ldots, \mathrm{d} \mathcal{A}^{6}\right\}$, we will make some preliminary remarks on the well-known theory of rigid body movements described in moving coordinate systems. Imagine a curve in $\mathbb{R}^{3}$ described in the moving frame of reference (embedded in the spatial part of the group $S E(3)$ ), describing a rigid body movement with constant spatial velocity $\hat{\mathbf{c}}^{(1)}$ and constant angular velocity $\hat{\mathbf{c}}^{(2)}$ and parameterized by arc-length $s>0$. Suppose the curve is given by

$\mathbf{y}(s)=\left.\sum_{i=1}^{3} \alpha^{i}(s) \mathcal{A}_{i}\right|_{\mathbf{y}(s)} \quad$ where $\alpha^{i} \in C^{2}([0, L], \mathbb{R})$,

such that $\hat{\mathbf{c}}^{(1)}=\left.\sum_{i=1}^{3} \dot{\alpha}^{i}(s) \mathcal{A}_{i}\right|_{\mathbf{y}(s)}$ for all $s>0$. Now if we differentiate twice with respect to the arc-length parameter and keep in mind that $\left.\frac{d}{d s} \mathcal{A}_{i}\right|_{\mathbf{y}(s)}=\hat{\mathbf{c}}^{(2)} \times\left.\mathcal{A}_{i}\right|_{\mathbf{y}(s)}$, we get

$\ddot{\mathbf{y}}(s)=0+2 \hat{\mathbf{c}}^{(2)} \times \hat{\mathbf{c}}^{(1)}+\hat{\mathbf{c}}^{(2)} \times\left(\hat{\mathbf{c}}^{(2)} \times \mathbf{y}(s)\right)$.

In words: The absolute acceleration equals the relative acceleration (which is zero, since $\hat{\mathbf{c}}^{(1)}$ is constant) plus the Coriolis acceleration $2 \hat{\mathbf{c}}^{(2)} \times \hat{\mathbf{c}}^{(1)}$ and the centrifugal acceleration $\hat{\mathbf{c}}^{(2)} \times\left(\hat{\mathbf{c}}^{(2)} \times \mathbf{y}(s)\right)$. Now in case of uniform circular motion the speed is constant but the velocity is always tangent to the orbit of acceleration and the acceleration has constant magnitude and always points to the center of rotation. In this case, the total sum of Coriolis acceleration and centrifugal acceleration add up to the well-known centripetal acceleration,

$$
\begin{aligned}
\ddot{\mathbf{y}}(s) & =2 \hat{\mathbf{c}}^{(2)} \times\left(-\hat{\mathbf{c}}^{(2)} \times R \mathbf{r}(s)\right)+\hat{\mathbf{c}}^{2} \times\left(\hat{\mathbf{c}}^{(2)} \times R \mathbf{r}(s)\right) \\
& =-\left\|\hat{\mathbf{c}}^{(2)}\right\|^{2} R \mathbf{r}(s)=-\frac{\left\|\hat{\mathbf{c}}^{1}\right\|^{2}}{R} \mathbf{r}(s),
\end{aligned}
$$

\footnotetext{
${ }^{6}$ Or later in Sect. 9 to get some intuition in the choice of functions $\left\{a_{i}\right\}_{i=1}^{6}$ and $\left\{D_{i j}\right\}_{i, j=1}^{6}$.
}

where $R$ is the radius of the circular orbit $\mathbf{y}(s)=\mathbf{m}+R \mathbf{r}(s)$, $\|\mathbf{r}(s)\|=1$. The centripetal acceleration equals half the Coriolis acceleration, i.e. $\ddot{\mathbf{y}}(s)=\hat{\mathbf{c}}^{(2)} \times \hat{\mathbf{c}}^{(1)}$.

In our previous work (Duits and Franken 2010, Part II) on contour-enhancement and completion via left-invariant diffusions on invertible orientation scores (complex-valued functions on $S E(2)$ ) we put a lot of emphasis on the underlying differential geometry in $S E(2)$. All results straightforwardly generalize to the case of HARDI images, which can be considered as functions on $\mathbb{R}^{3} \rtimes S^{2}$ embedded in $S E(3)$. These rather technical results are summarized in Theorem 3 , which answers all questions raised in the beginning of this section. Unfortunately, this theorem requires general differential geometrical concepts such as principal fiber bundles, associated vector bundles, tangent bundles, framebundles and the Cartan-Ehresmann connection defined on them. These concepts are explained in full detail in Spivak (1975) (with a very nice overview on p. 386).

The reader who is not familiar with these technicalities from differential geometry can skip the first part of the theorem while accepting the formula of the covariant derivatives given in (48), where the anti-symmetric Christoffel symbols are equal to minus the structure constants $c_{i j}^{k}=-c_{j i}^{k}$ (recall (22)) of the Lie-algebra. Here we stress that we follow the Cartan viewpoint on differential geometry, where connections are expressed in moving coordinate frames (we use the frame of left-invariant vector fields $\left\{\mathcal{A}_{1}, \ldots, \mathcal{A}_{6}\right\}$ derived in Sect. 3.3 for this purpose) and thereby we have nonvanishing torsion. ${ }^{7}$ This is different from the Levi-Civita connection for differential geometry on Riemannian manifolds, which is much more common in image analysis. The Levi-Civita connection is the unique torsion free metric compatible connection on a Riemannian manifold and because of this vanishing torsion of the Levi-Civita connection $\bar{\nabla}$ there is a 1-to-1 relation ${ }^{8}$ to the Christoffel symbols (required for covariant derivatives $\bar{\nabla}_{i} v^{j}=\partial_{i} v^{j}+\Gamma_{i j}^{k} \partial_{k} v^{j}$ ) and the derivatives of the metric tensor. In the more general Cartan connection outlined below, however, one can have non-vanishing torsion and the Christoffels are not necessarily related to a metric tensor, nor need they be symmetric.

Theorem 3 The Maurer-Cartan form $\omega$ on SE(3) is given by

$\omega_{g}\left(X_{g}\right)=\sum_{i=1}^{6}\left\langle\left.\mathrm{~d} \mathcal{A}^{i}\right|_{g}, X_{g}\right\rangle A_{i}, \quad X_{g} \in T_{g}(S E(3))$,

\footnotetext{
${ }^{7}$ The torsion tensor $T_{\nabla}$ of a connection $\nabla$ is given by $T_{\nabla}[X, Y]=$ $\nabla_{X} Y-\nabla_{Y} X-[X, Y]$. The torsion-tensor $T_{\bar{\nabla}}$ of a Levi-Civita connection vanishes, whereas the torsion-tensor of our Cartan connection $\nabla$ on $S E(3)$ is given by $T_{\nabla}=3 \sum_{i, j, k=1}^{6} c_{i j}^{k} \mathrm{~d} \mathcal{A}^{i} \otimes \mathrm{d} \mathcal{A}^{j} \otimes \mathcal{A}_{k}$.

${ }^{8}$ In a Levi-Civita connection one has $\Gamma_{k l}^{i}=\Gamma_{l k}^{i}=\frac{1}{2} \sum_{m} g^{i m}\left(g_{m k, l}+\right.$ $\left.g_{m l, k}-g_{k l, m}\right)$ with respect to a holonomic basis.
} 
where the dual vectors $\left\{\mathrm{d} \mathcal{A}^{i}\right\}_{i=1}^{6}$ are given by (26) and $A_{i}=\left.\mathcal{A}_{i}\right|_{e}$. It is a Cartan Ehresmann connection form on the principal fiber bundle $P=(\operatorname{SE}(3), e, S E(3), \mathcal{L}(\operatorname{SE}(3)))$, where $\pi(g)=e, R_{g} u=u g, u, g \in S E(3)$. Let Ad denote the adjoint action of $S E(3)$ on its own Lie-algebra $T_{e}(S E(3))$, i.e. $A d(g)=\left(R_{g^{-1}} L_{g}\right)_{*}$, i.e. the push-forward of conjugation. Then the adjoint representation of $S E(3)$ on the vector space $\mathcal{L}(S E(3))$ of left-invariant vector fields is given by

$\widetilde{A d}(g)=\mathrm{d} \mathcal{R} \circ A d(g) \circ \omega$.

This adjoint representation gives rise to the associated vector bundle $S E(3) \times \widetilde{A d} \mathcal{L}(S E(3))$. The corresponding connection form on this vector bundle is given by

$\tilde{\omega}=\sum_{j=1}^{6} \tilde{a d}\left(\mathcal{A}_{j}\right) \otimes \mathrm{d} \mathcal{A}^{j}=\sum_{i, j, k=1}^{6} c_{i j}^{k} \mathcal{A}_{k} \otimes \mathrm{d} \mathcal{A}^{i} \otimes \mathrm{d} \mathcal{A}^{j}$,

with $\tilde{a d}=(\widetilde{A d})_{*}$, i.e. $\tilde{a d}\left(\mathcal{A}_{j}\right)=\sum_{i=1}^{6}\left[\mathcal{A}_{i}, \mathcal{A}_{j}\right] \otimes \mathrm{d} \mathcal{A}^{i}$ (Jost $2005, p$. 265). Then $\tilde{\omega}$ yields the following $6 \times 6$-matrix valued 1 -form

$\tilde{\omega}_{j}^{k}(\cdot):=-\tilde{\omega}\left(\mathrm{d} \mathcal{A}^{k}, \cdot, \mathcal{A}_{j}\right) \quad k, j=1,2, \ldots, 6$,

on the frame bundle, (Spivak 1975, p. 353, p. 359), where the sections are moving frames (Spivak 1975, p. 354). Let $\left\{\mu_{k}\right\}_{k=1}^{6}$ denote the sections in the tangent bundle $E:=$ (SE(3), T(SE(3))) which coincide with the left-invariant vector fields $\left\{\mathcal{A}_{k}\right\}_{k=1}^{6}$. Then the matrix-valued 1 -form given by (47) yields the Cartan connection given by the covariant derivatives

$$
\begin{aligned}
& D_{\left.X\right|_{\gamma(t)}}(\mu(\gamma(t))):=D \mu(\gamma(t))\left(\left.X\right|_{\gamma(t)}\right) \\
& =\sum_{k=1}^{6} \dot{a}^{k}(t) \mu_{k}(\gamma(t)) \\
& \quad+\sum_{k=1}^{6} a^{k}(\gamma(t)) \sum_{j=1}^{6} \tilde{\omega}_{k}^{j}\left(\left.X\right|_{\gamma(t)}\right) \mu_{j}(\gamma(t)) \\
& =\sum_{k=1}^{6} \dot{a}^{k}(t) \mu_{k}(\gamma(t))+\sum_{i, j, k=1}^{6} \dot{\gamma}^{i}(t) a^{k}(\gamma(t)) \Gamma_{i k}^{j} \mu_{j}(\gamma(t))
\end{aligned}
$$

with $\dot{a}^{k}(t)=\sum_{i=1}^{6} \dot{\gamma}^{i}(t)\left(\left.\mathcal{A}_{i}\right|_{\gamma(t)} a^{k}\right)$, for all tangent vectors $\left.X\right|_{\gamma(t)}=\left.\sum_{i=1}^{6} \dot{\gamma}^{i}(t) \mathcal{A}_{i}\right|_{\gamma(t)}$ along a curve $t \mapsto \gamma(t) \in$ SE(2) and all sections $\mu(\gamma(t))=\sum_{k=1}^{6} a^{k}(\gamma(t)) \mu_{k}(\gamma(t))$. The Christoffel symbols in (48) are constant $\Gamma_{i k}^{j}=-c_{i k}^{j}$, with $c_{i k}^{j}$ the structure constants of Lie-algebra $T_{e}(S E(3))$. Consequently, the connection D has constant curvature and constant torsion and the left-invariant evolution equations given in (28) can be rewritten in covariant derivatives (using short notation $\nabla_{j}:=D_{\mathcal{A}_{j}}$ ):

$$
\left\{\begin{aligned}
\partial_{t} W(g, t)= & \sum_{i=1}^{6}-a^{i}(W) \mathcal{A}_{i} W(g, t) \\
& +\sum_{i, j=1}^{6} \mathcal{A}_{i}\left(\left(D_{i j}(W)\right)(g, t) \mathcal{A}_{j} W\right)(g, t) \\
= & \sum_{i=1}^{6}-a^{i}(W) \nabla_{i} W(g, t) \\
& +\sum_{i, j=1}^{6} \nabla_{i}\left(\left(D_{i j}(W)\right)(g, t) \nabla_{j} W\right)(g, t)
\end{aligned}\right.
$$$$
W(g, 0)=\tilde{U}(g), \quad \text { for all } g \in \operatorname{SE}(3), t>0 \text {. }
$$

Both convection and diffusion in the left-invariant evolution equations (28) take place along the exponential curves $\gamma_{\mathbf{c}, g}(t)=g \cdot e^{t \sum_{i=1}^{6} c^{i} A_{i}}$ in $S E(3)$ which are the covariantly constant curves (i.e. auto-parallels) with respect to the Cartan connection. In particular, if $a^{i}(W)=c^{i}$ constant and if $D_{i j}(W)=0$ (convection case) then the solutions are

$W(g, t)=\tilde{U}\left(g \cdot e^{-t \sum_{i=1}^{6} c^{i} A_{i}}\right)$.

The spatial projections $\mathbb{P}_{\mathbb{R}^{3}} \gamma$ of these of the auto-parallel/ exponential curves $\gamma$ are circular spirals with constant curvature and constant torsion. The curvature magnitude equals $\left\|\hat{\mathbf{c}}^{(1)}\right\|^{-1}\left\|\hat{\mathbf{c}}^{(2)} \times \hat{\mathbf{c}}^{(1)}\right\|$ and the curvature vector equals

$$
\begin{aligned}
\boldsymbol{\kappa}(t)= & \frac{1}{\left\|\hat{\mathbf{c}}^{(1)}\right\|}\left(\cos \left(t\left\|\hat{\mathbf{c}}^{(2)}\right\|\right) \hat{\mathbf{c}}^{(2)} \times \hat{\mathbf{c}}^{(1)}\right. \\
& \left.+\frac{\sin \left(t\left\|\hat{\mathbf{c}}^{(2)}\right\|\right)}{\left\|\hat{\mathbf{c}}^{(2)}\right\|} \hat{\mathbf{c}}^{(2)} \times \hat{\mathbf{c}}^{(2)} \times \hat{\mathbf{c}}^{(1)}\right),
\end{aligned}
$$

where $\mathbf{c}=\left(c^{1}, c^{2}, c^{3} ; c^{4}, c^{5}, c^{6}\right)=\left(\hat{\mathbf{c}}^{(1)} ; \hat{\mathbf{c}}^{(2)}\right)$. The torsion vector equals $\boldsymbol{\tau}(t)=\left|\hat{\mathbf{c}}_{1} \cdot \hat{\mathbf{c}}_{2}\right| \boldsymbol{\kappa}(t)$.

Proof The proof is a straightforward generalization from our previous results (Duits and Franken 2010, Part II, Thm. 3.8 and Thm. 3.9) on the $S E(2)$-case to the case $S E(3)$. The formulas of the constant torsion and curvature of the spatial part of the auto-parallel curves (which are the exponential curves) follow by the formula (54) for (the spatial part $\mathbf{x}(s)$ of) the exponential curves, which we will derive in Sect. 5.1. Here we stress that $s(t)=$ $t \sqrt{\left(c^{1}\right)^{2}+\left(c^{2}\right)^{2}+\left(c^{3}\right)^{2}}$ is the arc-length of the spatial part of the exponential curve and where we recall that $\boldsymbol{\kappa}(s)=\ddot{\mathbf{x}}(s)$ and $\boldsymbol{\tau}(s)=\frac{d}{d s}(\dot{\mathbf{x}}(s) \times \ddot{\mathbf{x}}(s))$. Note that both the formula (54) for the exponential curves in the next section 
and the formulas for torsion and curvature are simplifications of our earlier formulas (Franken 2008, pp. 175-177). In the special case of only convection the solution (50) follows by $e^{t \mathrm{~d} \mathcal{R}(A)} \tilde{U}(g)=\mathcal{R}_{e^{t A}} \tilde{U}(g)$, with $A=-\sum_{i=1}^{6} c^{i} A_{i}$ and $\mathrm{d} \mathcal{R}(A)=-\sum_{i=1}^{6} c^{i} \mathcal{A}_{i}$ with $\mathcal{A}_{i}=\mathrm{d} \mathcal{R}\left(A_{i}\right)$.

\subsection{The Exponential Curves and the Logarithmic Map} Explicitly in Euler Angles

Next we compute the exponential curves in $S E(3)$ by an isomorphism of the group $S E(3)$ to matrix group $\mathfrak{S E}(3)$

$$
\operatorname{SE}(3) \ni\left(\mathbf{x}, R_{\gamma, \beta, \alpha}\right) \leftrightarrow\left(\begin{array}{cc}
R_{\gamma, \beta, \alpha} \mathbf{x} \\
0 & 1
\end{array}\right) \in \mathfrak{S E}(3)
$$$$
\text { with } R_{\gamma, \beta, \alpha}=R_{\mathbf{e}_{z}, \gamma} R_{\mathbf{e}_{y}, \beta} R_{\mathbf{e}_{z}, \alpha} \text {. }
$$

This isomorphism induces the following isomorphism between the respective Lie-algebras

$$
\begin{aligned}
\sum_{i=1}^{6} c^{i} \mathcal{A}_{i} & \in \mathcal{L}(\operatorname{SE}(3)) \leftrightarrow T_{e}(\operatorname{SE}(3)) \\
& \ni \sum_{i=1}^{6} c^{i} A_{i} \leftrightarrow \sum_{i=1}^{6} c^{i} X_{i} \in \mathbb{R}^{4 \times 4},
\end{aligned}
$$

where $\left\{c^{i}\right\}_{i=1}^{6} \in \mathbb{R}^{6}$ and with matrices $\left\{X_{i}\right\}_{i=1}^{6} \in \mathbb{R}^{4 \times 4}$ are given by

$$
\begin{array}{rlrl}
X_{1} & =\left(\begin{array}{cccc}
0 & 0 & 0 & 1 \\
0 & 0 & 0 & 0 \\
0 & 0 & 0 & 0 \\
0 & 0 & 0 & 0
\end{array}\right), & X_{2}=\left(\begin{array}{llll}
0 & 0 & 0 & 0 \\
0 & 0 & 0 & 1 \\
0 & 0 & 0 & 0 \\
0 & 0 & 0 & 0
\end{array}\right), \\
X_{3}=\left(\begin{array}{cccc}
0 & 0 & 0 & 0 \\
0 & 0 & 0 & 0 \\
0 & 0 & 0 & 1 \\
0 & 0 & 0 & 0
\end{array}\right), & X_{4}=\left(\begin{array}{cccc}
0 & 0 & 0 & 0 \\
0 & 0 & -1 & 0 \\
0 & 1 & 0 & 0 \\
0 & 0 & 0 & 0
\end{array}\right), \\
X_{5}=\left(\begin{array}{cccc}
0 & 0 & 1 & 0 \\
0 & 0 & 0 & 0 \\
-1 & 0 & 0 & 0 \\
0 & 0 & 0 & 0
\end{array}\right), & X_{6}=\left(\begin{array}{cccc}
0 & -1 & 0 & 0 \\
1 & 0 & 0 & 0 \\
0 & 0 & 0 & 0 \\
0 & 0 & 0 & 0
\end{array}\right) .
\end{array}
$$

Note that $\mathcal{A}_{i} \leftrightarrow A_{i} \leftrightarrow X_{i} \Rightarrow\left[A_{i}, A_{j}\right] \leftrightarrow\left[\mathcal{A}_{i}, \mathcal{A}_{j}\right] \leftrightarrow$ $\left[X_{i}, X_{j}\right]$ and indeed direct computation yields:

$$
\begin{aligned}
& \sum_{k=1}^{6} c_{i j}^{k} \mathcal{A}_{k}=\left[\mathcal{A}_{i}, \mathcal{A}_{j}\right] \\
& \quad \leftrightarrow\left[X_{i}, X_{j}\right]=\sum_{k=1}^{6} c_{i j}^{k} X_{k} \text { with commutator table }
\end{aligned}
$$

$$
\left(\begin{array}{cccccc}
0 & 0 & 0 & 0 & X_{3} & -X_{2} \\
0 & 0 & 0 & -X_{3} & 0 & X_{1} \\
0 & 0 & 0 & X_{2} & -X_{1} & 0 \\
0 & X_{3} & -X_{2} & 0 & X_{6} & -X_{5} \\
-X_{3} & 0 & X_{1} & -X_{6} & 0 & X_{4} \\
X_{2} & -X_{1} & 0 & X_{5} & -X_{4} & 0
\end{array}\right)
$$

where $i$ enumerates vertically and $j$ horizontally and $\left[\mathcal{A}_{i}, \mathcal{A}_{j}\right]=\mathcal{A}_{i} \mathcal{A}_{j}-\mathcal{A}_{j} \mathcal{A}_{i}$ and $\left[X_{i}, X_{j}\right]=X_{i} X_{j}-X_{j} X_{i}$. Each element in the Lie-algebra of the matrix group $\mathfrak{S E}(3)$ can be written

$$
\begin{aligned}
A & =\sum_{i=1}^{6} c^{i} X_{i}=\left(\begin{array}{cc}
\Omega & \hat{\mathbf{c}}^{(1)} \\
0 & 0
\end{array}\right), \\
\Omega & =\left(\begin{array}{ccc}
0 & -c^{6} & c^{5} \\
c^{6} & 0 & -c^{4} \\
-c^{5} & c^{4} & 0
\end{array}\right) \in \operatorname{so}(3),
\end{aligned}
$$

$\hat{\mathbf{c}}^{(2)}=\left(c^{1}, c^{2}, c^{3}\right) \in \mathbb{R}^{3}$,

with $\operatorname{so}(3)=\left\{A \in \mathbb{R}^{3 \times 3} \mid A^{T}=-A\right\}$. Note that $\exp (\operatorname{so}(3))=S O(3)$ and $\Omega \mathbf{x}=\hat{\mathbf{c}}^{(2)} \times \mathbf{x}$ and set $\tilde{q}=\left\|\hat{\mathbf{c}}^{(2)}\right\|=$ $\sqrt{\left(c^{4}\right)^{2}+\left(c^{5}\right)^{2}+\left(c^{6}\right)^{2}}$ so that $\Omega^{3}=-\tilde{q}^{2} \Omega$ and therefore

$$
\begin{aligned}
A^{k}=\left(\begin{array}{cc}
\Omega^{k} & \Omega^{k-1} \hat{\mathbf{c}}^{(1)} \\
0 & 0
\end{array}\right) \\
\Rightarrow \quad e^{t A}=\sum_{k=1}^{\infty} \frac{t^{k}}{k !} A^{k}=\left(\begin{array}{cc}
e^{t \Omega} & t\left(\int_{0}^{1} e^{t s \Omega} \mathrm{d} s\right) \hat{\mathbf{c}}^{(1)} \\
0 & 1
\end{array}\right) \\
=\left(\begin{array}{ll}
R & \mathbf{x} \\
0 & 1
\end{array}\right) \in \mathfrak{S E E}(3),
\end{aligned}
$$

with

$$
\begin{aligned}
\int_{0}^{1} e^{t s \Omega} \mathrm{d} s & =\sum_{k=0}^{\infty} \frac{(t \Omega)^{k}}{(k+1) !} \\
& =I+\frac{1}{t} \frac{1-\cos (\tilde{q} t)}{\tilde{q}^{2}} \Omega+\frac{\tilde{q}-t^{-1} \sin (\tilde{q} t)}{\tilde{q}^{3}} \Omega^{2},
\end{aligned}
$$

and

$$
R=e^{t \Omega}=I+\frac{\sin (\tilde{q} t)}{\tilde{q}} \Omega+\frac{1-\cos (\tilde{q} t)}{\tilde{q}^{2}} \Omega^{2}
$$

so that the exponential curves are given by

$$
\begin{aligned}
& \gamma_{\mathbf{c}}(t)=e^{t \sum_{i=1}^{6} c^{i} A_{i}} \\
& =\left\{\begin{array}{l}
\left(c_{1} t, c_{2} t, c_{3} t, I\right) \quad \text { if } \hat{\mathbf{c}}^{(2)}=\mathbf{0}, \\
\left(t \hat{\mathbf{c}}^{(1)}+\frac{1-\cos (\tilde{q} t)}{\tilde{q}^{2}} \Omega \hat{\mathbf{c}}^{(1)}+\left(t \tilde{q}^{-2}-\frac{\sin (\tilde{q} t)}{\tilde{q}^{3}}\right) \Omega^{2} \hat{\mathbf{c}}^{(1)},\right. \\
\left.I+\frac{\sin (\tilde{q} t)}{\tilde{q}} \Omega+\frac{(1-\cos (\tilde{q} t))}{\tilde{q}^{2}} \Omega^{2}\right) \quad \text { else. }
\end{array}\right.
\end{aligned}
$$


As the exponential map is surjective we are also interested in the logarithmic map. This means we have to solve for $\hat{\mathbf{c}}^{(1)} \in$ $\mathbb{R}^{3}$ and $\Omega \in \operatorname{so}(3)$, given a group element $g=\left(\mathbf{x}, R_{\gamma, \beta, \alpha}\right) \in$ $S E(3)$. Note that $\Omega^{T}=-\Omega,\left(\Omega^{2}\right)^{T}=\Omega^{2}$ so that $R-R^{T}=$ $2 \frac{\sin (\tilde{q} t)}{\tilde{q}} \Omega$ from which the logarithmic map $\Omega=\log _{S O(3)} R$, $R=R_{\gamma, \beta, \alpha}$ follows explicitly:

$c^{4}=c_{\gamma, \beta, \alpha}^{4}:=\frac{\tilde{q}}{2 \sin \tilde{q}} \sin \beta(\sin \alpha-\sin \gamma)$,

$c^{5}=c_{\gamma, \beta, \alpha}^{5}:=\frac{\tilde{q}}{2 \sin \tilde{q}} \sin \beta(\cos \alpha-\cos \gamma)$,

$c^{6}=c_{\gamma, \beta, \alpha}^{6}:=\frac{\tilde{q}}{2 \sin \tilde{q}}\left(2 \cos ^{2}\left(\frac{\beta}{2}\right) \sin (\alpha+\gamma)\right)$

and thereby $\tilde{q}=\sqrt{\left(c^{4}\right)^{2}+\left(c^{5}\right)^{2}+\left(c^{6}\right)^{2}}=\tilde{q}_{\gamma, \beta, \alpha}=$ $\arcsin \sqrt{\cos ^{2}\left(\frac{\alpha+\gamma}{2}\right) \sin ^{2} \beta+\cos ^{4}\left(\frac{\beta}{2}\right) \sin ^{2}(\alpha+\gamma)}$. So it remains to express $\hat{\mathbf{c}}^{(1)}=\left(c^{1}, c^{2}, c^{3}\right)^{T}$ in Euler angles $(\gamma, \beta, \alpha)$. Now $\Omega^{3}=-\tilde{q}^{2} \Omega$ implies that

$$
\begin{aligned}
&\left(I+\tilde{q}^{-2}(1-\cos \tilde{q}) \Omega+\tilde{q}^{-3}(\tilde{q}-\sin \tilde{q}) \Omega^{2}\right) \hat{\mathbf{c}}^{(1)}=\mathbf{x} \\
& \Leftrightarrow \quad \hat{\mathbf{c}}^{(1)}= \hat{\mathbf{c}}_{\mathbf{x}, \gamma, \beta, \alpha}^{(1)} \\
&:=\left(I-\frac{1}{2} \Omega_{\gamma, \beta, \alpha}+\tilde{q}_{\gamma, \beta, \alpha}^{-2}\left(1-\frac{\tilde{q}_{\gamma, \beta, \alpha}}{2}\right.\right. \\
&\left.\left.\cdot \cot \left(\frac{\tilde{q}_{\gamma, \beta, \alpha}}{2}\right)\right)\left(\Omega_{\gamma, \beta, \alpha}\right)^{2}\right) \mathbf{x} .
\end{aligned}
$$

Now equality (55) and (56) provide the explicit logarithmic mapping on $S E(3)$ :

$\log _{S E(3)}\left(\mathbf{x}, R_{\gamma, \beta, \alpha}\right)=\sum_{i=1}^{3} c_{\mathbf{x}, \gamma, \beta, \alpha}^{i} A_{i}+\sum_{i=4}^{6} c_{\gamma, \beta, \alpha}^{i} A_{i}$.

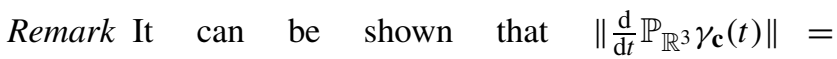
$\sqrt{\left(c^{1}\right)^{2}+\left(c^{2}\right)^{2}+\left(c^{3}\right)^{2}}$. Consequently the arc-length parameter $s>0$ is expressed in $t$ by means of $s(t)=$ $t \sqrt{\left(c^{1}\right)^{2}+\left(c^{2}\right)^{2}+\left(c^{3}\right)^{2}}$. If we want to impose spatial arc-length parameterizations of curves in $S E(3)$ we must rescale all $c_{i} \rightarrow \frac{c_{i}}{\sqrt{\left(c^{1}\right)^{2}+\left(c^{2}\right)^{2}+\left(c^{3}\right)^{2}}}$ so that $\left\|\hat{\mathbf{c}}^{(1)}\right\|=$ $\sqrt{\left(c^{1}\right)^{2}+\left(c^{2}\right)^{2}+\left(c^{3}\right)^{2}}=1$.

Remark The group $S E(3)$ is isomorphic to the group of rigid motions in $\mathbb{R}^{3}$ well-known in mechanics. The vector $\hat{\mathbf{c}}^{(1)}$ denotes constant velocity in the moving coordinate frame $\left\{\mathcal{A}_{i}\right\}_{i=1}^{3}$ whereas $\hat{\mathbf{c}}^{(2)}$ denotes constant angular velocity with respect to the same moving coordinate frame attached to a particle on a moving rigid body in $\mathbb{R}^{3}$. Note that $\kappa(0)$ equals the centripetal acceleration at the moving frame of reference $\left.\left\{\mathcal{A}_{1}, \mathcal{A}_{2}, \mathcal{A}_{3}\right\}\right|_{\gamma_{\mathbf{c}}(0)=e}=\left\{A_{1}, A_{2}, A_{3}\right\}$, whereas $\kappa(s)$ equals the centripetal acceleration at the moving frame of reference $\left.\left\{\mathcal{A}_{1}, \mathcal{A}_{2}, \mathcal{A}_{3}\right\}\right|_{\gamma_{\mathbf{c}}(s)}$, but again expressed in the global coordinate system $\left\{A_{1}, A_{2}, A_{3}\right\}=\left\{\partial_{x}, \partial_{y}, \partial_{z}\right\}$ of the spatial part $\equiv \mathbb{R}^{3}$ of the group, which is for $s>0$ no longer aligned with the moving frame of reference. To re-express $\kappa(s)$ in $\left\{A_{1}, A_{2}, A_{3}\right\}$ one must rotate $\kappa(0)$ over an angle of $s\left\|\hat{\mathbf{c}}^{(2)}\right\|$ around the angular velocity $\hat{\mathbf{c}}^{2}$, which explains (51).

\section{Analysis of the Convolution Kernels of Scale Spaces on HARDI Images}

It is a notorious problem to find explicit formulas for the exact Green's functions $p_{t}^{\mathbf{D}, \mathbf{a}}: \mathbb{R}^{3} \rtimes S^{2} \rightarrow \mathbb{R}^{+}$of the leftinvariant diffusions, (36), on $\mathbb{R}^{3} \rtimes S^{2}$. Explicit, tangible and exact formulas for heat-kernels on $S E(3)$ do not seem to exist in literature. Nevertheless, there does exist a nice general theory overlapping the fields of functional analysis and group theory, see for example (Nagel and Ricci 1990; ter Elst and Robinson 1998), which at least provides Gaussian estimates for Green's functions of left-invariant diffusions on Lie groups, generated by subcoercive operators. In the remainder of this section we will employ this general theory to our special case where $\mathbb{R}^{3} \rtimes S^{2}$ is embedded into $S E(3)$ and we will derive new explicit and useful approximation formulas for these Green's functions. Within this section we always use the second coordinate chart, (4), as it is highly preferable over the more common Euler angle parametrization, (1), since we rather avoid singularities at the unity element of $S E(3)$. We refer to Duits and Franken (2009, App. A) for an accurate approximation to the exact Green's functions for the direction process 39 (a contour-completion process) in $\mathbb{R}^{3} \rtimes S^{2}$, likewise (Duits and van Almsick 2008), where we managed to derive the exact Green's functions of the direction process in $S E(2)$. However, unlike the $S E(2)$-case, we do have to apply a reasonable approximation in the generator in order to get tangible approximation formulas. These approximations are valid for $4 t D_{44}$ small and are nearly exact in a sharp cone around the $z$-axis where the Green's function is concentrated.

We shall first carry out the method of contraction. This method typically relates the group of positions and rotations to a (nilpotent) group positions and velocities and serves as an essential pre-requisite for our Gaussian estimates and approximation kernels later on. The reader who is not so much interested in the detailed analysis can skip this section and continue with the numerics explained in Chap. 7.

\subsection{Local Approximation of $S E$ (3) by a Nilpotent Group via Contraction}

The group $S E(3)$ is not nilpotent. This makes it hard to get tangible explicit formulae for the heat-kernels. Therefore we shall generalize our Heisenberg approximations of the Green's functions on $S E(2)$, (Duits and van Almsick 2008; 
Thornber and Williams 2000; van Almsick 2005), to the case $S E(3)$. Again we will follow the general work by ter Elst and Robinson (1998) on semigroups on Lie groups generated by weighted subcoercive operators. In their general work we consider a particular case by setting the Hilbert space $\mathbb{L}_{2}(S E(3))$, the group $S E(3)$ and the right-regular representation $\mathcal{R}$. Furthermore we consider the algebraic basis $\left\{\mathcal{A}_{3}, \mathcal{A}_{4}, \mathcal{A}_{5}\right\}$ leading to the following filtration of the Lie algebra

$$
\begin{aligned}
\mathfrak{g}_{1} & =\operatorname{span}\left\{\mathcal{A}_{3}, \mathcal{A}_{4}, \mathcal{A}_{5}\right\} \\
& \subset \mathfrak{g}_{2}=\operatorname{span}\left\{\mathcal{A}_{1}, \mathcal{A}_{2}, \mathcal{A}_{3}, \mathcal{A}_{4}, \mathcal{A}_{5}, \mathcal{A}_{6}\right\}=\mathcal{L}(\operatorname{SE}(3)) .
\end{aligned}
$$

Now that we have this filtration we have to assign weights to the generators

$w_{3}=w_{4}=w_{5}=1 \quad$ and $\quad w_{1}=w_{2}=w_{6}=2$.

For example $w_{3}=1$ since $\mathcal{A}_{3}$ already occurs in $\mathfrak{g}_{1}, w_{6}=2$ since $\mathcal{A}_{6}$ is within in $\mathfrak{g}_{2}$ and not in $\mathfrak{g}_{1}$. Now that we have these weights we define the following dilations on the Liealgebra $T_{e}(\operatorname{SE}(3))\left(\right.$ recall $\left.A_{i}=\left.\mathcal{A}_{i}\right|_{e}\right)$ :

$$
\begin{aligned}
& \gamma_{q}\left(\sum_{i=1}^{6} c^{i} A_{i}\right)=\sum_{i=1}^{6} q^{w_{i}} c^{i} A_{i}, \quad \text { for all } c^{i} \in \mathbb{R}, \\
& \tilde{\gamma}_{q}\left(x, y, z, R_{\tilde{\gamma}, \tilde{\beta}, \tilde{\alpha}}\right) \\
& \quad=\left(\frac{x}{q^{w_{1}}}, \frac{y}{q^{w_{2}}}, \frac{z}{q^{w_{3}}}, R_{\frac{\tilde{\gamma}}{q^{w_{4}}}, \frac{\tilde{\beta}}{q^{w_{5}}}, \frac{\tilde{\alpha}}{q^{w_{6}}}}\right), \quad q>0,
\end{aligned}
$$

and for $0<q \leq 1$ we define the Lie product $[A, B]_{q}=$ $\gamma_{q}^{-1}\left[\gamma_{q}(A), \gamma_{q}(B)\right]$. Now let $(S E(3))_{q}$ be the simply connected Lie group generated by the Lie algebra $\left(T_{e}(S E(3))\right.$, $\left.[\cdot, \cdot]_{q}\right)$. This Lie group is isomorphic to the matrix group with group product:

$$
\begin{aligned}
& \left(\mathbf{x}, \tilde{R}_{\tilde{\gamma}, \tilde{\beta}, \tilde{\alpha}}\right) \cdot{ }_{q}\left(\mathbf{x}^{\prime}, \tilde{R}_{\tilde{\gamma}^{\prime}, \tilde{\beta}^{\prime}, \tilde{\alpha}^{\prime}}\right) \\
& \quad=\left(\mathbf{x}+S_{q} \cdot \tilde{R}_{\tilde{\gamma} q, \tilde{\beta} q, \tilde{\alpha} q^{2}} \cdot S_{q^{-1}} \mathbf{x}^{\prime}, \tilde{R}_{\tilde{\gamma}, \tilde{\beta}, \tilde{\alpha}} \cdot \tilde{R}_{\tilde{\gamma}^{\prime}, \tilde{\beta}^{\prime}, \tilde{\alpha}^{\prime}}\right)
\end{aligned}
$$

where $S_{q}:=\operatorname{diag}\{1,1, q\} \in \mathbb{R}^{3 \times 3}$ and we used short-notation $\tilde{R}_{\tilde{\gamma}, \tilde{\beta}, \tilde{\alpha}}=R_{\mathbf{e}_{x}, \tilde{\gamma}} R_{\mathbf{e}_{y}, \tilde{\beta}} R_{\mathbf{e}_{z}, \tilde{\alpha}}$, i.e. our elements of $S O(3)$ are expressed in the second coordinate chart, (4). Now the left-invariant vector fields on the group $(S E(3))_{q}$ are given by $\left.\mathcal{A}_{i}^{q}\right|_{g}=\left(\tilde{\gamma}_{q}^{-1} \circ L_{g} \circ \tilde{\gamma}_{q}\right)_{*} A_{i}, i=1, \ldots, 6$. Straightforward (but intense) calculations yield (for each $g=\left(\mathbf{x}, \tilde{R}_{\tilde{\gamma}, \tilde{\beta}, \tilde{\alpha}}\right) \in$ $\left.(S E(3))_{q}\right)$ :

$$
\begin{aligned}
\left.\mathcal{A}_{1}^{q}\right|_{g}= & \cos \left(q^{2} \tilde{\alpha}\right) \cos (q \tilde{\beta}) \partial_{x}+\left(\cos (\tilde{\gamma} q) \sin \left(\tilde{\alpha} q^{2}\right)\right. \\
& \left.+\cos \left(\tilde{\alpha} q^{2}\right) \sin (\tilde{\beta} q) \sin (\tilde{\gamma} q)\right) \partial_{y} \\
& +q\left(\sin \left(\tilde{\alpha} q^{2}\right) \sin (\tilde{\gamma} q)\right. \\
& \left.-\cos \left(\tilde{\alpha} q^{2}\right) \cos (\tilde{\gamma} q) \sin (\tilde{\beta} q)\right) \partial_{z}
\end{aligned}
$$

$$
\begin{aligned}
\left.\mathcal{A}_{2}^{q}\right|_{g}= & -\sin \left(\tilde{\alpha} q^{2}\right) \cos (\tilde{\beta} q) \partial_{x}+\left(\cos \left(q^{2} \tilde{\alpha}\right) \cos (\tilde{\gamma} q)\right. \\
& \left.-\sin \left(\tilde{\alpha} q^{2}\right) \sin (\tilde{\beta} q) \sin (\tilde{\gamma} q)\right) \partial_{y} \\
& +q\left(\sin \left(\tilde{\alpha} q^{2}\right) \sin (\tilde{\beta} q) \cos (\tilde{\beta} q)\right. \\
& \left.+\cos \left(\tilde{\alpha} q^{2}\right) \sin (\tilde{\gamma} q)\right) \partial_{z} \\
\left.\mathcal{A}_{3}^{q}\right|_{g}= & q^{-1} \sin (\tilde{\beta} q) \partial_{x}-q^{-1} \cos (\tilde{\beta} q) \sin (\tilde{\gamma} q) \partial_{y} \\
& +\cos (\tilde{\beta} q) \cos (\tilde{\gamma} q) \partial_{z} \\
\left.\mathcal{A}_{4}^{q}\right|_{g}= & -q^{-1} \cos \left(\tilde{\alpha} q^{2}\right) \tan (\tilde{\beta} q) \partial_{\tilde{\alpha}}+\sin (\tilde{\alpha} q) \partial_{\tilde{\beta}} \\
& +\frac{\cos \left(\tilde{\alpha} q^{2}\right)}{\cos (\tilde{\beta} q)} \partial_{\tilde{\gamma}} \\
\left.\mathcal{A}_{5}^{q}\right|_{g}= & q^{-1} \sin \left(\tilde{\alpha} q^{2}\right) \tan (\tilde{\beta} q) \partial_{\tilde{\alpha}}+\cos \left(\tilde{\alpha} q^{2}\right) \partial_{\tilde{\beta}} \\
& -\frac{\sin \left(q^{2} \tilde{\alpha}\right)}{\cos (q \tilde{\beta})} \partial_{\tilde{\gamma}} \\
\left.\mathcal{A}_{6}^{q}\right|_{g}= & \partial_{\tilde{\alpha}} \cdot
\end{aligned}
$$

Note that $\left[A_{i}, A_{j}\right]_{q}=\gamma_{q}^{-1}\left[\gamma_{q}\left(A_{i}\right), \gamma_{q}\left(A_{j}\right)\right]=$ $\gamma_{q}^{-1} q^{w_{i}+w_{j}}\left[A_{i}, A_{j}\right]=\sum_{k=1}^{6} q^{w_{i}+w_{j}-w_{k}} c_{i j}^{k} A_{k}$ and thereby

$\left[A_{4}, A_{5}\right]_{q}=A_{6}, \quad\left[A_{4}, A_{6}\right]_{q}=-q^{2} A_{5}$,

$\left[A_{5}, A_{6}\right]_{q}=q^{2} A_{4}, \quad\left[A_{4}, A_{3}\right]_{q}=-A_{2}$,

$\left[A_{4}, A_{2}\right]_{q}=q^{2} A_{3}, \quad\left[A_{5}, A_{1}\right]_{q}=-q^{2} A_{3}$,

$\left[A_{5}, A_{3}\right]_{q}=A_{1}, \quad\left[A_{6}, A_{1}\right]_{q}=q^{2} A_{2} \quad$ and

$\left[A_{6}, A_{2}\right]_{q}=-q^{2} A_{1}$.

Analogously to the case $q=1,(S E(3))_{q=1}=S E(3)$ we have an isomorphism of the common Lie-algebra at the unity element $T_{e}(S E(3))=T_{e}\left((S E(3))_{q}\right)$ and left-invariant vector fields on the group $(S E(3))_{q}$ :

$$
\left(A_{i} \leftrightarrow \mathcal{A}_{i}^{q} \text { and } A_{j} \leftrightarrow \mathcal{A}_{j}^{q}\right) \quad \Rightarrow \quad\left[A_{i}, A_{j}\right]_{q} \leftrightarrow\left[\mathcal{A}_{i}^{q}, \mathcal{A}_{j}^{q}\right]
$$

It can be verified that the left-invariant vector fields $\mathcal{A}_{i}^{q}$ satisfy the same commutation relations given in (61).

Now let us consider the case $q \downarrow 0$, then we get a nilpotent-group $(S E(3))_{0}$ with left-invariant vector fields

$\mathcal{A}_{1}^{0}=\partial_{x}, \quad \mathcal{A}_{2}^{0}=\partial_{y}, \quad \mathcal{A}_{3}^{0}=\tilde{\beta} \partial_{x}-\tilde{\gamma} \partial_{y}+\partial_{z}$,

$\mathcal{A}_{4}^{0}=-\tilde{\beta} \partial_{\tilde{\alpha}}+\partial_{\tilde{\gamma}}, \quad \mathcal{A}_{5}^{0}=\partial_{\tilde{\beta}}, \quad \mathcal{A}_{6}^{0}=\partial_{\tilde{\alpha}}$.

\subsubsection{The Heisenberg-approximation of the Contour Completion Kernel}

Recall that the generator of contour completion diffusion equals $-\mathcal{A}_{3}+D_{44}\left(\left(\mathcal{A}_{4}\right)^{2}+\left(\mathcal{A}_{5}\right)^{2}\right)$. So let us replace the true left-invariant vector fields $\left\{\mathcal{A}_{i}\right\}_{i=3}^{5}$ on $\operatorname{SE}(3)=(S E(3))_{q=1}$ 
by their Heisenberg-approximations $\left\{\mathcal{A}_{i}^{0}\right\}_{i=3}^{5}$ that are given

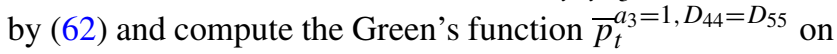
$(S E(3))_{0}$ (i.e. the convolution kernel which yields the solutions of contour completion on $(S E(3))_{0}$ by group convolution on $\left.(S E(3))_{0}\right)$. For $0<D_{44} \ll 1$ this kernel is a local approximation of the true contour completion kernel $p_{t}^{a_{3}=1, D_{44}=D_{55}}$, on $\mathbb{R}^{3} \rtimes S^{2}$ :

$$
\begin{aligned}
\bar{p}_{t}^{a_{3}=} & 1, D_{44}=D_{55} \\
:= & e^{t\left(-\mathcal{A}_{3}^{0}+D_{44}\left(\left(\mathcal{A}_{4}^{0}\right)^{2}+\left(\mathcal{A}_{5}^{0}\right)^{2}\right)\right)} \delta_{0}^{x} \otimes \delta_{0}^{y} \otimes \delta_{0}^{z} \otimes \delta_{0}^{\tilde{\gamma}} \otimes \delta_{0}^{\tilde{\beta}} \Rightarrow \\
\bar{p}_{t}^{a_{3}=} & 1, D_{44}=D_{55}(x, y, z, \tilde{\mathbf{n}}(\tilde{\beta}, \tilde{\gamma})) \\
= & \delta(t-z)\left(e^{t\left(-\tilde{\beta} \partial_{x}+D_{44}\left(\partial_{\tilde{\beta}}\right)^{2}\right)} \delta_{0}^{x} \otimes \delta_{0}^{\tilde{\beta}}\right)(x, \tilde{\beta}) \\
& \cdot\left(e^{t\left(\tilde{\gamma} \partial_{y}+D_{44}\left(\partial_{\tilde{\gamma}}\right)^{2}\right)} \delta_{0}^{y} \otimes \delta_{0}^{\tilde{\gamma}}\right)(y, \tilde{\gamma}) \\
= & \delta(t-z) \frac{3}{4\left(D_{44} \pi z^{2}\right)^{2}} e^{-\frac{12(x-(1 / 2) z \tilde{\beta})^{2}+z^{2} \tilde{\beta}^{2}}{4 z^{3} D_{44}}} \\
& \cdot e^{-\frac{12(y+(1 / 2) z \tilde{\gamma})^{2}+z^{2} \tilde{\gamma}^{2}}{4 z^{3} D_{44}}},
\end{aligned}
$$

where $\tilde{\mathbf{n}}(\tilde{\beta}, \tilde{\gamma})=R_{\mathbf{e}_{x}, \tilde{\gamma}} R_{\mathbf{e}_{y}, \tilde{\beta}} \mathbf{e}_{z}=(\sin \tilde{\beta},-\sin \tilde{\gamma} \cos \tilde{\beta}$, $\cos \tilde{\gamma} \cos \tilde{\beta})^{T}$. The corresponding resolvent kernel on the group $(S E(3))_{0}$ is now directly obtained by Laplace transform with respect to time

$$
\begin{aligned}
\bar{R}_{\lambda}^{a_{3}=} & =1, D_{44}=D_{55}(x, y, z, \tilde{\mathbf{n}}(\tilde{\beta}, \tilde{\gamma})) \\
= & \left\{\begin{array}{cc}
\frac{3}{4\left(D_{44} \pi z^{2}\right)^{2}} \lambda e^{-\lambda z} e^{-\frac{12(x-(1 / 2) z \tilde{\beta})^{2}+z^{2} \tilde{\beta}^{2}}{4 z^{3} D_{44}}} \\
\cdot e^{-\frac{12(y+(1 / 2) z \tilde{\gamma})^{2}+z^{2} \tilde{\gamma}^{2}}{4 z^{3} D_{44}}} & \text { if } z>0 \\
0 \quad & \text { if } z \leq 0 \text { and }(x, y) \neq(0,0) .
\end{array}\right.
\end{aligned}
$$

So we make a remarkable observation: The Heisenbergapproximation, (63), of the contour completion kernel in $(S E(3))$ is a direct product of two Heisenberg approximations of contour completion kernels in $S E(2)$, (Duits and van Almsick 2008),

$$
\begin{aligned}
& \bar{p}_{t}^{a_{3}, D_{44}=D_{55} ;(S E(3))_{0}}(x, y, z, \tilde{\mathbf{n}}(\tilde{\beta}, \tilde{\gamma})) \\
& \quad=\bar{p}_{t}^{a_{3}, D_{44} ;(S E(2))_{0}}(z, x, \tilde{\beta}) \cdot \bar{p}_{t}^{a_{3}, D_{44} ;(S E(2))_{0}}(z,-y, \tilde{\gamma}) .
\end{aligned}
$$

Now since the Heisenberg approximation kernel $\bar{p}_{t}^{D_{33}, D_{44} ;(S E(2))_{0}}$ is for reasonable parameter settings (that is $\left.0<\frac{D_{44}}{D_{33}} \ll 1\right)$ close to the exact kernel $\bar{p}_{t}^{D_{33}, D_{44} ;(\operatorname{SE}(2))}$ we heuristically propose for these reasonable parameter settings the same direct-product approximation for the exact contour-enhancement kernels on $\mathbb{R}^{3} \rtimes S^{2}$ :

$$
\begin{aligned}
& p_{t}^{a_{3}, D_{44}=D_{55} ; \mathbb{R}^{3} \rtimes S^{2}}(x, y, z, \tilde{\mathbf{n}}(\tilde{\beta}, \tilde{\gamma})) \\
& \quad \approx p_{t}^{a_{3}, D_{44} ; S E(2)}(z, x, \tilde{\beta}) \cdot p_{t}^{a_{3}, D_{44} ; S E(2)}(z,-y, \tilde{\gamma}),
\end{aligned}
$$

where the exact kernels $p_{t}^{a_{3}, D_{44} ; S E(2)}: S E(2) \rightarrow \mathbb{R}^{+}$for contour completion in $S E(2)$ can be found in Duits and van Almsick (2008).

\subsubsection{The Heisenberg-approximation of the Contour Enhancement Kernel}

Recall that the generator of contour completion diffusion equals $D_{33}\left(\mathcal{A}_{3}\right)^{2}+D_{44}\left(\left(\mathcal{A}_{4}\right)^{2}+\left(\mathcal{A}_{5}\right)^{2}\right)$. So let us replace the true left-invariant vector fields $\left\{\mathcal{A}_{i}\right\}_{i=3}^{5}$ on $S E(3)=(S E(3))_{q=1}$ by their Heisenberg-approximations $\left\{\mathcal{A}_{i}^{0}\right\}_{i=3}^{5}$ given by (62) and consider the Green's function $\bar{p}_{t}^{D_{33}, \bar{D}_{44}=D_{55}}$ on $(S E(3))_{0}$ :

$$
\begin{aligned}
\bar{p}_{t}^{a_{3}}=1, D_{44}=D_{55} \\
\quad:=e^{t\left(D_{33}\left(\mathcal{A}_{3}^{0}\right)^{2}+D_{44}\left(\left(\mathcal{A}_{4}^{0}\right)^{2}+\left(\mathcal{A}_{5}^{0}\right)^{2}\right)\right)} \delta_{0}^{x} \otimes \delta_{0}^{y} \otimes \delta_{0}^{z} \otimes \delta_{0}^{\tilde{\gamma}} \otimes \delta_{0}^{\tilde{\beta}},
\end{aligned}
$$

which is not easy to compute. However we follow the same approach as we applied previously (Duits and Franken 2010) to the diffusion kernels on the 2D Euclidean motion group $(S E(2))_{0}$, which follows the coordinate substitutions as proposed by Citti and Sarti (2006). The group $(S E(3))_{0}$ however is not (entirely) a direct product of two $\mathrm{H}_{3}$ groups and application of the coordinate-transformation

$x_{1}^{\prime}=x_{2}^{\prime}=\frac{z}{2 \sqrt{2 D_{44}}}, \quad \omega_{1}=\frac{\tilde{\beta}}{\sqrt{2 D_{44}}} \quad$ and

$\omega_{2}^{\prime}=\frac{\tilde{\gamma}}{\sqrt{2 D_{44}}}, \quad t_{1}^{\prime}=\frac{2\left(x-\frac{\tilde{\beta} z}{4}\right)}{\sqrt{D_{44} D_{33}}}, \quad t_{2}^{\prime}=\frac{-2\left(y+\frac{\tilde{\gamma} z}{4}\right)}{\sqrt{D_{33} D_{44}}}$,

expressed the generator into

$$
\begin{aligned}
&\left(D_{33}\left(\mathcal{A}_{3}^{0}\right)^{2}+D_{44}\left(\left(\mathcal{A}_{4}^{0}\right)^{2}+\left(\mathcal{A}_{5}^{0}\right)^{2}\right)\right) \\
&=\frac{1}{2}\left(\partial_{\omega_{1}^{\prime}}-2 x_{1}^{\prime} \partial_{t_{1}^{\prime}}\right)^{2}+\frac{1}{2}\left(\partial_{\omega_{2}^{\prime}}-2 x_{2}^{\prime} \partial_{t_{2}^{\prime}}\right)^{2} \\
& \quad+\frac{1}{2}\left(\partial_{x_{2}^{\prime}}+2 \omega_{2}^{\prime} \partial_{t_{2}^{\prime}}\right)^{2}+\frac{1}{2}\left(\partial_{x_{1}^{\prime}}+2 \omega_{1}^{\prime} \partial_{t_{1}^{\prime}}\right)^{2} \\
& \quad+\left(\partial_{\omega_{1}}^{\prime}-2 x_{1}^{\prime} \partial_{t_{1}^{\prime}}\right)\left(\partial_{\omega_{2}}^{\prime}-2 x_{2}^{\prime} \partial_{t_{1}^{\prime}}\right) \\
&=: \frac{1}{2} \Delta_{K}+\hat{\mathcal{A}}_{2} \hat{\mathcal{A}}_{4},
\end{aligned}
$$

where $\Delta_{K}=(1 / 2)\left(\left(\hat{\mathcal{A}}_{2}\right)^{2}+\left(\hat{\mathcal{A}}_{3}\right)^{2}+\left(\hat{\mathcal{A}}_{3}\right)^{2}+\left(\hat{\mathcal{A}}_{4}\right)^{2}\right)$ equals the Kohn's Laplacian (Gaveau 1977), on the group $H_{5}$, which is a sum of the four horizontal left-invariant vector fields on $H_{5}$ (Gaveau 1977; Duits et al. 2009). Note that $\Delta_{K}$ is also the Kohn's Laplacian on the group $H_{3} \times H_{3}$ (with extra imposed identification $x_{1}^{\prime}=x_{2}^{\prime}$ ). If we neglect the crossterm $\hat{\mathcal{A}}_{2} \hat{\mathcal{A}}_{4}$ in the generator we get the following approximation 


$$
\begin{aligned}
\bar{p}_{t}^{D_{33}, D_{44}=D_{55} ; \mathbb{R}^{3} \rtimes S^{2}}(x, y, z, \tilde{\mathbf{n}}(\tilde{\beta}, \tilde{\gamma})) \\
\approx \bar{p}_{t}^{D_{33}, D_{44}=D_{55} ;(S E(2))_{0}}(z / 2, x, \tilde{\beta}) \\
\quad \cdot \bar{p}_{t}^{D_{33}, D_{44}=D_{55} ;(S E(2))_{0}}(z / 2,-y, \tilde{\gamma}) .
\end{aligned}
$$

So similar to the contour-completion kernel on $\mathbb{R}^{3} \rtimes S^{2}$ derived in the previous section, recall (65), the Heisenbergapproximation kernel on $\mathbb{R}^{3} \rtimes S^{2}$ is a direct product of two Heisenberg-approximation kernels on $(S E(2))_{0}$.

Now since the Heisenberg approximation kernel $\bar{p}_{t}^{D_{33}, D_{44} ;(S E(2))_{0}}$ is for reasonable parameter settings (that is $\left.0<\frac{D_{44}}{D_{33}} \ll 1\right)$ close to the exact kernel $\bar{p}_{t}^{D_{33}, D_{44} \text {; (SE (2)) }}$ we heuristically propose for these reasonable parameter settings the same direct-product approximation for the exact contour-enhancement kernels on $\mathbb{R}^{3} \rtimes S^{2}$ :

$$
\begin{aligned}
p_{t}^{D_{33}, D_{44}=D_{55} ; \mathbb{R}^{3} \rtimes S^{2}}(x, y, z, \tilde{\mathbf{n}}(\tilde{\beta}, \tilde{\gamma})) \\
\approx N\left(D_{33}, D_{44}, t\right) \cdot p_{t}^{D_{33}, D_{44} ;(S E(2))}(z / 2, x, \tilde{\beta}) \\
\cdot p_{t}^{D_{33}, D_{44} ;(S E(2))}(z / 2,-y, \tilde{\gamma}),
\end{aligned}
$$

where $N\left(D_{33}, D_{44}, t\right) \approx \frac{8}{\sqrt{2}} \sqrt{\pi} t \sqrt{t D_{33}} \sqrt{D_{33} D_{44}}$ takes care of $\mathbb{L}_{1}\left(\mathbb{R}^{3} \rtimes S^{2}\right)$-normalization. In Duits and Franken (2010), Citti and Sarti (2006) one can find the exact solutions of the Green's function $\bar{p}_{t}^{D_{33}, D_{44} ;(S E(2))_{0}}$ related to the Green's function (Gaveau 1977) on $H$ (3) by means of a coordinate transform, but these exact formulae are not as tangible as the following asymptotical formulas:

$$
\begin{gathered}
\bar{p}_{t}^{D_{33}, D_{44} ; S E(2)}(x, y, \theta) \\
\equiv \frac{1}{32 \pi t^{2} c^{4} D_{44} D_{33}} e^{-\frac{1}{4 t c^{2}} \sqrt{\left(\frac{x^{2}}{D_{33}}+\frac{\theta^{2}}{D_{44}}\right)^{2}+\frac{\left|y-\frac{x \theta}{2}\right|^{2}}{D_{44} D_{33}}}} \\
p_{t}^{D_{33}, D_{44} ; S E(2)}(x, y, \theta) \equiv \frac{1}{32 \pi t^{2} c^{4} D_{44} D_{33}} e^{-\frac{\sqrt{E N(x, y, \theta)}}{4 c^{2} t}}
\end{gathered}
$$

where we use short notation for the numerator of an exponent

$$
\begin{aligned}
E N(x, y, \theta)= & \left(\frac{\theta^{2}}{D_{44}}+\frac{\theta^{2}(y-(-x \sin \theta+y \cos \theta))^{2}}{4(1-\cos (\theta))^{2} D_{33}}\right)^{2} \\
& +\frac{1}{D_{44} D_{33}}\left|\frac{\theta((x \cos \theta+y \sin \theta)-x)}{2(1-\cos \theta)}\right|^{2}
\end{aligned}
$$

and which for $\theta=0$ equals

$E N(x, y, 0)=\left(\frac{x^{2}}{D_{33}}\right)^{2}+\frac{|y|^{2}}{D_{44} D_{33}}$.

The estimates (70) are globally sharp, with $\frac{1}{2} \leq c \leq \sqrt[4]{2}$, for details see Duits and Franken (2010, Chap. 5.4).
For the purpose of numerical computation, we simplify $E N(x, y, \theta)$ to

$$
\begin{aligned}
E N(x, y, \theta)= & \left(\frac{\theta^{2}}{D_{44}}+\frac{\left(\frac{\theta y}{2}+\frac{\theta / 2}{\tan (\theta / 2)} x\right)^{2}}{D_{33}}\right)^{2} \\
& +\frac{1}{D_{44} D_{33}}\left(\frac{-x \theta}{2}+\frac{\theta / 2}{\tan (\theta / 2)} y\right)^{2}
\end{aligned}
$$

where one can use the estimate $\frac{\theta / 2}{\tan (\theta / 2)} \approx \frac{\cos (\theta / 2)}{1-\left(\theta^{2} / 24\right)}$ for $|\theta|<\frac{\pi}{10}$ to avoid numerical errors.

\subsection{Gaussian Estimates for the Heat-kernels on $S E(3)$}

According to the general theory (ter Elst and Robinson 1998) the heat-kernels $p_{t}^{S E(3) ; q, \mathbf{D}=\operatorname{diag}\left\{0,0, D_{33}, D_{44}, D_{55}, 0\right\}}$ : $(\operatorname{SE}(3))_{q} \rightarrow \mathbb{R}^{+}$(i.e. kernels for contour enhancement whose convolutions yield horizontal ${ }^{9}$ diffusion on $\left.(S E(3))_{q}\right)$ on the parameterized class of groups $(S E(3))_{q}, q \in[0,1]$ in between $S E(3)$ and its nilpotent Heisenberg approximation $(S E(3))_{0}$ satisfy the following Gaussian estimates (for $\mathbf{D}$ isomorphic to the $3 \times 3$ identity matrix $I_{3}, \mathbf{D}=$ $\operatorname{diag}\{0,0,1,1,1,0\})$

$C_{1} e^{-\frac{C_{2}\|g\|_{q}^{2}}{4 t}} \leq p_{t}^{S E(3) ; q, \mathbf{D} \equiv I_{3}}(g) \leq C_{3} e^{-\frac{C_{4}\|g\|_{q}^{2}}{4 t}}$,

with $0<C_{1}<C_{3}$ and $0<C_{4}<C_{2}$, where the norm $\|\cdot\|_{q}$ : $(\operatorname{SE}(3))_{q} \rightarrow \mathbb{R}^{+}$is given by

$\|g\|_{q}=\left|\log _{(S E(3))_{q}}(g)\right|_{q}$,

where $\log _{(S E(3))_{q}}:(S E(3))_{q} \rightarrow T_{e}\left((S E(3))_{q}\right)$ is the logarithmic mapping on $(S E(3))_{q}$ (which we computed explicitly for $(S E(3))_{q=1}=S E(3)$ in Sect. 5.1 and which we will compute for $q=0$ as well) and where the weighted modulus, (ter Elst and Robinson 1998), in our special case of interest is given by

$$
\left|\sum_{i=1}^{6} c_{q}^{i} A_{i}^{q}\right|_{q}=\sqrt{\left|c_{q}^{1}\right|+\left|c_{q}^{2}\right|+\left|c_{q}^{6}\right|+\left|c_{q}^{3}\right|^{2}+\left|c_{q}^{4}\right|^{2}+\left|c_{q}^{5}\right|^{2}},
$$

where $c_{q}^{i} \in \mathbb{R}$ and where we recall our weighting given in (59). However, similar to our work (Duits and Franken 2010, Chap. 5.4) on estimating heat-kernels on $S E(2)$, we estimate the weighted modulus by an equivalent differentiable modulus:

$$
\begin{aligned}
& \left|\sum_{i=1}^{6} c_{q}^{i} A_{i}^{q}\right|^{q} \\
& \quad=\sqrt[4]{\left|c_{q}^{1}\right|^{2}+\left|c_{q}^{2}\right|^{2}+\left|c_{q}^{6}\right|^{2}+\left(\left|c_{q}^{3}\right|^{2}+\left|c_{q}^{4}\right|^{2}+\left|c_{q}^{5}\right|^{2}\right)^{2}},
\end{aligned}
$$

${ }^{9}$ Horizontal diffusion in $S E(3)$ is diffusion which takes place along horizontal curves in $\mathbb{R}^{3} \rtimes S^{2} \hookrightarrow S E(3)$. Recall Definition 5 . 
where we note that $\sqrt[4]{6}|g|^{q} \geq|g|_{q} \geq|g|^{q}$ for all $g \in$ $(\operatorname{SE}(3))_{q}, q \in[0,1]$.

Now suppose $\left(c_{q}^{1}, \ldots, c_{q}^{6}\right)=\left(c_{q}^{1}(g), \ldots, c_{q}^{6}(g)\right):=$ $\log _{(S E(3))_{q}}(g)$, then there exist constants $0<\tilde{C}_{1}<\tilde{C}_{3}$ and $0<\tilde{C}_{4}<\tilde{C}_{2}$ such that the following Gaussian estimates hold:

$$
\begin{aligned}
& \tilde{C}_{1} e^{-\frac{\tilde{C}_{2} \sqrt{\left|c_{q}^{1}\right|^{2}+\left|c_{q}^{2}\right|^{2}+\left|c_{q}^{6}\right|^{2}+\left(\left|c_{q}^{3}\right|^{2}+\left|c_{q}^{4}\right|^{2}+\left|c_{q}^{5}\right|^{2}\right)^{2}}}{4 t}} \\
& \quad \leq p_{t}^{S E(3) ; q, \mathbf{D} \equiv I_{3}}(g) \\
& \quad \leq \tilde{C}_{3} e^{-\frac{\tilde{C}_{4} \sqrt{\left|c_{q}^{1}\right|^{2}+\left|c_{q}^{2}\right|^{2}+\left|c_{q}^{6}\right|^{2}+\left(\left|c_{q}^{3}\right|^{2}+\left|c_{q}^{4}\right|^{2}+\left|c_{q}^{5}\right|^{2}\right)^{2}}}{4 t}},
\end{aligned}
$$

where we again use short notation $c_{q}^{i}=c_{q}^{i}(g), i=1, \ldots, 6$. Now, from the applied point of view $\mathbf{D}=\operatorname{diag}\{0,0,1,1,1,0\}$ is an un-realistic situation and only for $q=0$ there exist dilations on the group $(S E(3))_{q}$ so that we can easily generalize the estimates to the diagonal case $\mathbf{D}=$ $\operatorname{diag}\left\{0,0, D_{33}, D_{44}, D_{55}, 0\right\}$.

Since $(S E(3))_{0}$ is a nilpotent Lie-group isomorphic to the matrix group given by (60) (where we take the limit $q \downarrow 0$ ) it is not difficult (this is much easier than the case $q=1$, recall Sect. 5.1) to compute the exponent (recall (61)):

$$
\begin{aligned}
\exp & \left(\begin{array}{cccc}
0 & -c_{0}^{6} & c_{0}^{5} & c_{0}^{1} \\
0 & 0 & -c_{0}^{4} & c_{0}^{2} \\
0 & 0 & 0 & c_{0}^{3} \\
0 & 0 & 0 & 0
\end{array}\right) \\
& =\left(\begin{array}{cccc}
1 & -c_{0}^{6} & c_{0}^{5}+\frac{1}{2} c_{0}^{4} c_{0}^{6} & c_{0}^{1}+\frac{1}{2}\left(c_{0}^{3} c_{0}^{5}-c_{0}^{2} c_{0}^{6}\right)+\frac{1}{6} c_{0}^{3} c_{0}^{4} c_{0}^{6} \\
0 & 1 & -c_{0}^{4} & c_{0}^{2}-\frac{1}{2} c_{0}^{3} c_{0}^{4} \\
0 & 0 & 1 & c_{0}^{3} \\
0 & 0 & 0 & 1
\end{array}\right) \\
& =\left(\begin{array}{cccc}
1 & -\tilde{\alpha} & \tilde{\beta} & x \\
0 & 1 & -\tilde{\gamma} & y \\
0 & 0 & 1 & z \\
0 & 0 & 0 & 1
\end{array}\right)
\end{aligned}
$$

and inverting these relations we find the simple formulas for the functions $c_{0}^{i}$ that we use in our estimates (73)

$c_{0}^{1}(g)=x+\frac{1}{2} y \tilde{\alpha}-\frac{1}{2} z \tilde{\beta}+\frac{1}{3} z \tilde{\alpha} \tilde{\gamma}, \quad c_{0}^{4}(g)=\tilde{\gamma}$,

$c_{0}^{2}(g)=y+\frac{1}{2} z \tilde{\gamma}, \quad c_{0}^{5}(g)=\tilde{\beta}-\frac{1}{2} \tilde{\alpha} \tilde{\gamma}$,

$c_{0}^{3}(g)=z, \quad c_{0}^{6}(g)=\tilde{\alpha}$

defined for all $g=\left(x, y, z,\left(\begin{array}{ccc}1 & \tilde{\alpha} & -\tilde{\beta} \\ 0 & 1 & \tilde{\gamma} \\ 0 & 0 & 1\end{array}\right)\right) \in(S E(3))_{0}$. By our embedding $\mathbb{R}^{3} \rtimes S^{2}$ into $S E(3)$, we must set $c_{0}^{6}=\tilde{\alpha}=0$.
Consequently, for the Heisenberg approximations of the diffusion kernels we have

$$
\begin{aligned}
& \tilde{C}_{1} e^{-\frac{\tilde{C}_{2} \sqrt{\left|x-\frac{1}{2} z \tilde{\beta}\right|^{2}+\left|y+\frac{1}{2} z \tilde{\gamma}\right|^{2}+\left(|z|^{2}+|\tilde{\gamma}|^{2}+|\tilde{\beta}|^{2}\right)^{2}}}{4 t}} \\
& \quad \leq \bar{p}_{t}(x, y, z, \tilde{n}(\tilde{\beta}, \tilde{\gamma})) \\
& \quad \leq \tilde{C}_{3} e^{-\frac{\tilde{C}_{4} \sqrt{\left|x-\frac{1}{2} z \tilde{\beta}\right|^{2}+\left|y+\frac{1}{2} z \tilde{\gamma}\right|^{2}+\left(|z|^{2}+|\tilde{\gamma}|^{2}+|\tilde{\beta}|^{2}\right)^{2}}}{4 t}},
\end{aligned}
$$

where we used short notation $\bar{p}_{t}=\bar{p}_{t}^{(S E(3))_{0}, \mathbf{D} \equiv I_{3}}$ : $\mathbb{R}^{3} \times S^{2} \rightarrow \mathbb{R}^{+}$for the Heisenberg approximation $q=0$ of the contour enhancement kernel with $D_{33}=D_{44}=D_{55}=$ 1. Now by application of the following dilation:

$z^{\prime}=\frac{z}{\sqrt{D_{33}}}, \quad x^{\prime}=\frac{x}{\sqrt{D_{33} D_{44}}}$,

$\tilde{\beta}^{\prime}=\frac{\tilde{\beta}}{\sqrt{D_{44}}}, \quad y^{\prime}=\frac{y}{\sqrt{D_{33} D_{44}}}, \quad \tilde{\gamma}^{\prime}=\frac{\tilde{\gamma}}{\sqrt{D_{44}}}$

the generator of the corresponding diffusion on $\mathbb{R}^{3} \rtimes S^{2}$ for the general case where $\mathbf{D}=\operatorname{diag}\left\{0,0, D_{33}, D_{44}, D_{55}=\right.$ $\left.D_{44}, 0\right\}$ relates to the diffusion generator for the case $\mathbf{D}=$ $\operatorname{diag}\{0,0,1,1,1,0\}$, recall (62):

$$
\begin{gathered}
\sum_{i=3}^{5} D_{i i}\left(\mathcal{A}_{i}^{0}\right)^{2}=D_{33}\left(\tilde{\beta} \partial_{x}-\tilde{\gamma} \partial_{y}+\partial_{z}\right)^{2} \\
+D_{44}\left(\partial_{\tilde{\beta}}\right)^{2}+D_{44}\left(\partial_{\tilde{\beta}}\right)^{2} \\
\leftrightarrow\left(\tilde{\beta}^{\prime} \partial_{x}^{\prime}-\tilde{\gamma}^{\prime} \partial_{y}^{\prime}+\partial_{z}^{\prime}\right)^{2}+\left(\partial_{\tilde{\beta}^{\prime}}\right)^{2}+\left(\partial_{\tilde{\beta}^{\prime}}\right)^{2},
\end{gathered}
$$

consequently, we find the following estimates for the general Heisenberg approximation kernels

$$
\begin{aligned}
& \tilde{C}_{1} e^{-\frac{\tilde{C}_{3} \sqrt{\frac{\left|c 0^{1}\right|^{2}+\left|c_{0}^{2}\right|^{2}}{D_{33} D_{44}}+\left(\frac{\left(c_{0}^{3}\right)^{2}}{D_{33}}+\frac{\left|c_{0}^{4}\right|^{2}+\left|c_{0}^{5}\right|^{2}}{D_{44}}\right)^{2}}}{4 t}} \\
& \leq \bar{p}_{t}^{\mathbf{D}, q=0}(\mathbf{x}, \tilde{n}(\tilde{\beta}, \tilde{\gamma})) \\
& \leq \tilde{C}_{2} e^{-\frac{\tilde{c}_{4} \sqrt{\frac{\left|c 0^{1}\right|^{2}+\left|c_{0}^{2}\right|^{2}}{D_{33} D_{44}}+\left(\frac{\left(c_{0}^{3}\right)^{2}}{D_{33}}+\frac{\left|c_{0}^{4}\right|^{2}+\left|c_{0}^{5}\right|^{2}}{D_{44}}\right)^{2}}}{4 t}},
\end{aligned}
$$

where we used short notation $c_{0}^{k}=c_{0}^{k}\left(\mathbf{x}, R_{\tilde{\gamma}, \tilde{\beta}, 0}\right), k=$ $1, \ldots, 5, \mathbf{x}=(x, y, z)^{T} \in \mathbb{R}^{3}$, recall (72).

In fact in Duits and Franken (2009, Chap. 6.2) it is shown that the constants $\tilde{C}_{3}, \tilde{C}_{4}$ are very close and that a reasonably sharp approximation and upperbound of the horizontal diffusion kernel on $\mathbb{R}^{3} \rtimes S^{2}$ is given by

$$
\begin{aligned}
p_{t}^{\mathbf{D}} & =\operatorname{diag}\left\{0,0, D_{33}, D_{44}, D_{55}, 0\right\} \\
& \approx \frac{1}{(4 \pi, \tilde{n}(\tilde{\beta}, \tilde{\gamma}))} \\
& \frac{1}{\left.t^{2} D_{33} D_{44}\right)^{2}} e^{-\frac{\sqrt{\left.\frac{|c|}{1}\right|^{2}+\left|c^{2}\right|^{2}} D_{33} D_{44}+\frac{\left|c^{6}\right|^{2}}{D_{44}}+\left(\frac{\left(c^{3}\right)^{2}}{D_{33}}+\frac{\left|c^{4}\right|^{2}+\left|c^{5}\right|^{2}}{D_{44}}\right)^{2}}{4 t}},
\end{aligned}
$$

where we again use short notation $c^{k}:=c_{q=1}^{k}\left(\mathbf{x}, R_{\tilde{\gamma}, \tilde{\beta}, 0}\right)$, $k=1, \ldots, 6$. Recall from Sect. 5.1 that these constants 
are computed by the logarithm, (57), on $S E(3)$ or more explicitly by (55) and (56). The latter two equalities are analogously expressed in the second coordinate chart yielding formulas for the functions $c^{k}$ in (75):

$$
\begin{aligned}
& \tilde{q}=\arcsin \sqrt{\cos ^{4}(\tilde{\gamma} / 2) \sin ^{2}(\tilde{\beta})+\cos ^{2}(\tilde{\beta} / 2) \sin ^{2}(\tilde{\gamma}),} \\
& \mathbf{c}^{(2)=}\left(c^{4}, c^{5}, c^{6}\right)^{T} \\
&=\frac{\tilde{q}}{\sin (\tilde{q})}\left(\sin \tilde{\gamma} \cos ^{2}\left(\frac{\tilde{\beta}}{2}\right), \sin \tilde{\beta} \cos ^{2}\left(\frac{\tilde{\gamma}}{2}\right),\right. \\
&\left.\frac{1}{2} \sin \tilde{\gamma} \sin \tilde{\beta}\right)^{T}, \\
& \mathbf{c}^{(1)}=\left(c^{1}, c^{2}, c^{3}\right)^{T} \\
&= \mathbf{x}-\frac{1}{2} \mathbf{c}^{(2)} \times \mathbf{x} \\
&+\tilde{q}^{-2}\left(1-\left(\frac{\tilde{q}}{2}\right) \cot \left(\frac{\tilde{q}}{2}\right)\right) \mathbf{c}^{(2)}\left(\mathbf{c}^{(2)} \times \mathbf{x}\right) .
\end{aligned}
$$

These functions $c^{k}$ (the case $q=1$ ) are indeed consistent with the functions $c_{0}^{k}$ (the case $q=0$ ) in the sense that $\lim _{q \downarrow 0} q^{-w_{k}} c^{k}\left(x q^{w_{1}}, y q^{w_{2}}, z q^{w_{3}}, \tilde{\mathbf{n}}\left(q^{w_{4}} \tilde{\beta}, q^{w_{5}} \tilde{\beta}\right)\right)=$ $c_{0}^{k}(x, y, z, \tilde{\beta}, \tilde{\gamma})$, where we recall (59) for $k=1,2, \ldots, 5$.

\section{Implementation of the Left-Invariant Derivatives and $\mathbb{R}^{3} \times S^{2}$-Diffusion}

In our implementations we do not use the two charts (among which the Euler-angles parametrization) of $S^{2}$ because this would involve cumbersome and expensive bookkeeping of mapping the coordinates from one chart to the other (which becomes necessary each time the singularities (3) and (6) are reached). Instead we recall that the left-invariant vector fields on HARDI-orientation scores $\tilde{U}: S E(3) \rightarrow \mathbb{R}$, which by definition (recall Definition 4 ) automatically satisfy

$\tilde{U}\left(\mathbf{y}, R R_{\mathbf{e}_{z, \alpha}}\right)=\tilde{U}(\mathbf{y}, R), \quad$ for all $\mathbf{y} \in \mathbb{R}^{3}$,

are constructed by the derivative of the right-regular representation

$$
\begin{aligned}
\mathcal{A}_{i} \tilde{U}(g) & =\left(\mathrm{d} \mathcal{R}\left(A_{i}\right) \tilde{U}\right)(g) \\
& =\lim _{t \downarrow 0} \frac{\tilde{U}\left(g e^{t A_{i}}\right)-\tilde{U}(g)}{t} \\
& =\lim _{t \downarrow 0} \frac{\tilde{U}\left(g e^{t A_{i}}\right)-\tilde{U}\left(g e^{-t A_{i}}\right)}{2 t},
\end{aligned}
$$

where in the numerics we can take finite step-sizes in the right-hand side. Now in order to avoid a redundant computation we can also avoid taking the de-tour via HARDIorientation scores and actually work with the left-invariant vector fields on the HARDI data itself. To this end we need the consistent right-action $\mathfrak{R}$ of $S E(3)$ acting on the space of HARDI images $\mathbb{L}_{2}\left(\mathbb{R}^{3} \rtimes S^{2}\right)$. Let $H$ denote the space of HARDI-orientation scores, i.e. $H$ is the space of quadratic integrable functions on the group $S E(3)$ which satisfy (77). To construct this consistent right-action on $H$ we first define $S: \mathbb{L}_{2}\left(\mathbb{R}^{3} \rtimes S^{2}\right) \rightarrow H$, by

$(S U)(\mathbf{x}, R)=\tilde{U}(\mathbf{x}, R)=U\left(\mathbf{x}, R \mathbf{e}_{z}\right)$.

This mapping is injective and its left-inverse is given by $\left(S^{-1} \tilde{U}\right)(\mathbf{x}, \mathbf{n})=\tilde{U}\left(\mathbf{x}, R_{\mathbf{n}}\right)$, where again $R_{\mathbf{n}} \in S O(3)$ is some rotation such that $R_{\mathbf{n}} \mathbf{e}_{z}=\mathbf{n}$. Now the consistent right-action $\mathfrak{R}: S E(3) \rightarrow B\left(\mathbb{L}_{2}\left(\mathbb{R}^{3} \rtimes S^{2}\right)\right.$ ), where $B\left(\mathbb{L}_{2}\left(\mathbb{R}^{3} \rtimes S^{2}\right)\right)$ stands for all bounded linear operators on the space of HARDI images, is given by

$$
\begin{aligned}
\left(\Re_{(\mathbf{x}, R)} U\right)(\mathbf{y}, \mathbf{n}) & =\left(S^{-1} \circ \mathcal{R}_{(\mathbf{x}, R)} \circ S U\right)(\mathbf{y}, \mathbf{n}) \\
& =U\left(R_{\mathbf{n}} \mathbf{x}+\mathbf{y}, R_{\mathbf{n}} R \mathbf{e}_{z}\right) .
\end{aligned}
$$

This yields the left-invariant vector fields (directly) on sufficiently smooth HARDI images:

$$
\begin{aligned}
\mathcal{A}_{i} U(\mathbf{y}, \mathbf{n}) & =\left(\mathrm{d} \Re\left(A_{i}\right) U\right)(\mathbf{y}, \mathbf{n}) \\
& =\lim _{h \downarrow 0} \frac{\left(\Re_{e^{h A_{i}}} U\right)(\mathbf{y}, \mathbf{n})-U(\mathbf{y}, \mathbf{n})}{h} \\
& =\lim _{h \downarrow 0} \frac{\left(\Re_{e^{h A_{i}}} U\right)(\mathbf{y}, \mathbf{n})-\left(\Re_{e^{-h A_{i}}} U\right)(\mathbf{y}, \mathbf{n})}{2 h} .
\end{aligned}
$$

Now in our algorithms we take finite step-sizes and elementary computations (using the exponent given by (54)) yield the following simple expressions for the (horizontal) leftinvariant vector fields:

$$
\begin{aligned}
& \mathcal{A}_{1} U(\mathbf{y}, \mathbf{n}) \approx \frac{U\left(\mathbf{y}+h R_{\mathbf{n}} \mathbf{e}_{x}, \mathbf{n}\right)-U\left(\mathbf{y}-h R_{\mathbf{n}} \mathbf{e}_{x}, \mathbf{n}\right)}{2 h}, \\
& \mathcal{A}_{2} U(\mathbf{y}, \mathbf{n}) \approx \frac{U\left(\mathbf{y}+h R_{\mathbf{n}} \mathbf{e}_{y}, \mathbf{n}\right)-U\left(\mathbf{y}-h R_{\mathbf{n}} \mathbf{e}_{y}, \mathbf{n}\right)}{2 h}, \\
& \mathcal{A}_{3} U(\mathbf{y}, \mathbf{n}) \approx \frac{U\left(\mathbf{y}+h R_{\mathbf{n}} \mathbf{e}_{z}, \mathbf{n}\right)-U\left(\mathbf{y}-h R_{\mathbf{n}} \mathbf{e}_{z}, \mathbf{n}\right)}{2 h}, \\
& \mathcal{A}_{4} U(\mathbf{y}, \mathbf{n}) \approx \frac{U\left(\mathbf{y}, R_{\mathbf{n}} R_{\mathbf{e}_{x}, h} \mathbf{e}_{z}\right)-U\left(\mathbf{y}, R_{\mathbf{n}} R_{\mathbf{e}_{x},-h} \mathbf{e}_{z}\right)}{2 h}, \\
& \mathcal{A}_{5} U(\mathbf{y}, \mathbf{n}) \approx \frac{U\left(\mathbf{y}, R_{\mathbf{n}} R_{\mathbf{e}_{y}, h} \mathbf{e}_{z}\right)-U\left(\mathbf{y}, R_{\mathbf{n}} R_{\mathbf{e}_{y},-h} \mathbf{e}_{z}\right)}{2 h} .
\end{aligned}
$$

The left-invariant vector fields $\left\{\mathcal{A}_{1}, \mathcal{A}_{2}, \mathcal{A}_{4}, \mathcal{A}_{5}\right\}$ clearly depend on the choice of $R_{\mathbf{n}} \in S O(3)$ which maps $R_{\mathbf{n}} \mathbf{e}_{z}=$ n. Now functions in the space $H$ are $\alpha$-right invariant, so thereby we may assume that $R$ can be written as $R=R_{\mathbf{e}_{x}, \tilde{\gamma}} R_{\mathbf{e}_{y}, \tilde{\beta}}$, now if we choose $R_{\mathbf{n}}$ again such that $R_{\mathbf{n}}=R_{\tilde{\mathbf{n}}(\tilde{\beta}, \tilde{\gamma})}=R_{\mathbf{e}_{x}, \tilde{\gamma}} R_{\mathbf{e}_{y}, \tilde{\beta}} R_{\mathbf{e}_{z}, \tilde{\alpha}=\alpha_{0}=0}$ then we take consistent sections in $S O(3) / S O(2)$ and we get full invertibility $S^{-1} \circ S=S \circ S^{-1}=\mathcal{I}$. 
In our diffusion schemes, however, the choice of representant $R_{\mathbf{n}}$ is irrelevant, because we impose $\alpha$-right invariance (31) on the diffusion generator (which in the linear case boils down to (34)) and as a result we have $D_{44}=D_{55}, D_{11}=D_{22}$. The thereby obtained operators $\left(\mathcal{A}_{4}\right)^{2}+\left(\mathcal{A}_{5}\right)^{2}=\left.\Delta_{S^{2}}\right|_{H}$ and $\mathcal{A}_{1}^{2}+\mathcal{A}_{2}^{2}=\Delta_{\mathbb{R}^{3}}-\mathcal{A}_{3}^{2}$ are invariant under transformations of the type $\underline{\mathcal{A}} \mapsto Z_{\alpha_{0}} \underline{\mathcal{A}}$ for all $\alpha_{0} \in[0,2 \pi)$, recall $(32)$.

In the computation of (78) one would have liked to work with discrete subgroups of $S O(3)$ acting on $S^{2}$ in order to avoid interpolations, but unfortunately the platonic solid with the largest amount of vertices (only 20) is the dodecahedron and the platonic solid with the largest amount of faces (again only 20) is the icosahedron. Nevertheless, we would like to sample the 2-sphere such that the distance between sampling points should be as equal as possible and simultaneously the area around each sample point should be as equal as possible. Therefore we follow the common approach by regular triangulations (i.e. each triangle is regularly divided into $(o+1)^{2}$ triangles $)$ of the icosahedron, followed by a projection on the sphere. This leads to $N_{o}=2+10(o+1)^{2}$ vertices. We typically considered $o=1,2,3$, for further details on uniform spherical sampling, see Franken (2008, Chap. 7.8.1).

For the required interpolations to compute (78) within our spherical sampling there are two simple options. Either one uses a triangular interpolation of using the three closest sampling points, or one uses a discrete spherical harmonic interpolation. The disadvantage of the first and simplest approach is that it introduces additional blurring, whereas the second approach can lead to overshoots and undershoots. In the latter approach a $\pi$-symmetric function on the sphere only requires even values for $l \in\{0,2,4, \ldots, L\}$ in which case the total amount of spherical harmonics is $n_{S H}=\frac{1}{2}(L+1)(L+2)$. Although, there exist more efficient and accurate algorithms for discrete harmonic transforms (DSHT), (Driscoll and Healy 1994; Kunis and Potts 2003), we next give a brief explanation of the basic algorithm we used. To this end we first recall that the continuous spherical Harmonic transform is given by

$$
\begin{aligned}
(\operatorname{SHT}(f))(l, m) & =\left(Y_{m}^{l}, f\right)_{\mathbb{L}_{2}\left(S_{2}\right)} \\
& =\int_{0}^{2 \pi} \int_{0}^{\pi} \overline{Y_{m}^{l}(\beta, \gamma)} f(\mathbf{n}(\beta, \gamma)) \sin \beta \mathrm{d} \beta \mathrm{d} \gamma .
\end{aligned}
$$

The spherical harmonics (38) form a complete orthonormal basis in $\mathbb{L}_{2}\left(S^{2}\right)$, so the inverse is given by

$f(\mathbf{n}(\beta, \gamma))=\sum_{l=0}^{\infty} \sum_{m=-l}^{l}(\operatorname{SHT}(f))(l, m) Y_{m}^{l}(\beta, \gamma)$

for almost every $\beta \in[0, \pi)$ and almost every $\gamma \in[0,2 \pi)$. As mentioned before (in Sect. 4.1) the function $f$ becomes a regular smooth function (which is defined everywhere) if we apply a slight diffusion on the 2 -sphere:

$$
\begin{aligned}
& e^{t_{\text {reg }} \Delta_{S^{2}}} f(\mathbf{n}(\beta, \gamma)) \\
& \quad=\sum_{l=0}^{\infty} \sum_{m=-l}^{l} e^{-t_{\text {reg }}(l+1) l}(\operatorname{SHT}(f))(l, m) Y_{m}^{l}(\beta, \gamma),
\end{aligned}
$$

with $0<t_{\text {reg }} \ll 1$. Next we explain two basic discrete versions of the SHT-transform. Both can be used in a finite difference scheme requiring discretization of for example $\Delta_{S^{2}}=\left(\mathcal{A}_{4}\right)^{2}+\left.\left(\mathcal{A}_{5}\right)^{2}\right|_{H}$.

\subsection{DSHT and DISHT}

There exist two basic approaches to discretize the continuous spherical harmonic transform. Either one considers (79) as a starting point and approximates the integral by a Riemann sum taking care of the surface measure, yielding the DSHT-transform and its pseudo-inverse. Or one considers (80) as a starting point yielding the DISHT-transform and its pseudoinverse. The first approach is exact on the grid if the number of spherical harmonics is larger than the number of samples $n_{S H} \geq N_{o}$, whereas the second approach is exact on the grid if $n_{S H} \leq N_{o}$.

The pseudoinverse $\mathbf{Q}^{+}$of a matrix $\mathbf{Q} \in \mathbb{R}^{m \times n}$ is defined by $\mathbf{Q}^{+} \mathbf{x}=\lim _{\delta \downarrow 0}\left(\mathbf{Q}^{\dagger} \mathbf{Q}+\delta I\right)^{-1} \mathbf{Q}^{\dagger} \mathbf{x}$, with $\mathbf{Q}^{\dagger}=\overline{\mathbf{Q}^{T}}$. Iff the columns of $\mathbf{Q}$ are linearly independent, then $\mathbf{Q}^{+}=$ $\left(\mathbf{Q}^{\dagger} \mathbf{Q}\right)^{-1} \mathbf{Q}^{\dagger}$ and $\mathbf{Q}^{+} \mathbf{Q}=\mathbf{I}$.

Consider a "uniform" spherical sampling $\left\{\mathbf{n}_{k}\right\}_{k=1}^{N_{o}} \subset S^{2}$ such that the associated a $n_{S H} \times N_{o}$-matrix

$$
\begin{aligned}
& \mathbf{M}=\left[M_{k}^{j}\right]=\left[\frac{1}{\sqrt{C}} Y_{m(j)}^{l(j)}\left(\mathbf{n}_{k}\right)\right], \\
& \text { with } l(j)=\lfloor\sqrt{j-1}\rfloor \text { and } m(j)=j-(l(j))^{2}-l(j)-1
\end{aligned}
$$

and $C=\sum_{j=1}^{n_{S H}}\left|Y_{m(j)}^{l(j)}(0,0)\right|^{2}$ (chosen to ensure that $\mathbf{M}^{\dagger} \mathbf{M}$ has 1 on the diagonal), has linearly independent columns (so $N_{o} \leq n_{S H}$ ). Then the DSHT and its pseudo-inverse are given by

\section{$\operatorname{DSHT}[\mathbf{f}]=\overline{\mathbf{M}} \mathbf{\Lambda} \mathbf{f}$}

$\mathrm{DSHT}^{+}[\mathbf{s}]=\left((\overline{\mathbf{M}} \mathbf{\Lambda})^{\dagger} \overline{\mathbf{M}} \mathbf{\Lambda}\right)^{-1}(\overline{\mathbf{M}} \mathbf{\Lambda})^{\dagger} \mathbf{s}, \quad n_{S H} \geq N_{o}$,

where the matrix $\boldsymbol{\Lambda}=\operatorname{diag}\left\{\delta_{S^{2}}\left(\mathbf{n}_{1}\right), \ldots, \delta_{S^{2}}\left(\mathbf{n}_{N_{o}}\right)\right\}$ contains discrete surface measures $\delta_{S^{2}}\left(\mathbf{n}_{k}\right)$ given by

$\delta_{S^{2}}\left(\mathbf{n}_{k}\right)=\frac{1}{6} \sum_{i \neq k, j \neq k, i \neq j, i \sim j \sim k} A\left(\mathbf{n}_{i}, \mathbf{n}_{j}, \mathbf{n}_{k}\right)$,

where $i \sim j$ means that $\mathbf{n}_{i}$ and $\mathbf{n}_{j}$ are part of a locally smallest triangle in say the second order tessellation of an icosa- 
hedron and where the surface measure of the spherical projection of such a triangle is given by

$$
\begin{aligned}
& A\left(\mathbf{n}_{i}, \mathbf{n}_{j}, \mathbf{n}_{k}\right) \\
& =4 \arctan \left\{\tan \left(s_{i j k} / 2\right) \tan \left(\left(s_{i j k}-s_{i j}\right) / 2\right)\right. \\
& \left.\quad \cdot \tan \left(\left(s_{i j k}-s_{i k}\right) / 2\right) \tan \left(\left(s_{i j k}-s_{j k}\right) / 2\right)\right\}^{1 / 2},
\end{aligned}
$$

with $s_{i j k}=\frac{1}{2}\left(s_{i j}+s_{i k}+s_{j k}\right)$ and $s_{i j}=\arccos \left(\mathbf{n}_{i} \cdot \mathbf{n}_{j}\right)$. The DISHT (which follows by sampling of (80)) and its pseudoinverse DISHT $^{+}$are given by

$$
\begin{aligned}
& \operatorname{DISHT}[\mathbf{s}]=\mathbf{M}^{T} \mathbf{s} \\
& (\operatorname{DISHT})^{+}[\mathbf{f}]=\left(\overline{\mathbf{M}} \mathbf{M}^{T}\right)^{-1}(\overline{\mathbf{M}}) \mathbf{f}, \quad n_{S H} \leq N_{o} .
\end{aligned}
$$

The pseudo-inverse DISHT ${ }^{+}$is commonly used in HARDIDTI imaging (on separate glyphs) as initiated by Descoteaux et al. (2007) where the authors include a Tikhonov-type of regularization $\left(\overline{\mathbf{M}} \mathbf{M}^{T}+\gamma^{2} \operatorname{diag}\{l(j)(l(j)+1)\}\right)^{-1}(\overline{\mathbf{M}}) \mathbf{f}$, $\gamma>0$, within the transform DISHT ${ }^{+}$. This destroys (wellposed) invertibility on the grid but clearly it stabilizes the acquisition of low order spherical harmonic coefficients from in practice often incomplete spherical samplings. However, in our framework we would like to return from the spherical harmonic coefficients to the spherical sampling on say a 2 nd order tessellation of an icosahedron. Moreover, we would like to include the weighting factors $\delta_{S^{2}}\left(\mathbf{n}_{k}\right)$ (which satisfy $\left.\sum_{k=1}^{N_{o}} \delta_{S^{2}}\left(\mathbf{n}_{k}\right)=4 \pi\right)$ that compensate for differences in the surrounding areas of the sampling points. So we have two options for computing the left-invariant Laplacian on $S^{2}$ :

$$
\begin{aligned}
& \Delta_{S^{2}} W\left(\mathbf{y}, \mathbf{n}_{k}, t\right) \\
&=\sum_{l=0}^{\infty} \sum_{m=-l}^{l} l(l+1)(\operatorname{SHT}(f))(l, m) Y_{l, m}\left(\mathbf{n}_{k}\right) \\
& \approx {\left[\operatorname { D S H T } ^ { + } \left[j \mapsto l(j)(l(j)+1) e^{-t_{\text {reg }}(l(j)+1) l(j)}\right.\right.} \\
&\left.\left.\cdot \operatorname{DSHT}\left[k^{\prime} \mapsto W\left(\mathbf{y}, \mathbf{n}_{k}^{\prime}, t\right)\right](j)\right]\right][k] \\
& \approx \frac{1}{\delta_{S^{2}}\left(\mathbf{n}_{k}\right)}\left[\operatorname { D I S H T } \left[j \mapsto l(j)(l(j)+1) e^{-t_{\text {reg }}(l(j)+1) l(j)}\right.\right. \\
&\left.\left.\cdot \operatorname{DSHT}\left[k^{\prime} \mapsto W\left(\mathbf{y}, \mathbf{n}_{k}^{\prime}, t\right)\right](j)\right]\right][k],
\end{aligned}
$$

with regularizing parameter $0<t_{\text {reg }} \ll 1$. In order to stay close to the continuous setting we have applied the second option in our discrete experiments, although the second option would act entirely in the discrete setting (where $\mathrm{DSHT}^{+} \circ \mathrm{DSHT}=\mathbf{I}$ if $n_{S H} \geq N_{o}$ ). The two methods converge to each other if $n_{S H} \rightarrow \infty$, since

$$
\begin{aligned}
& \left(\mathbf{M}^{\dagger} \mathbf{M} \rightarrow \mathbf{I} \text { as } n_{S H} \rightarrow \infty\right) \\
& \quad \Rightarrow \quad\left(\mathrm{DSHT}^{+} \rightarrow \boldsymbol{\Lambda}^{-1} \mathbf{M}^{T}=\boldsymbol{\Lambda}^{-1} \text { DISHT as } n_{S H} \rightarrow \infty\right) .
\end{aligned}
$$
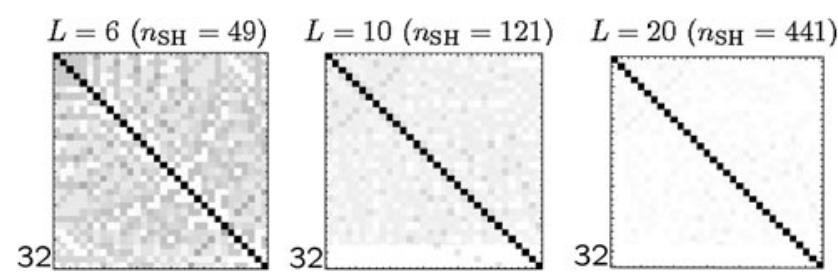

Fig. 6 Effect of increasing spherical harmonic bandwidth $L$ on the net operator matrix $\mathbf{M}^{\dagger} \mathbf{M}=\overline{\mathbf{M}}^{T} \mathbf{M}$ for the case $N_{o}=32$ (1st order tessellation of dodecahedron). If one takes higher order spherical harmonics than strictly required, reconstruction of the sampled function on the sphere improves. Note that $\mathbf{M}^{\dagger} \mathbf{M} \rightarrow \mathbf{I}$ as $n_{S H} \rightarrow \infty$

See Fig. 6. In practice one must be careful since if $n_{S H}$ becomes too large aliasing artifacts arise and a potential decrease of numerical instability arises. Therefore we included a regularization parameter $t_{\text {reg }}$ to guarantee stability. Typically $t_{\text {reg }}>0$ should be chosen very small, but not too small as the function $j \mapsto l(j)(l(j)+1) e^{-t_{\text {reg }}(l(j)+1)(l(j))} \times$ $\operatorname{DSHT}\left[k^{\prime} \mapsto W\left(\mathbf{y}, \mathbf{n}_{k}^{\prime}, t\right)\right](j)$ should nearly vanish at $j \geq N_{o}$ to avoid aliasing.

\subsection{Finite Difference Scheme for Linear $\mathbb{R}^{3} \rtimes S^{2}$ Diffusion}

The linear diffusion system on $\mathbb{R}^{3} \rtimes S^{2}$ can be rewritten as

$$
\begin{aligned}
\partial_{t} W(\mathbf{y}, \mathbf{n}, t)= & \left(D_{11}\left(\left(\mathcal{A}_{1}\right)^{2}+\left(\mathcal{A}_{2}\right)^{2}\right)\right. \\
& \left.+D_{33}\left(\mathcal{A}_{3}\right)^{2}+D_{44} \Delta_{S^{2}}\right) W(\mathbf{y}, \mathbf{n}, t)
\end{aligned}
$$

$W(\mathbf{y}, \mathbf{n}, 0)=U(\mathbf{y}, \mathbf{n})$.

This system is the Fokker-Planck equation of horizontal Brownian motion on $\mathbb{R}^{3} \rtimes S^{2}$ if $D_{11}=0$. Spatially, we take second order centered finite differences for $\left(\mathcal{A}_{1}\right)^{2},\left(\mathcal{A}_{2}\right)^{2}$ and $\left(\mathcal{A}_{3}\right)^{2}$, i.e. we applied the discrete operators in the righthand side of (78) twice (where we replaced $2 h \mapsto h$ to ensure direct-neighbors interaction), e.g. we have

$$
\begin{aligned}
& \left(\left(\mathcal{A}_{3}\right)^{2} W\right)(\mathbf{y}, \mathbf{n}, t) \\
& \approx \frac{W\left(\mathbf{y}+h R_{\mathbf{n}} \mathbf{e}_{z}, \mathbf{n}, t\right)-2 W(\mathbf{y}, \mathbf{n}, t)+W\left(\mathbf{y}-h R_{\mathbf{n}} \mathbf{e}_{z}, \mathbf{n}, t\right)}{h^{2}},
\end{aligned}
$$

where one can either apply the earlier mentioned interpolation methods (2nd order B-spline or (81)) or (as we did in our experiments) one first computes all second order finite differences on the cubic spatial grid and rotates them back to the spatial part of the moving frame of reference, (24), attached to $(\mathbf{y}, \mathbf{n})$. The spherical Laplacian $\Delta_{L B}$ is computed by means of (85) (second approximation). For efficiency, the chain of operators, DSHT-diag $\left\{l(l+1) e^{-t_{\text {reg }} l(l+1)}\right\}$-DISHT, is stored in a single $N_{o} \times N_{o}$-matrix, so that calculation of $\Delta_{S^{2}}$ consists of a simple matrix-vector multiplication. In our algorithm we apply a first order approximation in time 
$\partial_{t} W(\mathbf{y}, \mathbf{n}, t) \approx \frac{W(\mathbf{y}, \mathbf{n}, t+\Delta t)-W(\mathbf{y}, \mathbf{n}, t)}{\Delta t}$,

where we choose $\Delta t$ small enough such that the algorithm is stable. As we have shown in Duits and Franken (2009, Chap. 7.2, App. B) sharp upper bounds on $\Delta t$ which guarantee stability are given by

$$
\begin{aligned}
& \Delta t \leq \frac{h^{2}}{4 D_{11}+2 D_{33}+D_{44} h^{2} \frac{L(L+1)}{2 e^{t_{\text {reg }}(L+1)}}} \\
& \quad \text { if } t_{\text {reg }} \cdot L(L+1) \leq 1 \\
& \Delta t \leq \frac{h^{2}}{4 D_{11}+2 D_{33}+D_{44} h^{2} \frac{1}{2 e_{\text {reg }}}} \quad \text { if } t_{\text {reg }} \cdot L(L+1)>1
\end{aligned}
$$

where $h$ denotes spatial step size and $L=\left\lfloor\sqrt{n_{S H}-1}\right\rfloor$. The estimate (89) is due to the wellknown Gerschgorin circle theorem, cf. Gerschgorin (1931). An increase of spatial diffusivity and angular diffusivity yields a decrease in the maximum time step, whereas an increase of the regularity parameter $t_{\text {reg }}$ allows a larger time step. We also recognize a turning point if $t_{\text {reg }} \cdot(L(L+1))=1$. The multiplier $l(j)(l(j)+1) e^{-t_{\text {reg }} l(j)(l(j)+1)}$ attains its maximum at $j<n_{S H}$ if $t_{\text {reg }}>(L(L+1))^{-1}$. This is desirable since the multiplier is supposed to vanish at $j=n_{S H} \approx N_{o}$. In the experiment of Fig. 10, we have set $h=10^{-1}, n_{o}=162$, $L=18$ (restricting $l$ to even order), $t_{\text {reg }}=0.01, D_{33}=1$, $D_{11}=10^{-2}, D_{44}=10^{-4}$ and $\Delta t=0.005$.

\subsection{Convolution Schemes for Linear $\mathbb{R}^{3} \rtimes S^{2}$-Diffusion}

Instead of a finite difference scheme one can use the theoretical fact that the solutions of the linear diffusions, (36), are given by $\mathbb{R}^{3} \rtimes S^{2}$-convolution, (37), with the corresponding Green's function $p_{t}^{\mathbf{D}, \mathbf{a}}$ that we derived analytically in Sect. 6 . The convolution scheme is a relatively straightforward discretization of $W(\mathbf{y}, \mathbf{n}, t)=\left(p_{t}^{\mathbf{D}, \mathbf{a}} *_{\mathbb{R}^{3} \rtimes S^{2}} U\right)(\mathbf{y}, \mathbf{n})$ given by (10), where the integrals are usually replaced by sums using the mid-point rule (unless one has to deal with the singularity at the origin of the contour-completion kernel). We will consider specific practical implementation issues later in Sect. 8.2. In this subsection we restrict ourselves to an overview of options for the computation of the Green's functions.

We propose the following options to evaluate the Green's function for contour enhancement (i.e. non-zero parameters are $D_{33}>0, D_{44}=D_{55}>0$ and $\left.D_{11}=D_{22} \geq 0\right)$ in (37):

1. Use the finite difference scheme to numerically approximate the Green's function. Disadvantage: This requires interpolation. For small time steps $\Delta t \ll 1$ this numeric approximation is very accurate.

2. if $D_{11}=D_{22}=0$ we can use the analytic approximation formulae for the contour enhancement kernel. Here one can either use (75) where the functions $(\mathbf{x}, \mathbf{n}) \mapsto c^{k}(\mathbf{x}, \mathbf{n})$ are computed by means of the algorithm (76), or one may use the simpler but less accurate formula (69) using the asymptotical formula (70). In case $D_{44} / D_{33} \ll 1$ one may want to use the fast Heisenberg approximation kernel, (68), together with (70).

For the contour-completion case where the non-zero parameters are $a^{3}=1, D_{44}=D_{55}>0$ generalizations of the finite difference scheme of the previous section are questionable due to the trade-off between accuracy of convection and stability of diffusion. Alternation of convection and diffusion with very small time steps (like described in Zweck (2004) for the SE(2)-case) is probably preferable here. To avoid these technical issues we propose kernelimplementations for the contour completion case, where we distinguish between the following options:

1. For the resolvent of the contour-completion process use analytic formula (64) (accurate if $4 \lambda D_{44} \ll 1$ ).

2 . For the time-dependent contour completion process use analytical formula (65) (accurate if $4 t D_{44} \ll 1$ ).

Figure 7 shows HARDI glyph visualizations of several contour enhancement kernels and Fig. 8 shows HARDI glyph visualizations of a contour completion kernel.

\section{Experiments of Linear Crossing-preserving Diffusion on $\mathbb{R}^{3} \rtimes S^{2}$}

In the previous section we have discussed two different kinds of implementations of crossing-preserving diffusion on HARDI images, namely left-invariance finite difference schemes and left-invariant convolution schemes. In this section we will show some experiments of these approaches and furthermore we discuss some practical issues that come along with these approaches.

Before we will consider the different practical properties of the two approaches, we briefly comment on their analogies. Firstly, each step in the finite difference schemes is a linear kernel operator and thereby in principle (due to Corollary 1) a $\mathbb{R}^{3} \rtimes S^{2}$-convolution with a small kernel which is non-zero on the discretization stencil). Secondly, the computational order of the algorithms is comparable. The $\mathbb{R}^{3} \rtimes S^{2}$ convolutions are of order $O\left(N_{S} N_{o} K_{S} K_{o}\right)$ and the finite difference schemes are of order $O\left(N_{S} N_{o} N_{i t} N_{s t}\right)$, where $N_{s}, N_{o}$ respectively stands for the total number of spatial and orientation samples of the HARDI image, $K_{s}, K_{o}$ respectively stand for the number of spatial samples and average number of orientation samples of the convolution kernel and $N_{i t}$ stands for number of iteration with a discretization stencil of length $N_{s t}$. Thirdly, both approaches are very well suited for parallel implementation. 


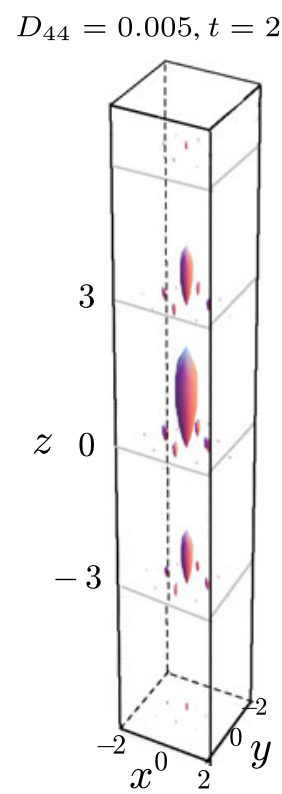

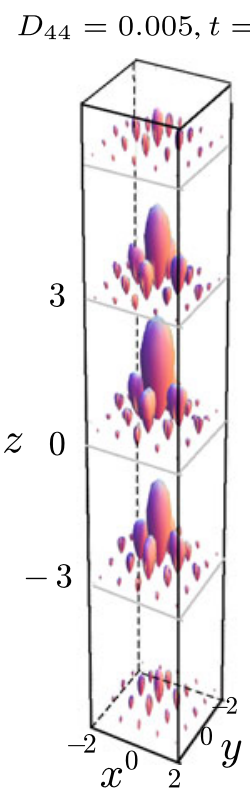

Fig. 7 Glyph visualization of the analytic approximations (for $0<$ $D_{44} / D_{33} \ll 1$ ), (69), using asymptotical formula (70) of the Green's function $p_{t}^{D_{33}, D_{44} ; \mathbb{R}^{3} \rtimes S^{2}}$ for contour enhancement, satisfying the semigroup property: $p_{t+s}^{D_{33}, D_{44} ; \mathbb{R}^{3} \rtimes S^{2}}=p_{s}^{D_{33}, D_{44} ; \mathbb{R}^{3} \rtimes S^{2}}{* \mathbb{R}^{3} \rtimes S^{2}}_{p_{t}}^{D_{33}, D_{44} ; \mathbb{R}^{3} \rtimes S^{2}}$
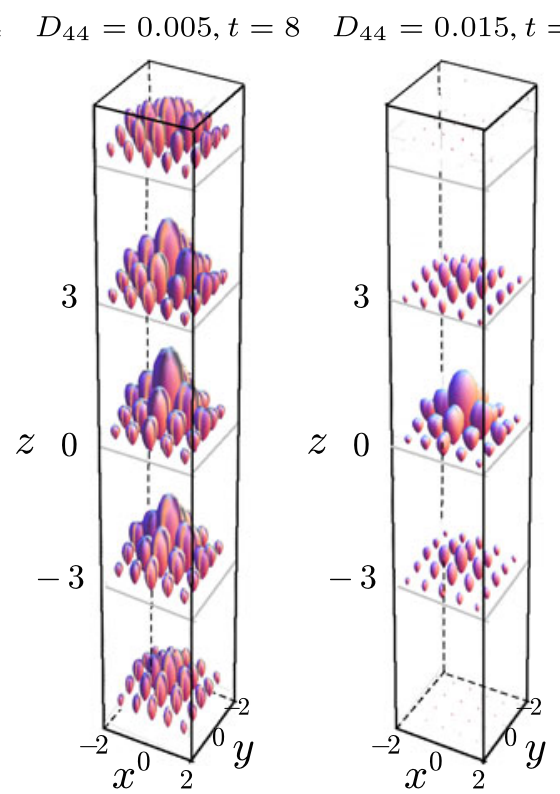

and consequently there arise no artefacts (such as in Fig. 5) in the iterative diffusion. We normalized stretching parameter $D_{33}=1$ and the values of $D_{44}$ and $t$ are depicted on top. The size of the kernel is controlled by $t>0$ and $D_{44}$ controls the bending of the kernel

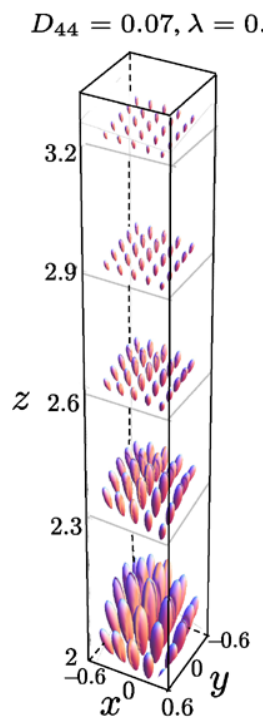

Fig. 8 Left: Glyph visualization of the analytic approximations (accurate for $0<4 \lambda D_{44} \ll 1$ ) given by (64) of the Green's function $p^{\lambda, D_{44} ; \mathbb{R}^{3} \rtimes S^{2}}$ for contour completion. Top right: HARDI glyphs at $(0,0, z)$ with from left to right $z=0.1,0.5,1,1.5$. The contour completion kernel is single-sided (i.e. $p^{\lambda, D_{44} ; \mathbb{R}^{3} \rtimes S^{2}}(x, y, z, \mathbf{n})=0$ for
Singularity at $z=x=y=0$

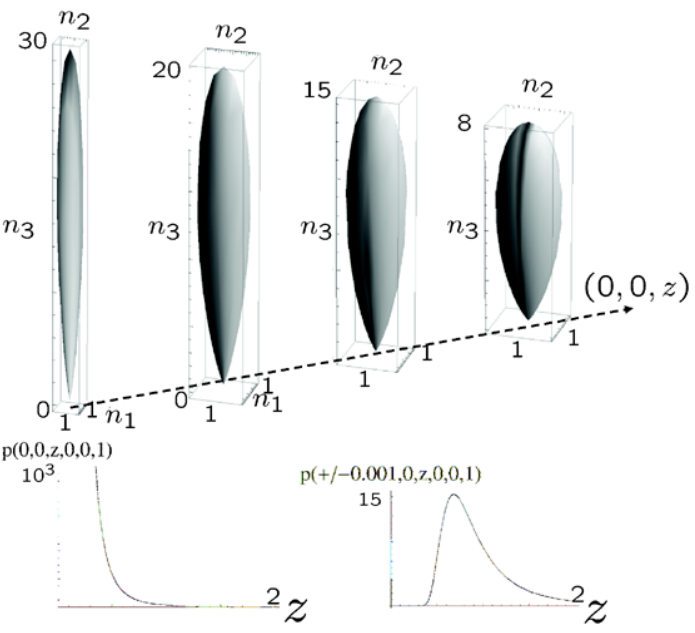

\subsection{Experiments Finite Difference Scheme}

We implemented linear, left-invariant diffusion on HARDI data with diagonal diffusion matrix $\mathbf{D}=\operatorname{diag}\left(D_{i i}\right)$ with $D_{11}=D_{22}, D_{44}=D_{55}\left(\right.$ and $\left.D_{66}=0\right)$ using an explicit nu- $z<0$ ), in contrast to the contour enhancement kernel depicted in Fig. 7. The positive probability density kernel $p^{\lambda, D_{44} ; \mathbb{R}^{3} \rtimes S^{2}}$ is $\mathbb{L}_{1}$-normalized but has a singularity at the origin, akin to its $2 \mathrm{D}$-equivalent (Duits and van Almsick 2008; Mumford 1994)

merical scheme as explained in Sect. 7.2. Figures 9 and 10 show results of the linear diffusion process. In these examples an artificial three-dimensional HARDI dataset is created, to which Rician noise is added, meaning that we applied the transformation 
Fig. 9 Result of

$\mathbb{R}^{3} \rtimes S^{2}$-diffusion (computed by the finite difference scheme,

Sect. 7.2) on an artificial

HARDI dataset of two crossing straight lines, with and without added Rician noise (90) with $\sigma=0.17$ (signal amplitude 1). Image size: $10 \times 10 \times 10$ spatial and 162 orientations. Parameters of the isotropic diffusion process: $D_{11}=D_{22}=D_{33}=1$, $D_{44}=D_{55}=0.01$. Parameters of the anisotropic diffusion process: $D_{11}=D_{22}=0.01$, $D_{33}=1, D_{44}=D_{55}=10^{-4}$. In both cases we have set $t_{\text {reg }}=0.01$ in (81)

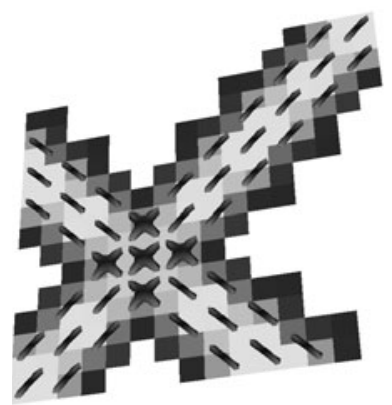

(a) $t=0$, no noise

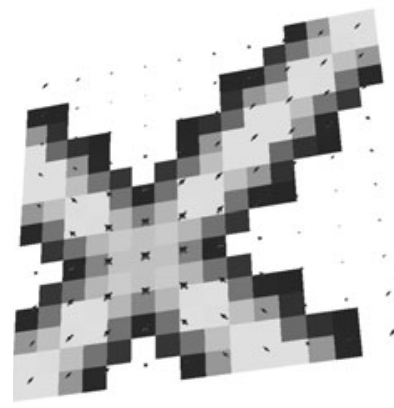

(c) $t=1, \mu$-isotropic, no noise

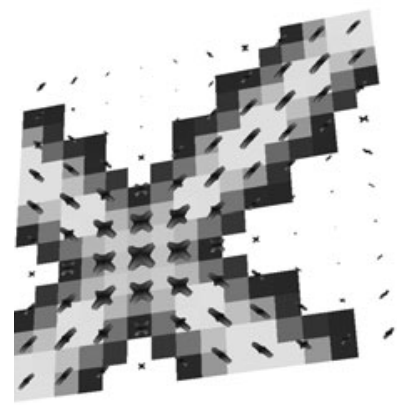

(e) $t=1$, anisotropic, no noise

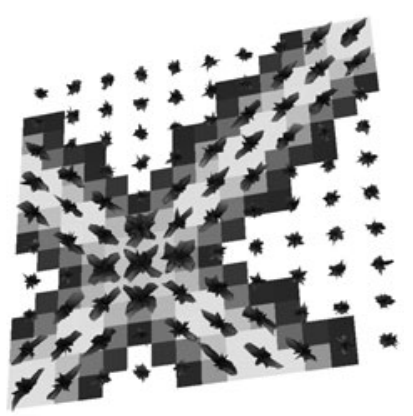

(b) $t=0$, added noise

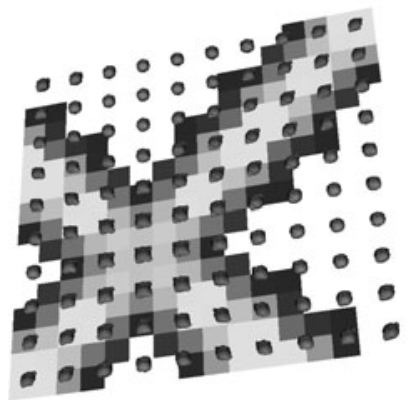

(d) $t=1, \mu$-isotropic, added noise

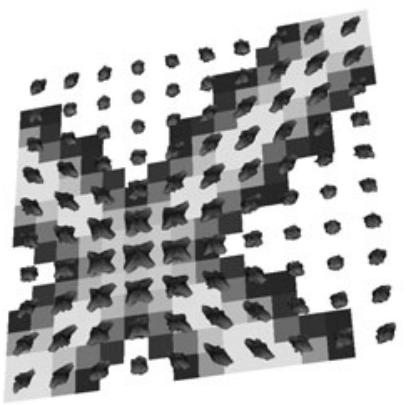

(f) $t=1$, anisotropic, added noise

$$
\begin{aligned}
& ((\mathbf{y}, \mathbf{n}) \mapsto U(\mathbf{y}, \mathbf{n})) \mapsto \\
& ((\mathbf{y}, \mathbf{n}) \mapsto \\
& \left.\quad \sqrt{\left(U(\mathbf{y}, \mathbf{n}) \cos \left(\eta_{1}\right)+\eta_{2}\right)^{2}+\left(U(\mathbf{y}, \mathbf{n}) \sin \left(\eta_{1}\right)+\eta_{3}\right)^{2}}\right)
\end{aligned}
$$

where $\eta_{2}, \eta_{3} \sim \mathcal{N}(0, \sigma)$ normally distributed and $\eta_{1}$ uniformly distributed over $[0,2 \pi)$.

Next, we applied two different $\mathbb{R}^{3} \rtimes S^{2}$-diffusions on both the noise-free and the noisy dataset. To visualize our results we used the DTI tool (see http://www.bmia.bmt.tue.nl/ software/dtitool/) which can visualize HARDI glyphs using the Q-ball visualization method (Descoteaux et al. 2007). In the results, all glyphs are scaled equivalently. The isotropic diffusion $\left(D_{33}=D_{22}=D_{11}\right)$ does not preserve the anisotropy of the glyphs well; especially in the noisy case we observe that we get almost isotropic glyphs.
With anisotropic diffusion, the anisotropy of the HARDI glyphs is preserved much better and in the noisy case the noise is clearly reduced. See Figures 9 and 10. The resulting glyphs are, however, less directed than in the noise-free input image. This would improve when using nonlinear diffusion. The basic theoretical PDE-framework for nonlinear diffusions, is the subject of the last section. As an alternative to nonlinear adaptive diffusion, we are currently investigating the inclusion of "sharpening" steps by means of left-invariant erosions (solutions of left-invariant HamiltonJacobi PDEs on HARDI data). Practical properties of the left-invariant finite difference schemes are:

++ It is relatively easy to adapt and generalize to nonlinear (adaptive) diffusion schemes.

- - The explicit finite difference scheme is only stable for sufficiently small time steps. 
Fig. 10 Result of

$\mathbb{R}^{3} \rtimes S^{2}$-diffusion (computed by the finite difference scheme, Sect. 7.2) on an artificial HARDI dataset of two crossing lines where one of the lines is curved, with and without added Rician noise, see (90), with $\sigma=0.17$ (signal amplitude 1). We have magnified the crossing areas on the top-right of the figures before and after the linear anisotropic diffusion. Image size: $10 \times 10 \times 10$ spatial and 162 orientations.

Parameters of the linear anisotropic diffusion process: $D_{11}=D_{22}=0.01, D_{33}=1$, $D_{44}=D_{55}=10^{-4}, t_{\text {reg }}=0.01$

in (81)

- The algorithm includes extra numerical blur, which does not accurately follow the continuous PDEtheory.

- If the DSHT-coefficients of the HARDI image quickly decay, best results are obtained with small $t_{\text {reg }}>0$. But often for those values of $t_{\text {reg }}$ the corresponding impulse response contains aliasing artefacts.

\subsection{Implementation and Experiments Convolution} Schemes

In Sect. 7.3 we have provided an overview of options for computing the Green's functions of contour enhancement and contour completion. Now suppose we have chosen an analytic approximation formula $p(\mathbf{y}, \mathbf{n})$ for the Green's function $p: \mathbb{R}^{3} \rtimes S^{2} \rightarrow \mathbb{R}^{+}$, then we can rewrite (37) in $\mathbb{L}_{2^{-}}$ inner product form

$\left(p *_{\mathbb{R}^{3} \rtimes S^{2}} U\right)(\mathbf{y}, \mathbf{n})=\left(\mathfrak{L}_{\left(\mathbf{y}, R_{\mathbf{n}}\right)} \check{p}, U\right)_{\mathbb{L}_{2}\left(\mathbb{R}^{3} \rtimes S^{2}\right)}$,

where we recall Definition 2 of $\mathfrak{L}$ and where we define $\check{p}\left(\mathbf{y}^{\prime}, \mathbf{n}^{\prime}\right):=p\left(-R_{\mathbf{n}^{\prime}}^{T} \mathbf{y}^{\prime}, R_{\mathbf{n}^{\prime}}^{T} \mathbf{e}_{z}\right)$. Note that

$\check{p}\left(\mathbf{y}^{\prime}, \mathbf{n}^{\prime}\right)=k\left(\mathbf{0}, \mathbf{e}_{z} ; \mathbf{y}^{\prime}, \mathbf{n}^{\prime}\right) \quad$ and

$p(\mathbf{y}, \mathbf{n})=k\left(\mathbf{y}, \mathbf{n} ; \mathbf{0}, \mathbf{e}_{z}\right), \quad$ for all $(\mathbf{y}, \mathbf{n}),\left(\mathbf{y}^{\prime}, \mathbf{n}^{\prime}\right) \in \mathbb{R}^{3} \rtimes S^{2}$.

Now $p(\mathbf{y}, \mathbf{n})$ denotes the probability density of finding a random walker at $(\mathbf{y}, \mathbf{n})$ given that it started at $\left(\mathbf{0}, \mathbf{e}_{z}\right)$, so that $\check{p}(\mathbf{y}, \mathbf{n})=p\left(-R_{\mathbf{n}}^{T} \mathbf{y}, R_{\mathbf{n}}^{T} \mathbf{e}_{z}\right)$ denotes the probability density of finding a random walker at $\left(-R_{\mathbf{n}}^{T} \mathbf{y}, R_{\mathbf{n}}^{T} \mathbf{e}_{z}\right)$ given that it started at $\left(\mathbf{0}, \mathbf{e}_{z}\right)$, which is by left-invariance of the stochastic process the same as the probability density of finding a random walker at $\left(\mathbf{0}, \mathbf{e}_{z}\right)$ given that it started at $(\mathbf{y}, \mathbf{n})$.

The main advantage of formula (91) is that in practice we can pre-compute/sample all rotated versions and translated versions $\left\{\mathfrak{L}_{\left(\mathbf{y}, R_{\mathbf{n}}\right)} \check{p} \mid \mathbf{y} \in \mathbb{R}^{3}, \mathbf{n} \in S^{2}\right\}$ of the check-kernel $\check{p}$, so that the remainder of the algorithm just consists of computing $\mathbb{L}_{2}$-inner products which can be done in parallel. In contrast to the finite difference schemes, the convolution schemes are unconditionally stable. In fact, we even have

$$
\begin{aligned}
\| p & *_{\mathbb{R}^{3} \rtimes S^{2}} U \|_{\mathbb{L}_{1}\left(\mathbb{R}^{3} \rtimes S^{2}\right)} \\
= & \|p\|_{\mathbb{L}_{1}\left(\mathbb{R}^{3} \rtimes S^{2}\right)}\|U\|_{\mathbb{L}_{1}\left(\mathbb{R}^{3} \rtimes S^{2}\right)} \\
& =1 \cdot\|U\|_{\mathbb{L}_{1}\left(\mathbb{R}^{3} \rtimes S^{2}\right)}, \\
\| p & *_{\mathbb{R}^{3} \rtimes S^{2}} U \|_{\mathbb{L}_{\infty}\left(\mathbb{R}^{3} \rtimes S^{2}\right)} \\
= & \sup _{(\mathbf{y}, \mathbf{n})}\left|p *_{\mathbb{R}^{3} \rtimes S^{2}} U(\mathbf{y}, \mathbf{n})\right| \\
\leq & \left\|\mathfrak{L}_{\left(\mathbf{y}, R_{\mathbf{n}}\right)} \check{p}\right\|_{\mathbb{L}_{2}\left(\mathbb{R}^{3} \rtimes S^{2}\right)}\|U\|_{\mathbb{L}_{2}\left(\mathbb{R}^{3} \rtimes S^{2}\right)} \\
\leq & \|p\|_{\mathbb{L}_{2}\left(\mathbb{R}^{3} \rtimes S^{2}\right)}\|U\|_{\mathbb{L}_{2}\left(\mathbb{R}^{3} \rtimes S^{2}\right)}
\end{aligned}
$$

i.e. preservation of mass (which also holds on the discrete grid if the discretely sampled versions of $\mathfrak{L}_{\left(\mathbf{y}, R_{\mathbf{n}}\right)} \breve{p}$ are 

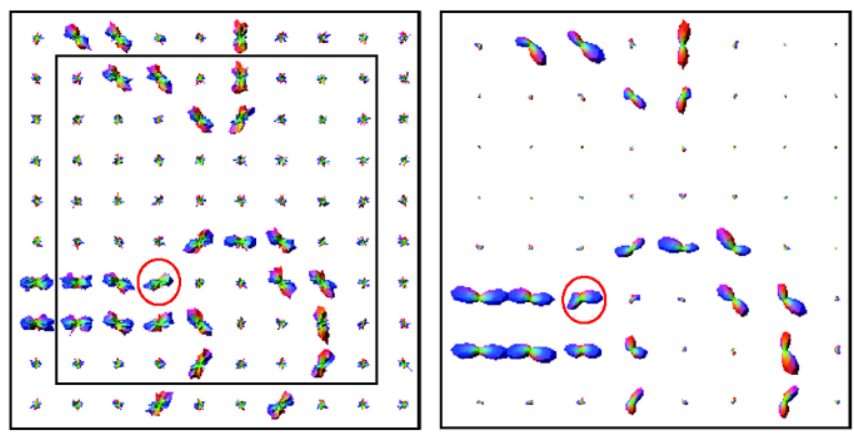

Fig. 11 Left: Plane in artificial 3D DTI input data (outside the plane the DTI tensor field is set to 0$)$. We added Rician noise, (90), $\sigma=0.2$. Right: squared output of convolution algorithm (96) and (92) (with $R=1$, tol $=10^{-5}$ ) applying respectively, from left to right, a contour

$\ell_{1}$-normalized on the grid) and a small $\mathbb{L}_{2}$-perturbation on the input yields a small $\mathbb{L}_{\infty}$-perturbation on the output.

Finally, formula (91) provides the following fast discrete approximation/truncation:

$$
\begin{aligned}
& \left(p *_{\mathbb{R}^{3} \rtimes S^{2}} U\right)(\mathbf{y}, \mathbf{n}) \\
& \approx \sum_{\mathbf{y}^{\prime} \in \mathbb{R}^{3},\left\|\mathbf{y}^{\prime}-\mathbf{y}\right\|_{\infty} \leq R} \sum_{k=1}^{K\left(\mathbf{y}^{\prime}, \mathbf{y}, \mathbf{n}\right)}\left(\mathfrak{L}_{\mathbf{y}, R_{\mathbf{n}}} \check{p}\right)\left(\mathbf{y}^{\prime}, \mathbf{n}_{k}^{\prime}\right) \\
& \quad \cdot U\left(\mathbf{y}^{\prime}, \mathbf{n}_{k}^{\prime}\right) \Delta \mathbf{y}^{\prime} \delta_{S^{2}}\left(\mathbf{n}_{k}^{\prime}\right),
\end{aligned}
$$

with spatial step size $\Delta \mathbf{y}=\left(\Delta y_{1}^{\prime}, \Delta y_{2}^{\prime}, \Delta y_{3}^{\prime}\right)$ and where we truncated the spatial integration to a cube $\left\|\mathbf{y}^{\prime}-\mathbf{y}\right\|_{\infty}=$ $\sup _{i=1,2,3}\left|y_{i}^{\prime}-y_{i}\right| \leq R, R>0$, where we recall (83) and where $\left\{\mathbf{n}_{k}^{\prime}\right\}_{k=1}^{N_{o}}:=\left\{\mathbf{n}_{k}^{\prime}\left(\mathbf{y}^{\prime}, \mathbf{y}, \mathbf{n}\right)\right\}_{k=1}^{N_{o}}$, forms an a priori defined lookup table by sorting the points $\left\{\mathbf{n}_{k}^{\prime}\right\}_{k=1}^{N_{o}}$ such that

$\mathfrak{L}_{\left(\mathbf{y}, R_{\mathbf{n}}\right)} \check{p}\left(\mathbf{y}^{\prime}, \mathbf{n}_{k+1}^{\prime}\right)<\mathfrak{L}_{\left(\mathbf{y}, R_{\mathbf{n}}\right)} \check{p}\left(\mathbf{y}^{\prime}, \mathbf{n}_{k}^{\prime}\right)$

and where $K\left(\mathbf{y}^{\prime}, \mathbf{y}, \mathbf{n}\right)=\max \left\{k \in\left\{1, \ldots, N_{o}\right\} \quad\right.$ | $\left.\mathfrak{L}_{\left(\mathbf{y}, R_{\mathbf{n}}\right)} \breve{p}\left(\mathbf{y}^{\prime}, \mathbf{n}_{k}\right) \leq \epsilon\right\}$, with tolerance $\epsilon>0$. We usually set $R \in\{1,2,3,4\}$ (see for example Fig. 11 where we even set $R=1$ ) for the spatial truncation and $\epsilon=10^{-5}$ for angular truncation. The gain in speed mostly lies in the angular truncation, as the convolution kernels are for reasonable parameter settings of $D_{33}, D_{44}, t, \lambda$ rather orientation-selective, recall Fig. 7 and Fig. 8.

Summarizing we have the following practical properties of the left-invariant convolution schemes:

$+\quad$ The convolution kernels can be pre-computed and truncated.

+ The algorithm does not suffer from the typical numerical blur of finite difference schemes.

++ The algorithm is unconditionally stable.
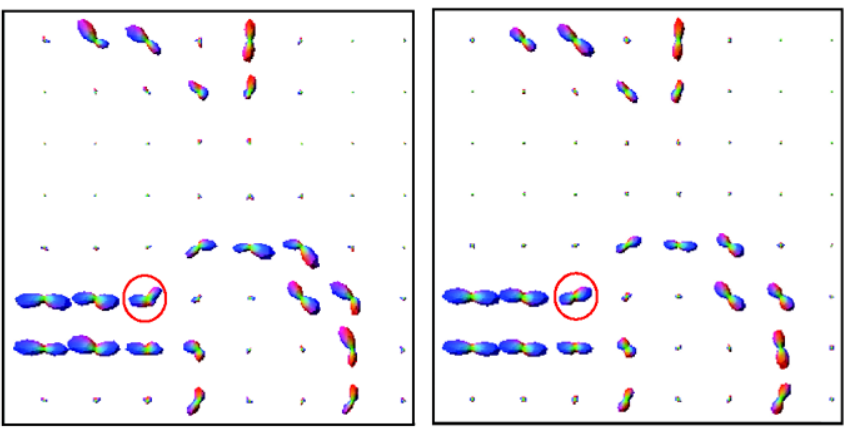

completion kernel ( $a=1, b=0)$, a spatially reflected completion kernel $(a=0, b=1)$, and the sum of a reflected and non-reflected completion kernel $(a=1, b=1)$. All kernels are sampled on a $3 \times 3 \times 3 \times 162$ grid, whereas input (left) is sampled on a $10 \times 10 \times 10 \times 162$-grid

$+\quad$ For single sided kernels (completion) one can include reflections, as we will see in Sect. 8.2.1.

++ The algorithm is easily extended to left-invariant dilations/erosions on HARDI images by replacing the $(+, \cdot)$-algebra by the $(\max ,+)$-algebra in the convolution, akin to previous work on regular images (Burgeth and Weickert 2003; Burgeth et al. 2008).

-- Generalization to nonlinear adaptive diffusions: Adapting the kernel to the data locally is no longer a convolution and the relation to left-invariant PDEs is no longer clear.

For further experiments of convolution schemes solving diffusion (combined with basic grey-value transformations, such as squaring $\left.U(\mathbf{y}, \mathbf{n}) \mapsto(U(\mathbf{y}, \mathbf{n}))^{2}\right)$ on medical HARDI and DTI datasets, see the recent works (Rodrigues et al. 2010; Prčkovska et al. 2010), where the first author of this article collaborated with P. Rodrigues, V. Prčkovska et al.

\subsubsection{Spherical Reflection Symmetries: Preservation of Reflection Symmetry in Contour Enhancement and including Glyph-attraction in Contour Completion}

The big difference between the stochastic processes for contour completion and contour enhancement is that contour completion kernels are single-sided, whereas contour enhancement kernels are double-sided. Compare Fig. 7 to Fig. 8. This change in reflection symmetry has two consequences:

1. The initial HARDI data are usually invariant under spherical reflections, i.e. $U(\mathbf{y}, \mathbf{n})=\left(\mathfrak{r}_{S^{2}} U\right)(\mathbf{y}, \mathbf{n}):=U(\mathbf{y},-\mathbf{n})$. In contrast to contour completion, contour enhancement preserves this symmetry during the evolution, as can be seen in Fig. 9 and Fig. 10. This directly follows by the fact that the generator of contour completion $-\mathcal{A}_{3}+\Delta_{S^{2}}$ 
and the generator of contour enhancement $\left(\mathcal{A}_{3}\right)^{2}+\Delta_{S^{2}}$ satisfy

$$
\begin{aligned}
& \left(-\mathcal{A}_{3}+\Delta_{S^{2}}\right) \circ \mathfrak{r}_{S^{2}}=\mathfrak{r}_{S^{2}} \circ\left(+\mathcal{A}_{3}+\Delta_{S^{2}}\right), \\
& \left(\left(\mathcal{A}_{3}\right)^{2}+\Delta_{S^{2}}\right) \circ \mathfrak{r}_{S^{2}}=\mathfrak{r}_{S^{2}} \circ\left(\left(\mathcal{A}_{3}\right)^{2}+\Delta_{S^{2}}\right) .
\end{aligned}
$$

2. It allows a relevant extension of our contour completion processes, where we replace the $\mathbb{R}^{3} \rtimes S^{2}$-convolutions by $\left(\mathbb{R}^{3} \rtimes O(3)\right) /(\{\mathbf{0}\} \times S O(2))$ convolutions as we will explain next. Here $O(3)=\left\{M \in \mathbb{R}^{3 \times 3} \mid M^{T}=M^{-1}\right\}$ denotes the group of orthogonal matrices on $\mathbb{R}^{3}$ which includes both rotations $\operatorname{det} M=+1$ and reflections $\operatorname{det} M=-1$. The practical advantage of these $\left(\mathbb{R}^{3} \rtimes O(3)\right) /(\{\mathbf{0}\} \times S O(2))$-convolution is that it allows us to include attraction of glyphs, rather than continuation of glyphs. See Fig. 11 and compare the two images in the middle. To achieve this extension we need a different kind of reflections, namely spatial reflections given by $\mathfrak{r}_{\mathbb{R}^{3}} U(\mathbf{y}, \mathbf{n}):=U(-\mathbf{y}, \mathbf{n})$. These spatial reflections $\mathfrak{r}_{\mathbb{R}^{2}}$ intertwine the contour enhancement and completion generators in the same way as the angular reflections $\mathfrak{r}_{S^{2}}$ :

$$
\begin{aligned}
& \left(-\mathcal{A}_{3}+\Delta_{S^{2}}\right) \circ \mathfrak{r}_{\mathbb{R}^{3}}=\mathfrak{r}_{\mathbb{R}^{3}} \circ\left(+\mathcal{A}_{3}+\Delta_{S^{2}}\right), \\
& \left(\left(\mathcal{A}_{3}\right)^{2}+\Delta_{S^{2}}\right) \circ \mathfrak{r}_{\mathbb{R}^{3}}=\mathfrak{r}_{\mathbb{R}^{3}} \circ\left(\left(\mathcal{A}_{3}\right)^{2}+\Delta_{S^{2}}\right) .
\end{aligned}
$$

Now indeed (94) is analogous to (93) but the crucial difference between these two types of reflections is:

$$
\begin{aligned}
& \mathfrak{r}_{\mathbb{R}^{3} \delta_{e}=\delta_{e}} \quad \text { and } \quad \mathfrak{r}_{S^{2}} \delta_{e} \neq \delta_{e} \quad \text { and } \\
& \mathfrak{r}_{\mathbb{R}^{3}} U \neq U \quad \text { and } \quad \mathfrak{r}_{S^{2}} U=U .
\end{aligned}
$$

Now (95) and (94) directly imply the spatial reflection symmetry of the contour enhancement kernel as can be seen in Fig. 7. Clearly the contour completion kernel does not admit such a symmetry and we arrive at the following three possible choices of $\left(\mathbb{R}^{3} \rtimes O(3)\right) /(\{\mathbf{0}\} \times S O(2))$ convolutions:

$$
\begin{aligned}
& \left(p^{\lambda, D_{44}} *_{\left(\mathbb{R}^{3} \rtimes O(3)\right) /(\{\mathbf{0}\} \times S O(2))} U\right)(\mathbf{y}, \mathbf{n}) \\
& =\sum_{\varepsilon^{\prime} \in\{-1,1\}} q_{a b}\left(\varepsilon^{\prime}\right) \int_{S^{2}} \int_{\mathbb{R}^{3}} p^{\lambda, D_{44}}\left(\varepsilon^{\prime} R_{\mathbf{n}^{\prime}}^{T}\left(\mathbf{y}-\mathbf{y}^{\prime}\right), R_{\mathbf{n}^{\prime}}^{T} \mathbf{n}\right) \\
& \quad \cdot U\left(\mathbf{y}^{\prime}, \mathbf{n}^{\prime}\right) \mathrm{d} \mathbf{y}^{\prime} \mathrm{d} \sigma\left(\mathbf{n}^{\prime}\right)
\end{aligned}
$$$$
\text { with } q_{a b}\left(\varepsilon^{\prime}\right)=\frac{1}{2}\left(a \delta_{\varepsilon^{\prime} 1}+b \delta_{-\varepsilon^{\prime} 1}\right) \text {, }
$$$$
a, b \in\{0,1\}, a b \neq 0 \text {. }
$$

These two issues are illustrated in Fig. 11 for a simple example of a HARDI image induced by a DTI image, i.e. $U(\mathbf{y}, \mathbf{n})=\mathbf{n}^{T} D(\mathbf{y}) \mathbf{n}$. In Fig. 11 we have set rotation matrices $S(\mathbf{y})$ such that $D(\mathbf{y})=(S(\mathbf{y}))^{-1} \operatorname{diag}\{0.1,0.1,1\} S(\mathbf{y})$. Furthermore, we applied Rician noise on the HARDI data, recall (90). The particular case $a=b=1$ in (96) yields results that are similar to convolution with contour enhancement kernels (recall Fig. 7) for suitable choice of $D_{33}$ and $D_{44}$. The difference in practice is that the sum of two spatially reflected contour completion kernels yields a double sided kernel that is typically sharper kernel at the center than a contour enhancement kernel.

\section{Nonlinear, Adaptive, Left-Invariant Diffusions on HARDI Images}

So far we have considered linear left-invariant diffusions, whose solutions are given by convolution with a fixed Green's function reflecting an a priori probability model for fiber-extension. In many applications however, it is important to adapt the fiber-extension model to the data, where we can include adaptive curvature and adaptive torsion. Now by Theorem 3, it follows that in order to include adaptive torsion and curvature we must re-align the left-invariant local coordinate frame $\left\{\mathcal{A}_{1}, \ldots, \mathcal{A}_{5}\right\}$ by means of a locally optimally fitting exponential curve, where we recall (51).

Our first aim is to determine the exponential curve, recall (54), that optimally fits the distribution $(\mathbf{y}, \mathbf{n}) \mapsto$ $U(\mathbf{y}, \mathbf{n}) \in \mathbb{R}^{+}$at each position $(\mathbf{y}, \mathbf{n}) \in \mathbb{R}^{3} \rtimes S^{2}$. Recall that such a distribution gives rise to a probability distribution $(\mathbf{x}, R) \mapsto \tilde{U}(\mathbf{x}, R)$ on $S E(3)$ by means of (27). To achieve our goal, we follow the same approach as in our previous works on nonlinear diffusions on invertible orientation scores (of 2D-images) defined on $S E(2)$ (Franken 2008, pp. 118-120), (Duits and Franken 2010, Part II, Chap. 3.4), (Franken and Duits 2009, Chap. 5.2). We again formulate a minimization problem that minimizes over the "iso-contours" of the left-invariant gradient vector at $(\mathbf{y}, \mathbf{n}) \in \mathbb{R}^{3} \rtimes S^{2}$, yielding optimal tangent vector $\mathbf{c}_{*}(\mathbf{y}, \mathbf{n})=\left(c_{*}^{1}(\mathbf{y}, \mathbf{n}), \ldots, c_{*}^{5}(\mathbf{y}, \mathbf{n}), 0\right)^{T}:$

$$
\begin{aligned}
\mathbf{c}_{*}(\mathbf{y}, \mathbf{n})= & \arg \min _{\mathbf{c}(\mathbf{y}, \mathbf{n})}\left\{\left\|\left.\frac{\mathrm{d}}{\mathrm{d} t}\left(\nabla \tilde{U}\left(g e^{t \mathbf{c}(\mathbf{y}, \mathbf{n})}\right)\right)\right|_{t=0}\right\|_{\mu}^{2} \mid\right. \\
& \left.\|\mathbf{c}(\mathbf{y}, \mathbf{n})\|_{\mu}=1\right\},
\end{aligned}
$$

where the left-invariant gradient

$\mathrm{d} \tilde{U}(g)=\left.\sum_{i=1}^{5}\left(\mathcal{A}_{i}(\tilde{U})\right)(g) \mathrm{d} \mathcal{A}^{i}\right|_{g}, \quad g \in \operatorname{SE}(3)$,

a co-vector field, is represented by a row-vector given by $\nabla \tilde{U}(g)=\left(\mathcal{A}_{1} \tilde{U}(g), \ldots, \mathcal{A}_{5} \tilde{U}(g), 0\right)$ and where $\|\cdot\|_{\mu}$ denotes both the norm on a vector in tangent space $T_{g}(S E(3))$ and the dual norm on a covector in the dual tangent space $T_{g}^{*}(\operatorname{SE}(3))$. We represent tangent vectors $\mathbf{c}(\mathbf{y}, \mathbf{n})=$ $\left.\sum_{i=1}^{5} c^{i}(\mathbf{y}, \mathbf{n}) \mathcal{A}_{i}\right|_{g=\left(\mathbf{y}, R_{\mathbf{n}}\right)}$ as column-vector $\mathbf{c}(\mathbf{y}, \mathbf{n})=$ 
$\left(c_{*}^{1}(\mathbf{y}, \mathbf{n}), \ldots, c_{*}^{5}(\mathbf{y}, \mathbf{n}), 0\right)^{T}$ and their norm is defined by $\|\mathbf{c}\|_{\mu}:=\sqrt{(\mathbf{c}, \mathbf{c})_{\mu}}$ with the inner product $(\mathbf{c}, \mathbf{c})_{\mu}:=$ $\mu^{2}\left(\sum_{j=1}^{3} c^{j} c^{j}\right)+\sum_{j=4}^{6} c^{j} c^{j}$, where parameter ${ }^{10} \mu$ ensures that all components of the inner product are dimensionless. The value of the parameter determines how the distance in the spatial dimensions relates to distance in the orientation dimension. Implicitly, this also defines the norm on covectors by $\|\hat{\mathbf{c}}\|_{\mu}=\sqrt{(\hat{\mathbf{c}}, \hat{\mathbf{c}})_{\mu}},(\hat{\mathbf{c}}, \hat{\mathbf{c}})_{\mu}=\left\langle\hat{\mathbf{c}}, G_{\mu}^{-1} \hat{\mathbf{c}}\right\rangle=$ $\mu^{-2}\left(\sum_{j=1}^{3} c_{j} c_{j}\right)+\sum_{j=4}^{6} c_{j} c_{j}$. By means of the calculus of variations it follows that the minimizer $\mathbf{c}_{*}(\mathbf{y}, \mathbf{n})$ satisfies

$$
\left(M_{\mu} \mathcal{H} \tilde{U}(g) M_{\mu}\right)^{T}\left(M_{\mu} \mathcal{H} \tilde{U}(g) M_{\mu}\right) \tilde{\mathbf{c}}^{*}(\mathbf{y}, \mathbf{n})=\lambda \tilde{\mathbf{c}}^{*}(\mathbf{y}, \mathbf{n}),
$$

with $M_{\mu}:=\operatorname{diag}(1 / \mu, 1 / \mu, 1 / \mu, 1,1,1), \tilde{\mathbf{c}}_{*}=M_{\mu}^{-1} \mathbf{c}_{*}$ and where the $6 \times 6$ Hessian of $\tilde{U}$ on $\mathbb{R}^{3} \rtimes S^{2}$ equals

$$
\begin{aligned}
& \mathcal{H} \tilde{U}(g)=\left[\mathcal{A}_{j} \mathcal{A}_{i} U(g)\right]_{j}^{i} \text { row-index } \text { column index }, \\
& g=(\mathbf{y}, R) \in S E(3),
\end{aligned}
$$

where the last row contains of zeros only. This amounts to eigensystem analysis of the symmetric $6 \times 6$ matrixvalued function $g \mapsto\left(M_{\mu} \mathcal{H} \tilde{U}(g) M_{\mu}\right)^{T}\left(M_{\mu} \mathcal{H} \tilde{U}(g) M_{\mu}\right)$, where one of the three eigenvectors gives $\tilde{\mathbf{c}}_{*}(\mathbf{y}, \mathbf{n})$. The eigenvector with the smallest corresponding eigenvalue is selected as tangent vector $\tilde{\mathbf{c}}_{*}(\mathbf{y}, \mathbf{n})$, and the desired tangent vector $\mathbf{c}_{*}(\mathbf{y}, \mathbf{n})$ is then given by $\mathbf{c}_{*}(\mathbf{y}, \mathbf{n})=M_{\mu} \tilde{\mathbf{c}}_{*}(\mathbf{y}, \mathbf{n})$.

Now that we have computed the optimal tangent vector $\mathbf{c}(\mathbf{y}, \mathbf{n})$ at $(\mathbf{y}, \mathbf{n}) \mapsto U(\mathbf{y}, \mathbf{n})$ (and thereby the best fitting exponential curve $t \mapsto g e^{\sum_{i=1}^{5} c^{i} A_{i}}$ in $\left.\mathbb{R}^{3} \rtimes S^{2}\right)$ we construct the nonlinear adaptive diffusion function:

$$
\begin{aligned}
\mathbf{D}(U)(\mathbf{y}, \mathbf{n})= & \mathbf{c}_{*}(\mathbf{y}, \mathbf{n}) \mathbf{c}(\mathbf{y}, \mathbf{n})^{T} \frac{\mu^{2}\left(1-D_{a}(U)(\mathbf{y}, \mathbf{n})\right)}{\|\mathbf{c}(\mathbf{y}, \mathbf{n})\|_{\mu}^{2}} \\
& +D_{a}(U)(\mathbf{y}, \mathbf{n})\left(\begin{array}{cc}
I_{3} & \mathbf{0} \\
\mathbf{0} & \mu^{2} I_{3}
\end{array}\right),
\end{aligned}
$$

where $D_{a}(U)(\mathbf{y}, \mathbf{n})$ is a locally adaptive anisotropy factor. Finally, we note that the conditions (35) are satisfied so our final well-defined nonlinear diffusion system on the HARDI data are:

$$
\left\{\begin{array}{l}
\partial_{t} W(\mathbf{y}, \mathbf{n}, t)=\sum_{i, j=1}^{6}\left(\mathcal{A}_{i}[\mathbf{D}(U)(\mathbf{y}, \mathbf{n})]_{i j} \mathcal{A}_{j} W\right)(\mathbf{y}, \mathbf{n}, t), \\
\lim _{t \downarrow 0} W(\mathbf{y}, \mathbf{n}, t)=U(\mathbf{y}, \mathbf{n}) .
\end{array}\right.
$$

\footnotetext{
${ }^{10}$ In some of our previous works on $S E(2)$ we denoted this fundamental parameter by $\beta^{-1}>0$, but here we use $\mu>0$ to avoid confusion with Euler-angle $\beta>0$ in $S O(3)$. The left-invariant Riemannian metric on $S E(3)$ is given by $\sum_{i=1}^{3} \mu^{2} \mathrm{~d} \mathcal{A}^{i} \otimes \mathrm{d} \mathcal{A}^{i}+\sum_{j=4}^{6} \mathrm{~d} \mathcal{A}^{j} \otimes \mathrm{d} \mathcal{A}^{j}$.
}

\section{Conclusion}

For the purpose of tractography (detection of biological fibers) and visualization, DTI and HARDI data should be enhanced such that fiber junctions are maintained, while reducing high frequency noise in the joined domain $\mathbb{R}^{3} \rtimes S^{2}$ of positions and orientations. Therefore we have considered diffusions on HARDI and DTI induced by fundamental stochastic processes on $\mathbb{R}^{3} \rtimes S^{2}$ embedded in the group manifold $S E(3)$ of 3D rigid body motions.

We have shown that the processing must be left-invariant and we have classified all linear left-invariant diffusions on HARDI images. We presented two novel diffusion approaches which take place simultaneously over both positions and orientations. These two approaches do allow enhancement of fibres while preserving crossings and/or bifurcations. These two diffusions are Fokker-Planck equations of stochastic processes (random walks) for respectively contour enhancement and contour completion. In a contour completion process a random walker always proceeds forward in space along its prescribed random direction, whereas in a contour enhancement process the random walker randomly moves forward and backward in its prescribed random orientation. As a result the contour completion process is generated by $-\mathcal{A}_{3}+D_{44} \Delta_{S^{2}}$ whereas the contour enhancement process is generated by the subLaplacian $+D_{33}\left(\mathcal{A}_{3}\right)^{2}+D_{44} \Delta_{S^{2}}$, with $D_{33}, D_{44}>0$ and $\left.\Delta_{S^{2}}\right|_{H}=\mathcal{A}_{4}^{2}+\mathcal{A}_{5}^{2}$ the Laplace-Beltrami operator on the sphere and where $\mathcal{A}_{i}$-denotes the $i$-th left-invariant vector field on $\mathbb{R}^{3} \rtimes S^{2}$. Consequently, the contour enhancement process preserves the angular reflection symmetry of HARDI data, whereas the contour completion process allows a choice between attraction or continuation of glyphs.

As the solutions of linear left-invariant diffusion equations are given by $\mathbb{R}^{3} \rtimes S^{2}$-convolution with their Green's functions, we arrive at two types of implementations: Convolution schemes and finite difference schemes. Practical advantages of convolution schemes over finite difference schemes for linear diffusions are: they are unconditionally stable and do not involve the typical numerical blurring of a finite difference scheme. However, the finite difference schemes with sufficiently small time steps do provide crossing preserving diffusion as well, and they are preferable for our extensions to nonlinear adaptive diffusions proposed in Sect. 9.

The crucial theoretical observations in our framework lie in the fact that the left-invariant evolution equations are expressed by a quadratic form in the left-invariant vector fields $\left\{\mathcal{A}_{i}\right\}_{i=1}^{6}$ on $\mathbb{R}^{3} \rtimes S^{2}$ embedded in $S E(3)$, which form a moving frame of reference consisting of a spatial velocity part $\left\{\mathcal{A}_{1}, \mathcal{A}_{2}, \mathcal{A}_{3}\right\}$ and an angular velocity part $\left\{\mathcal{A}_{4}, \mathcal{A}_{5}, \mathcal{A}_{6}\right\}$. This moving frame of reference requires the Cartan connec- 
tion viewpoint ${ }^{11}$ on the underlying differential geometry and by expressing the left-invariant diffusions in covariant derivatives we see that even the adaptive nonlinear left-invariant evolutions locally take place along the covariantly constant curves, which coincide with the exponential curves in $S E(3)$. The spatial part of the exponential curves are circular spirals, i.e. curves in $\mathbb{R}^{3}$ with constant curvature and constant torsion. As a result our nonlinear adaptive diffusion schemes allow local adaptation for curvature and torsion, which we will further investigate and implement in future work.

Furthermore, in future work, we will apply our techniques to medical $D T I$-data sets and investigate whether we can create suitable orientation density distributions (to avoid expensive HARDI acquisitions) at crossings by means of $\mathbb{R}^{3} \rtimes S^{2}$-diffusion. Finally, we will consider natural extensions of our scale spaces on $\mathbb{R}^{3} \rtimes S^{2}$, such as the combination of left-invariant diffusion and left-invariant HamiltonJacobi equations (erosions, Burgeth and Weickert 2003) in a single evolution on $\mathbb{R}^{3} \rtimes S^{2}$.

Acknowledgements Vesna Prčkovska and Paulo Rodrigues, biomedical image analysis group Eindhoven University of Technology are gratefully acknowledged for providing the artificial HARDI test images and the DTI tool supporting HARDI glyphs. Special thanks to Mark Bruurmijn and Paulo Rodrigues, Eindhoven University of Technology, for their support on the visualization and implementation of $\mathbb{R}^{3} \rtimes S^{2}$-convolutions. The Netherlands Organization for Scientific Research (NWO) is gratefully acknowledged for financial support.

Open Access This article is distributed under the terms of the Creative Commons Attribution Noncommercial License which permits any noncommercial use, distribution, and reproduction in any medium, provided the original author(s) and source are credited.

\section{References}

Alvarez, L., Guichard, F., Lions, P. L., \& Morel, J.-M. (1993). Axioms and fundamental equations of image processing. Archive for $\mathrm{Ra}$ tional Mechanics and Analysis, 123, 200-257.

Arsigny, V., Pennec, X., \& Ayache, N. (2006). Bi-invariant means in Lie groups. Application to Left-invariant Polyaffine Transformations. Technical report, Nr. 5885, INRIA, France.

Aubin, T. (2001) Graduate studies in mathematics: Vol. 27. A course in differential geometry. Providence: AMS

Barmpoutis, A., Vemuri, B. C., Howland, D., \& Forder, J. R. (2008). Extracting tractosemas from a displacement probability field for tractography in DW-MRI. In Lecture notes in computer science: Vol. 5241. Proc. MICCAI 2008, Part I (pp. 9-16). Berlin: Springer.

Bukhvalov, A. V., \& Arendt, W. (1994). Integral representation of resolvent and semigroups. Forum Mathematicum, 6(1), 111-137.

Burgeth, B., \& Weickert, J. (2003). An explanation for the logarithmic connection between linear and morphological systems. In Lecture notes in computer science: Proc. 4th int. conference scale space 2003 (pp. 325-339). Berlin: Springer.

\footnotetext{
${ }^{11}$ Rather than the torsion free Levi-Civita connection on the Riemannian manifold $\left(\operatorname{SE}(3),\left(\operatorname{diag}\left(\mu^{2}, \mu^{2}, \mu^{2}, 1,1,1\right)\right)_{i j} \mathrm{~d} \mathcal{A}^{i} \otimes \mathrm{d} \mathcal{A}^{j}\right)$.
}

Burgeth, B., Breuss, M., Didas, S., \& Weickert, J. (2008). PDE-based morphology for matrix fields: numerical solution schemes. Technical Report No. 220, Math. and Comp. Science, Saarland University, Saarbrücken.

Chirikjian, G. S., \& Kyatkin, A. B. (2001). Engineering applications of noncommutitative harmonic analysis: with emphasis on rotation and motion groups. Boca Raton: CRC Press.

Citti, G., \& Sarti, A. (2006). A cortical based model of perceptional completion in the roto-translation space. Journal of Mathematical Imaging and Vision, 24(3), 307-326.

Descoteaux, M., Angelino, E., Fitzgibbons, S., \& Deriche, R. (2007). Regularized, fast, and robust analytical Q-ball imaging. Magnetic Resonance in Medicine, 58(3), 497-510.

Driscoll, J. R., \& Healy, D. M. (1994). Computing Fourier transforms and convolutions on the 2-sphere. Advances in Applied Mathematics, 15(2), 202-250.

Duits, R., \& Burgeth, B. (2007). Scale spaces on Lie groups. In M. Sgallari \& Paragios (Eds.), Scale space and variational methods, (Ischia, Italy) (pp. 300-312). Berlin: Springer.

Duits, R., \& Franken, E. M. (2009). Line enhancement and completion via left-invariant scale spaces on $S E(2)$. In Lecture notes in computer science: Vol. 5567. Proceedings 2nd international conference on scale space and variational methods in computer vision (pp. 795-807). Berlin: Springer.

Duits, R., \& Franken, E. M. (2009). Left-invariant diffusions on $\mathbb{R}^{3} \rtimes S^{2}$ and their application to crossing-preserving smoothing on HARDI images. CASA-report Department of Mathematics and Computer Science, Technische Universiteit Eindhoven. Nr. 18, May 2009. Available on the web http://www.win.tue.nl/casa/ research/casareports/2009.html.

Duits, R., \& Franken, E. M. (2010). Left invariant parabolic evolution equations on $S E(2)$ and contour enhancement via invertible orientation scores, part I: Linear left-invariant diffusion equations on SE(2), part II: Nonlinear left-invariant diffusion equations on invertible orientation scores. Quarterly on Applied Mathematics, 68(2), 255-331.

Duits, R., \& van Almsick, M. (2008). The explicit solutions of linear left-invariant second order stochastic evolution equations on the 2d-euclidean motion group. Quarterly of Applied Mathematics, American Mathematical Society, 66, 27-67.

Duits, R., Florack, L. M. J., de Graaf, J., \& ter Haar Romeny, B. M. (2004). On the axioms of scale space theory. Journal of Mathematical Imaging and Vision, 20, 267-298.

Duits, R., Felsberg, M., Granlund, G., \& ter Haar Romeny, B. M. (2007). Image analysis and reconstruction using a wavelet transform constructed from a reducible representation of the euclidean motion group. International Journal of Computer Vision, 72(1), 79-102.

Duits, R., Führ, H., \& Janssen, B. J. (2009). Left invariant evolutions on Gabor transforms. CASA-report Department of Mathematics and Computer Science, Technische Universiteit Eindhoven. Nr. 9, February 2009. Available on the web http://www.win.tue.nl/casa/ research/casareports/2009.html.

Fletcher, T., \& Joshi, S. (2007). Riemannian geometry for the statistical analysis of diffusion tensor data. Signal Processing, 87(2), 250 262.

Florack, L. M. J. (2008). Codomain scale space and regularization for high angular resolution diffusion imaging. In S.A. Fernández, \& R. de Luis Garcia (Eds.), Digital proceedings of CVPR workshop on tensors in image processing and computer vision, Anchorage, Alaska, USA, June 24-26, 2008. New York: IEEE.

Florack, L. M. J., \& Balmachnova (2008). Decomposition of high angular resolution diffusion images into a sum of self-similar polynomials on the sphere. In Proceedings of the eighteenth international conference on computer graphics and vision, GraphiCon'2008 (pp. 26-31). Russian Federation, Moscow. 
Franken, E. M. (2008). Enhancement of crossing elongated structures in images. $\mathrm{PhD}$ thesis, Eindhoven University of Technology, Department of Biomedical Engineering, The Netherlands.

Franken, E. M., \& Duits, R. (2009). Crossing preserving coherenceenhancing diffusion on invertible orientation scores. International Journal of Computer Vision, 85(3), 253-278.

Gaveau, B. (1977). Principe de moindre action, propagation de la chaleur et estimees sous elliptiques sur certains groupes nilpotents. Acta Mathematica, 139, 96-153.

Gerschgorin, S. (1931). Über die Abgrenzung der Eigenwerte einer Matrix. Izvestia Akademii Nauk USSR. Otdelenie Fiz.-Mat. Nauk, 7, 749-754.

Gur, Y., \& Sochen, N. (2005). Denoising tensors via Lie group flows. In Lecture notes in computer science: Vol. 3752. Variational, geometric, and level set methods in computer vision (pp. 195-208). Berlin: Springer.

Gur, Y., \& Sochen, N. (2009). Regularizing flows over Lie groups. Journal of Mathematical Imaging and Vision, 33(2), 195-208.

Hess, C. P., Mukherjee, P., Tan, E. T., Xu, D., \& Vigneron, D. B. (2006). Q-ball reconstruction of multimodal fiber orientations using the spherical harmonic basis. Magnetic Resonance in Medicine, 56, 104-117.

Hormander, L. (1968). Hypoelliptic second order differential equations. Acta Mathematica, 119, 147-171.

Iijima, T. (1962). Basic theory on normalization of a pattern (in case of typical one-dimensional pattern). Bulletin of Electrical Laboratory, 26, 368-388 (in Japanese).

Jost, J. (2005). Riemannian geometry and geometric analysis, 4th ed. Heidelberg: Springer.

Koenderink, J. J. (1984). The structure of images. Biological Cybernetics, 50, 363-370.

Kunis, S., \& Potts, D. (2003). Fast spherical Fourier algorithms. Journal of Computational and Applied Mathematics, 161(1), 75-98.

Mumford, D. (1994). Elastica and computer vision. In Algebraic geometry and its applications (pp. 491-506). Berlin: Springer.
Nagel, A., Ricci, F., \& Stein, E.M. (1990). Fundamental solutions and harmonic analysis on nilpotent groups. Bulletin of American Mathematical Society, 23, 139-144.

Özarslan, E., \& Mareci, T. H. (2003). Generalized diffusion tensor imaging and analytical relationships between diffusion tensor imaging and high angular resolution imaging. Magnetic Resonance in Medicine, 50, 955-965.

Prčkovska, V., Rodrigues, P., Duits, R., ter Haar Romeny, B. M., \& Vilanova, A. Extrapolating fiber crossings from DTI data. Can we gain the same information as HARDI? Technical CASA report $n r$. 20, 2010, Department of Mathematics and Computer Science, Eindhoven University of Technology, http://www.win.tue.nl/casa/research/casareports/2010.html.

Rodrigues, P., Duits, R., ter Haar Romeny, B. M., \& Vilanova, A. Accelerated diffusion operators for enhancing DW-MRI. Technical CASA report, nr. 15, 2010, Department of Mathematics and Computer Science, Eindhoven University of Technology, http://www.win.tue.nl/casa/research/casareports/2010.html.

Spivak, M. (1975). Differential geometry (Vol. 2). Berkeley: Publish or Perish.

ter Elst, A. F. M., \& Robinson, D. W. (1998). Weighted subcoercive operators on Lie groups. Journal of Functional Analysis, 157, 88163.

Thornber, K. K., \& Williams, L. R. (2000). Characterizing the distribution of complete shapes with corners using a mixture of random processes. Patern Recognition, 33, 543-553.

van Almsick, M. A. (2005). Context models of lines and contours. $\mathrm{PhD}$ thesis, Eindhoven University of Technology, Department of Biomedical Engineering, Eindhoven, The Netherlands. ISBN:97890-386-1117-4.

Weickert, J. A. (1999). Coherence-enhancing diffusion filtering. International Journal of Computer Vision, 31(2/3), 111-127.

Zweck, J., \& Williams, L.R. (2004). Euclidean group invariant computation of stochastic completion fields using shiftable-twistable functions. Journal of Mathematical Imaging and Vision, 21(2), $135-154$. 